University of Louisville

ThinkIR: The University of Louisville's Institutional Repository

Electronic Theses and Dissertations

$5-2021$

\title{
Identifying the cardiovascular effects of multiple pollutants.
}

\author{
Katlyn Elizabeth McGraw \\ University of Louisville
}

Follow this and additional works at: https://ir.library.louisville.edu/etd

Part of the Cardiology Commons, Chemical Actions and Uses Commons, Complex Mixtures

Commons, Environmental Health Commons, Environmental Public Health Commons, Inorganic Chemicals

Commons, and the Organic Chemicals Commons

\section{Recommended Citation}

McGraw, Katlyn Elizabeth, "Identifying the cardiovascular effects of multiple pollutants." (2021). Electronic Theses and Dissertations. Paper 3605.

https://doi.org/10.18297/etd/3605

This Doctoral Dissertation is brought to you for free and open access by ThinkIR: The University of Louisville's Institutional Repository. It has been accepted for inclusion in Electronic Theses and Dissertations by an authorized administrator of ThinkIR: The University of Louisville's Institutional Repository. This title appears here courtesy of the author, who has retained all other copyrights. For more information, please contact thinkir@louisville.edu. 
IDENTIFYING THE CARDIOVASCULAR EFFECTS OF MULTIPLE POLLUTANTS

By

Katlyn Elizabeth McGraw

B.A., University of Louisville, 2009

M.P.H. University of Louisville, 2016

A Dissertation

Submitted to the Faculty of the

School of Public Health and Information Sciences

In Partial Fulfillment of the Requirements for the Degree of

Doctor of Philosophy

in Public Health Sciences

Department of Environmental and Occupational Health

Sciences University of Louisville

Louisville, KY

May 2021 
Copyright 2021 by Katlyn Elizabeth McGraw

All Rights Reserved 

IDENTIFYING THE CARDIOVASCULAR EFFECTS OF MULTIPLE POLLUTANTS

\author{
By \\ Katlyn Elizabeth McGraw \\ B.A., University of Louisville, 2009 \\ M.P.H. University of Louisville, 2016 \\ A Dissertation Approved on \\ April 7th, 2021 \\ By the following Dissertation Committee
}

Dissertation Chair, Aruni Bhatnagar

Dissertation Co-Chair, Gary Hoyle

Kira Taylor

Qunwei Zhang

Shesh N. Rai

Sanjay Srivastava 


\section{DEDICATION}

This dissertation is dedicated to my parents

Dr. Charles Patrick McGraw

and

Ms. Janice McGraw

to whom I am forever grateful

for teaching me the value of education and the importance of challenging yourself. 


\section{ACKNOWLEDGEMENTS}

I would like to thank my mentor, Dr. Aruni Bhatnagar, for his guidance and his passion for evidence-based research. I would also like to thank my committee members and my advisor, Dr. Gary Hoyle, for supporting me in this endeavor and providing feedback. To my partner, Victoria, thank you for your love, support, and consistent belief in me. Additionally, I want to acknowledge my dissertation companion and dear friend, Arbor Quist, for being a light during many tough times, even from many states away. I want to acknowledge the Accountabilibuddies group who helped structure my time to write this dissertation. Also, I want to give many thanks to my family and friends who have always been excited about my work and proud of my achievements. 


\section{ABSTRACT \\ IDENTIFYING THE CARDIOVASCULAR EFFECTS OF MULTIPLE POLLUTANTS \\ Katlyn E. McGraw \\ 7 April 2021}

Cardiovascular disease (CVD) is the leading cause of death from environmental exposures. Although exposure to $\mathrm{PM}_{2.5}$ is an established risk factor for CVD, the contribution of other hazardous pollutant exposure to CVD is less clear. Overall, this work aimed to examine the effect of pollutants with lesser documented effects on cardiovascular disease using a multipronged approach to exposure assessment. The three aims were to examine the relationship between county-level toxic chemical releases and CVD mortality in the contiguous United States between 2002 and 2012, to assess the relationship between individual-level VOC metabolites and vascular function, and to build multipollutant models from the previous two aims to assess the role of mixtures and mixture components in CVD mortality and vascular function. In our national, county-level study, we found that for every $25 \%$ increase in annual county-level toxic release, we found a $2.8 \%(1.2,4.4 ; p$-value $=0.0006)$ increase in CVD mortality rate. We also found that for every $25 \%$ increase in annual county-level risk score, there was a $3.0 \%(95 \% \mathrm{Cl}$ 1.3, 4.6; $p$-value $=0.0003$ ) increase in CVD mortality. Using the multipollutant method, elastic net, we identified five out of 467 potentially toxic chemicals at the county-level: bromoform, dichlorobromomethane, dichlorotrifluoroethane, nitrophenol, and thallium. In our study of individual-level VOC metabolites, we found that the acrolein metabolite, 3HPMA, was positively associated with systolic BP (+0.98 per SD of 3HPMA; Cl: $0.04,1.91 ; \mathrm{P}=0.04)$. For each IQR of 3HPMA or DHBMA (a 1,3-butadiene metabolite), there was a $3.3 \%(\mathrm{Cl}:-6.18,-0.37$; p-value: $0.03)$ or a $4.0 \%(\mathrm{Cl}:-7.72,-0.12 ; \mathrm{P}=0.04)$ decrease in endothelial function. Urinary levels of the 1,3-butadiene metabolite, MHBMA3, were positively associated with a $2.9 \%$ increase in urinary epinephrine ( $\mathrm{Cl}: 0.48,5.37 ; \mathrm{P}=0.02)$. Using the multipollutant method, Bayesian Kernel Machine 
Regression, we found that the whole mixture of VOC metabolites (CEMA, 3HPMA, DHBMA, MHBMA3, and HPMMA) was significantly associated with blood pressure, which was primarily driven by 3HPMA. Taken together, these findings suggest that exposure to under regulated pollutants like TRI chemicals and VOCs contribute to CVD mortality and vascular dysfunction. Further research is required to corroborate these findings. 
TABLE OF CONTENTS

DEDICATION

ACKNOWLEDGEMENTS $\quad$ iv

ABSTRACT

LIST OF TABLES $\quad X$

LIST OF FIGURES

CHAPTER 1 POLLUTION AND CARDIOVASCULAR DISEASE

INTRODUCTION

BACKGROUND 3

General Overview of VOCs 3

$\begin{array}{ll}\text { Regulation of VOCs } & 7\end{array}$

Surveillance of VOCs 9

Measurement of VOCs in Air 13

Risk Assessment Framework $\quad 15$

Conceptual Framework 18

Literature Review of Relationship between VOC Exposure and CVD 19

PROBLEM STATEMENT 26

PURPOSE OF THE STUDY $\quad 26$

RESEARCH QUESTIONS AND HYPOTHESES

RATIONALE, RELEVANCE, AND SIGNIFICANCE OF THE STUDY 28

SUMMARY AND ORGANIZATION OF THE REMAINDER OF THE STUDY 31

DEFINITION OF TERMS 32

CHAPTER 2 COUNTY LEVEL TOXIC RELEASES ARE ASSOCIATED WITH

CARDIOVASCULAR MORTALITY BETWEEN 2002 AND 2012

INTRODUCTION

METHODS 36

Study Design and Population 36 
Data Sources $\quad 37$

Units of Analysis $\quad 39$

Missing Data $\quad 41$

Data Distribution $\quad 42$

Statistical Analyses $\quad 43$

Model Fitting $\quad 44$

Key assumptions and limitations of the methods $\quad 45$

RESULTS $\quad 45$

DISCUSSION 51

CHAPTER 3 EXPOSURE TO VOLATILE ORGANIC COMPOUNDS IS ASSOCIATED WITH VASCULAR DYSFUNCTION

INTRODUCTION 54

METHODS 55

Measures of Vascular Function 56

Urinary Catecholamines and Metabolites 56

Urinary VOC Metabolites

Covariates $\quad 59$

Statistical Analysis $\quad 59$

Sensitivity Analysis $\quad 61$

RESULTS $\quad 62$

DISCUSSION 69

CHAPTER 4 POLLUTION MIXTURES ARE ASSOCIATED WITH CARDIOVASCULAR MORTALITY AND BLOOD PRESSURE

$\begin{array}{ll}\text { INTRODUCTION } & 75\end{array}$

$\begin{array}{ll}\text { METHODS } & 76\end{array}$

Study Populations, Exposures, and Outcomes $\quad 76$

National County Level TRI Study 76

Louisville Healthy Heart Study $\quad 77$

$\begin{array}{ll}\text { Statistical Analysis } & 79\end{array}$

$\begin{array}{ll}\text { Variable Selection } & 79\end{array}$

Bayesian Kernel Machine Regression $\quad 81$

RESULTS $\quad 82$

Variable Selection $\quad 82$ 
Bayesian Kernel Machine Regression $\quad 85$

DISCUSSION 88

CHAPTER 5 SUMMARY AND CONCLUSIONS 92

SUMMARY OF STUDY $\quad 92$

MAJOR FINDINGS $\quad 92$

National County Level TRI Study 92

Louisville Healthy Heart Study 93

Mixture Assessment 94

STRENGTHS $\quad 95$

LIMITATIONS $\quad 96$

FUTURE DIRECTIONS 98

CONCLUSIONS 99

$\begin{array}{ll}\text { REFERENCES } & 100\end{array}$

APPENDICES

APPENDIX A 111

CURRICULUM VITAE $\quad 122$ 


\section{LIST OF TABLES}

Table 1-1. Hazardous Air Pollutants under the Clean Air Act..................................................... 6

Table 1-2. Toxic organic (TO) method for quantifying toxic organic compounds in air as defined

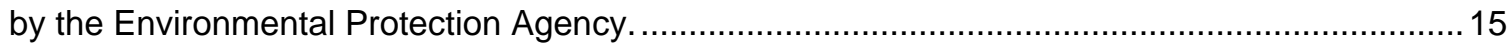

Table 1-3. Volatile organic compound parent names, urinary metabolite long names, short names, and estimated half-lives.

Table 2-1. Descriptive statistics (number and median [minimum, maximum]) for counties in the contiguous U.S. for the baseline year, 2002 and the final year, 2012

Table 3-1. Descriptive Statistics [number (\%) and mean ( \pm standard deviation (SD))] for nonsmoking participants in the Louisville Heathy Heart Study ( $n=346)$, stratified by low and high VOC metabolite concentrations.

Table 4-1. Descriptive statistics [mean ( \pm standard deviation (SD))] for county-level characteristics in the National County Level Study $(n=2,287)$ in 2012 .

Table 4-2. Mean squared errors (MSE) and tuning parameters of the three variable selection techniques, ridge regression, lasso, and elastic net. The ridge regression uses a tuning parameter of $\alpha=0$, lasso uses a tuning parameter of $\alpha=1$, and the elastic net procedure uses a tuning parameter of $\alpha=0.3$.

Table 4-3. Posterior inclusion probabilities of individual VOC metabolites in the Bayesian Kernel Machine Regression model. 


\section{LIST OF FIGURES}

Figure 1-1. Federal air monitoring programs monitoring hazardous air pollutants in the contiguous United States.

Figure 1-2. Select air monitoring stations measuring volatile organic compounds classified as hazardous air pollutants in the contiguous United States.

Figure 1-3. Cost and exposure approximation tradeoffs in exposure assessment methods. 17

Figure 2-1. Data acquisition flow chart for the National County Level TRI Study..... 42

Figure 2-2. Toxic Release Inventory (TRI) sites in dark blue and hazardous waste sites (NPL) in light blue in the contiguous United States.

Figure 2-3. Secular trends in county-level TRI releases and All Cause and CVD Cause Mortality between 2002 and 2012

Figure 2-4. Top chemicals released at the county-level, annually, between 2002 and 2012 in the contiguous United States.

Figure 2-5. Comparison of county-level exposure indices, county sum and risk sum, in the contiguous United States. .50

Figure 2-6. Comparison of counties with highest annual county sum and risk sum. 50

Figure 2-7. Percent increase in annual, county-level mortality rates (per $100 \mathrm{k}$ people) per $25 \%$ increase in county sum or risk sum.

Figure 3-1. Exposure distributions of each VOC metabolite, CEMA, 3HPMA, DHBMA, MHBMA3, and HPMMA, reported as totals $(A)$, and stratified by sex $(B)$ and race $(C)$. 66 Figure 3-2. Differences ( $\mathrm{mmHg}$ ) and percent differences, and 95\% confidence intervals in blood pressure and RHI per IQR of VOC metabolite (A). Predicted means and 95\% confidence intervals $(\mathrm{mmHg})$ in blood pressure and \% difference in $\mathrm{RHI}$ with increasing tertile of 3HPMA and 
DHBMA (B). Differences and 95\% confidence intervals $(\mathrm{mmHg})$ in blood pressure, stratified by sex and race $(\mathrm{C})$

Figure 3-3. Percent differences and 95\% confidence intervals in catecholamines per IQR of VOC metabolite in the total cohort $(+)$. Models were stratified by male participants (filled circle), female participants (open circle), Black participants (fliled triangle), and White participants (open triangle)

Figure 4-1. Beta coefficients plotted for each selected variable in the elastic net regularization technique

Figure 4-2. Beta coefficients plotted for each selected variable in the elastic net regularization technique.

Figure 4-3. Single-VOC metabolite exposure response association when all other metabolites are fixed to their 50th percentile... .86

Figure 4-4. Overall effect of the VOC mixture on systolic and diastolic blood pressure when all other predictors are at a particular percentile when compared with the value when all predictors are at their $50^{\text {th }}$ percentile.

Figure 4-5. Systolic and diastolic blood pressure for an interquartile range increase comparison of individual VOC metabolite concentrations when all other VOC metabolites have been fixed to the 25th, 50th, and 75th percentiles. 


\section{CHAPTER 1 POLLUTION AND CARDIOVASCULAR DISEASE}

\section{INTRODUCTION}

Cardiovascular disease (CVD) is the leading cause of death from environmental exposures, surpassing cancer mortality. ${ }^{1}$ In 2017, the Lancet Commission on Pollution and Health estimated the global burden of disease caused by pollution is responsible for 9 million premature deaths annually. ${ }^{2}$ Approximately seven million premature deaths are attributed to either household or ambient air pollution. ${ }^{3}$ The World Health Organization estimates that exposure to air pollution is associated with 1.4 million deaths from stroke and 2.4 million deaths from heart disease, annually. ${ }^{3}$

Air pollution is a mixture of particulates and gaseous components, including particulate matter, nitrogen oxides, sulfur dioxides, ozone, carbon monoxide, organic and black carbon, and volatile organic compounds (VOCs).The majority of air pollution studies use population weighted, annual mean concentrations of particulate matter of diameter $\leq 2.5 \mu \mathrm{m}\left(\mathrm{PM}_{2.5}\right)$ and tropospheric ozone $\left(\mathrm{O}_{3}\right)$ to estimate air pollution exposure-related mortality. ${ }^{4}$ However, even though the links between exposure to these two pollutants and CVD are well-documented, ${ }^{5-7} \mathrm{PM}_{2.5}$ and $\mathrm{O}_{3}$ only represent a portion of multifactorial pollution. Therefore, there is a need to understand the role of other pollutants in the development of CVD in exposed individuals.

Volatile organic compounds (VOCs) are gaseous compounds that comprise a major part of indoor and outdoor air pollution. Primary sources of VOCs are combustion from tobacco smoke and vehicle exhaust, industrial releases, hazardous waste sites, consumer goods production, and products that contain organic solvents. ${ }^{8}$ VOCs also interact with other pollutants to create intermediates and secondary pollutants such as tropospheric $\mathrm{O}_{3}$ and secondary organic aerosols (SOAs), a major component of particulate matter. ${ }^{9,} 10$ However, compared with PM2.5, VOC

exposure approximation is complicated by a lack of routine air monitoring of VOCs, ${ }^{11,}{ }^{12}$ large 
variations of VOCs in space and time, ${ }^{13,14}$ and a high volume of chemicals in production each year. ${ }^{15}$ Despite decreasing national trends in pollutants, ${ }^{11,12,16}$ VOCs remain under-regulated and uncharacterized in relation to cardiovascular health.

Although there are difficulties inherent in the quantification of VOCs and VOC exposure, some evidence suggests exposure to VOCs causes adverse health effects such as CVD. Previous epidemiological studies have shown significant associations between exposure to ambient VOCs such as alkenes, alkynes, and BTEX compounds (benzene, toluene, ethyl benzene and xylene) and cardiovascular events such as heart failure, ${ }^{17}$ stroke, ${ }^{18}$ ischemic heart disease, ${ }^{18,}{ }^{19}$ and cardiovascular mortality rates. ${ }^{20}$ Indoor exposure to total VOCs has been associated with increased blood pressure and heart rate, ${ }^{21}$ and VOC metabolites measured in blood are associated with an increased prevalence of doctor-diagnosed CVD in the National Health and Nutrition Examination Survey (NHANES) cohort. ${ }^{22}$ Hazardous air pollutants (HAPs) released from industrial facilities are associated with cardiovascular mortality in an ecological study, many of which are classified as VOC. ${ }^{19}$ Likewise, VOC-containing mixtures of pollutants such as automobile and diesel engine emissions, and tobacco smoke have been linked to the development of CVD. ${ }^{23-29}$ However, the role of individual VOCs and mixtures of VOCs in CVD pathophysiology remains to be identified in human studies.

To better understand the role of VOC exposure in the development of CVD, I proposed three distinct methods to assess human exposure to VOCs: source proximity, human biomonitoring, and mixture modelling. These three methods were then used to assess the relationship between VOC exposure and CVD, the overall research goal. This study fills a gap in existing literature around VOCs, specifically in exposure assessment methods, as well as presenting further evidence of a relationship between VOC exposure and CVD. Although these exposure assessment methods have been used before, this study extends prior research by using both aggregate and individual level data, longitudinal data, mixed models, and mixture analyses to improve previously published work. This study improves and provides new models to elucidate the link between exposure to VOCs and CVD in humans. 
To provide context for this study, I briefly describe national VOC trends, classification, and sources of VOCs. Next, I describe the regulation, surveillance, and measurement of VOCs to give context to the exposure assessment problem. I then evaluate the literature on exposure to VOCs and CVD in ecological and epidemiological studies. The problem is then stated with an outline of the hypotheses and aims. I further introduce the rationale, relevance, and significance of the study, and describe assumptions, limitations, and delimitations of the study. Terms are defined and a description of the following chapters ends the introductory chapter.

\section{BACKGROUND}

\section{General Overview of VOCs}

The National Emissions Inventory Report estimated VOC emissions represent $13.7 \%$ of total emissions in 2014. Despite reductions in VOC emissions from approximately 20.2 million tons in 2002 to 16.9 million tons in $2014,{ }^{30}$ VOCs continue to be prevalent as they arise from other major, area, and mobile sources. The regulation of VOCs occurs largely via maximum control technologies, emission reduction programs, and mobile source regulation. However, the extent to which VOCs are present in our environment is underestimated by the lack of routine monitoring. Thus, it is important to acknowledge the impact of VOCs in our environment and to better understand their role in contributing to adverse health effects.

VOCs represent a large class of chemicals defined by high vapor pressure, low boiling point, and low molecular weight. Therefore, these compounds volatilize quickly into the atmosphere, signifying their importance as an environmental pollutant in indoor and outdoor air. They contain carbon and are emitted from liquids and solids, or are released during combustion. ${ }^{31}$ High vapor pressure is the property which first led to regulation and nomenclature of VOCs due to their ability to vaporize into the atmosphere and contribute to photochemical smog or ozone, a

federally regulated secondary pollutant. The U.S. Environmental Protection Agency (EPA) defines a VOC as '....any compound of carbon, excluding carbon monoxide, carbon dioxide, carbonic acid, metallic carbides or carbonates, and ammonium carbonate, which participates in atmospheric photochemical reactions. ${ }^{32}$ Although VOCs are prevalent in air as primary and 
secondary pollutants, they also contaminate soil and drinking water, especially in close proximity to hazardous waste sites. ${ }^{33,34}$

VOCs are ubiquitous in the environment, varying spatially and temporally because of weight and reactivity. ${ }^{20}$ They arise from both anthropogenic and natural sources. Natural sources include volcanoes, vegetation, and animals. Anthropogenic sources include combustion processes such as vehicle exhaust or fuel combustion, industry emissions, chemical feedstocks to produce consumer products, oil and gas, and waste sites. Source types include point, area, and mobile sources. Point sources, or major sources, are stationary sources such as industrial facilities, chemical plants, steel mills, oil refineries and hazardous waste incinerators. Area sources are multiple, smaller sources which release pollutants into the air like dry cleaners, gas stations, and woodstoves. Mobile sources are cars, trucks, trains, airplanes, marine vessels, and other non-road equipment. Major sources are divided into sectors: agriculture, dust, fire, fuel combustion, industrial processes, miscellaneous, mobile, and solvent. Point source pollution is a localized source of toxics while area source emissions are well-distributed. Federal regulation of VOCs occurs only at area and point sources.

VOCs are a major source of indoor pollution as well. The average person spends at least $80 \%$ of time indoors. ${ }^{35}$ According to the EPA, indoor VOC levels are much higher than outdoor, mostly due to inadequate ventilation after diffusion of ambient pollution indoor. Indoor VOCs come from many household and common consumer products such as cosmetics, personal care products, furniture, cleaning products, and building materials. Thus, these organics are ubiquitous in the indoor and outdoor environment, threatening all routes of human exposure.

Exposure to VOCs can cause varied human health effects, such as neurological disorders, organ toxicity, and cancer, dependent on the chemical compound. Many VOCs are thereby classified hazardous air pollutants (HAPs) or air toxics. ${ }^{36}$ The Clean Air Act (CAA) lists 188 chemicals as HAPs known to be harmful to human health (Table 1-1), several of which are VOCs. Federal public health and regulatory agencies, such as the Environmental Protection Agency (EPA), the Agency for Toxic Substances and Disease Registry (ATSDR), and the Occupational Health and Safety Administration (OSHA) publish permissible rates of human 
exposure, based on established toxicological studies of humans and animals. However, many permissible rates originate from occupational cohorts with large doses, values not indicative of 
Table 1-1.

Hazardous Air

Pollutants under the

Clean Air Act.

Acetaldehyde

Acetamide

Acetonitrile

Acetophenone

2-Acetylaminofluorene

Acrolein

Acrylamide

Acrylic acid

Acrylonitrile

Allyl chloride

4-Aminobiphenyl

Aniline

o-Anisidine

Asbestos

Benzene-including

gasoline

Benzidine

Benzotrichloride

Benzyl chloride

Biphenyl

Bis(2-ethylhexyl)phthalate

Bis(chloromethyl)ethe

Bromoform

1,3-Butadiene

Calcium cyanamide

Caprolactam

Captan

Carbary

Carbon disulfide

Carbon tetrachloride

Carbonyl sulfide

Catechol
Chloramben

Chlordane

Chlorine

Chloroacetic acid

2-Chloroacetophenone

Chlorobenzene

Chlorobenzilate

Chloromethyl methyl ether

Chloroprene

Cresols/Cresylic acid

o-Cresol

m-Cresol

p-Creso

Cumene

2,4-D, salts and esters

DDE

Diazomethane

Dibenzofurans

1,2-Dibromo-3-

chloropropane

Dibutylphthalate

1,4-Dichlorobenzene $(p)$

3,3-Dichlorobenzidene

Dichloroethyl ether

1,3-Dichloropropene

Dichlorvos

Diethanolamine

$\mathrm{N}, \mathrm{N}$-Dimethylaniline

Diethyl sulfate

3,3-Dimethoxybenzidine

Dimethyl

aminoazobenzene

3,3'-Dimethyl benzidine

Dimethyl carbamoy

chloride

Dimethyl formamide

1,1-Dimethyl hydrazine

Dimethyl phthalate

Dimethyl sulfate

4,6-Dinitro-o-creso

2,4-Dinitrophenol

2,4-Dinitrotoluene
1,4-Dioxane

1,2-Diphenylhydrazine

Epichlorohydrin

1,2-Epoxybutane

Ethyl acrylate

Ethyl benzene

Ethyl carbamate

Ethylene dibromide

Ethylene dichloride

Ethylene glyco

Ethylene imine

Ethylene oxide

Ethylene thiourea

Ethylidene dichloride

Formaldehyde

Heptachlor

Hexachlorobenzene

Hexachlorobutadiene

Hexachlorocyclopentadie

Hexachloroethane

Hexamethylene-1,6-

diisocyanate

Hexamethylphosphoramid

Hexane

Hydrazine

Hydrochloric acid

Hydrogen fluoride

Hydrogen sulfide

Hydroquinone

Isophorone

Lindane

Maleic anhydride

Methanol

Methoxychlor

Methyl bromide

Methyl chloride

Methyl chloroform

Methyl ethyl ketone

Methyl hydrazine

Methyl iodide

Methyl isobutyl ketone

Methyl isocyanate
Methyl methacrylate

Methyl tert butyl ether

4,4-Methylene bis(2

chloroaniline)

Methylene chloride

Methylene diphenyl

diisocyanate (MDI)

4,4'-Methylenedianiline

Naphthalene

Nitrobenzene

4-Nitrobipheny

4-Nitrophenol

2-Nitropropane

$\mathrm{N}$-Nitroso-N-methylurea

$\mathrm{N}$-Nitrosodimethylamine

$\mathrm{N}$-Nitrosomorpholine

Parathion

Pentachloronitrobenzene

Pentachlorophenol

Phenol

p-Phenylenediamine

Phosgene

Phosphine

Phosphorus

Phthalic anhydride

Polychlorinated biphenyls

1,3-Propane sultone

beta-Propiolactone

Propionaldehyde

Propoxur

Propylene dichloride

Propylene oxide

1,2-Propylenimine

Quinoline

Quinone

Styrene

Styrene oxide

2,3,7,8-

Tetrachlorodibenzo-p-

dioxin

$1,1,2,2-$

Tetrachloroethane

Tetrachloroethylene
Titanium tetrachloride

Toluene

2,4-Toluene diamine

2,4-Toluene diisocyanate

o-Toluidine

Toxaphene

1,2,4-Trichlorobenzene

1,1,2-Trichloroethane

Trichloroethylene

2,4,5-Trichlorophenol

2,4,6-Trichlorophenol

Triethylamine

Trifluralin

2,2,4-Trimethylpentane

Vinyl acetate

Vinyl bromide

Vinyl chloride

Vinylidene chloride

Xylenes all isomers

o-Xylenes

p-Xylenes

Antimony Compounds

Arsenic Compounds

Beryllium Compounds

Cadmium Compounds

Chromium Compounds

Cobalt Compounds

Coke Oven Emissions

Cyanide Compounds

Glycol ethers

Lead Compounds

Manganese Compounds

Mercury Compounds

Fine mineral fibers

Nickel Compounds

Polycyclic Organic Matter

Radionuclides

Selenium Compounds

Abundance of data: > 20 monitoring sites with sufficient data to create a valid annual average between 2003-2005, up to 434 sites

Little data: < 20 monitoring sites with sufficient data to create a valid annual average between 2003-2005, between 1-17 sites

No data: No valid annual averages between 2003-2005 from http://www.epa.gov/ttn/atw/188polls.html 
the public health impact. Furthermore, toxicological studies focus on organ toxicity and more easily measurable effects such as cancer, in lieu of more prevalent chronic diseases, such as CVDs. Therefore, the effect of VOC exposure on cardiovascular and metabolic toxicity remains under characterized.

\section{Regulation of VOCs}

Due to the ubiquity and harmful effects of VOCs, the federal government promulgates regulations for emission reduction and efforts to reduce adverse health effects. There are two major federal laws governing VOCs, the Clean Air Act (CAA), and the Emergency and Community Right to Know Act (EPCRA). Both laws are part of Title 42 of the United States Code on Public Health and Welfare. Title 40 is the Protection of the Environment which largely outlines how these promulgations will be followed. The CAA was first promulgated in 1970 and continues to be amended to meet public health and welfare needs. In Title 42, Chapter 85, Subchapter 1 Part $A$ of the U.S. code, congress recognizes that rapid urbanization, growth in amount and complexity of air pollution, and ability of pollution to cross boundaries as important evidence for the regulation of air pollution for the protection of public health and welfare. The U.S. code specifically declares 'to protect and enhance the quality of air resources to promote public welfare and productive capacity of the working public. ${ }^{37}$

The key regulations of the CAA are the primary and secondary national ambient air quality standards (NAAQS). Six criteria pollutants were named NAAQS: particulate matter (PM), ozone $\left(\mathrm{O}_{3}\right)$, carbon monoxide $(\mathrm{CO})$, sulfur dioxide $\left(\mathrm{SO}_{2}\right)$, nitrogen dioxide $\left(\mathrm{NO}_{2}\right)$, and lead $(\mathrm{Pb})$. Established NAAQS are to be thoroughly reviewed by the administrator and an independent scientific review committee every five years to uphold applicability and relevance. States are required to adopt and submit a plan for implementation, maintenance, and enforcement of standards in each air quality region within the state. ${ }^{38}$ Conversely, VOCs do not have regulatory ambient air concentrations to meet in the CAA. Instead, Section 7412 of the CAA classifies a list of 188 hazardous air pollutants (HAPS) or air toxics, of which several are volatile organic pollutants. According to the section, emission regulations will be promulgated by source type: major, stationary, or area source; excluding mobile or off-road sources. Major sources are 
stationary or multiple stationary sources with potential to emit 10 tons per year of any HAP, or potential to emit 25 tons per year of any combination of HAPs. Hazardous air pollutants are any pollutant listed in section 7412 (and Table 1) and represent pollutants which present or may present adverse health effects including but not limited to carcinogenicity, teratogenicity, mutagenicity, neurotoxicity, reproductive dysfunction, or are acutely or chronically toxic through route of inhalation. HAPs also represent pollutants which present or may present adverse environmental effects whether through ambient concentrations, bioaccumulation, deposition, or releases.

Under the CAA, EPA must regulate HAPS using maximum allowable control technologies (MACTs) or national standards for fuels, cars, and trucks. Major sources releasing HAPS (10 tons per year) are regulated by standards requiring the application of air pollution controls known as MACTs. Air toxics or HAPS are monitored through an air toxics program which focuses primarily on reducing HAPS by applying available control technologies for industrial sources and area sources. Air toxics monitoring programs measure success in reduction in emissions, not air quality. The three primary objectives of the air toxics monitoring program are 1) determine trends and account for program progress, 2) support exposure assessments by providing comparison data for personal measurements, and 3) provide a basic structure for models for exposure assessments, emission control standards, and program effectiveness assessments. ${ }^{39}$ The National Air Toxics Monitoring Program uses three distinct monitoring methods: National Air Toxics Trends Stations, Community Scale projects, and existing state and local program monitoring. The EPA uses air dispersion modeling to estimate the impact of HAP emissions on ambient air and human health through the National Air Toxics Assessment.

The second major federal law governing VOCs in the environment is the Emergency Planning and Community Right to Know Act (EPCRA) which was first promulgated in 1986 as Title III of the Superfund Amendments and Reauthorization Act. Title 42, Chapter 116, Subchapters I, II, and III cover EPCRA. The chapter promulgates emergency planning and notification, reporting requirements, and general provisions regarding hazardous chemicals and extremely hazardous chemicals. Extremely hazardous substances require creation and 
submission of a comprehensive emergency response plan by the local emergency planning committee (LEPC). Alternatively, the owner or operator of a facility with ten or more employees that manufactures, processes, or otherwise uses more than 25,000 pounds of a hazardous chemical must complete a toxic chemical release form. The administrator of the EPA establishes and maintains a national toxic chemical release inventory based on data submitted under section Title 42 Chapter 116 Subchapter II Section 11023. The inventory is known as the annual toxic release inventory $(\mathrm{TRI})$.

\section{Surveillance of VOCs}

To monitor and regulate VOCs and air pollution, the National Emissions Inventory (NEI) program measures criteria pollutants, criteria precursors, and HAPs or air toxics from air emissions sources. National, regional, state, and local air monitoring is key to regulation and human health and ecology assessment. Ambient air monitoring is the systematic, long-term assessment of pollution in surrounding, outdoor air and is integral to managing and maintaining air quality and protecting public health. Some reasons to collect air pollution data are to assess the extent of pollution, provide air pollution data to the public, support implementation of air quality standards or goals, evaluate the effectiveness of emission control strategies, provide information on air quality trends, provide data for the evaluation of air quality models, and to support research. Monitoring plans are developed based on the type of pollutant and what the data is trying to achieve. ${ }^{40}$

Public health surveillance systematically collects data on specific health events affecting a population, analyzes and interprets those data, and effectively communicates those data to public health professionals and lawmakers. ${ }^{41}$ Air monitoring surveillance acquires data via stationary monitoring stations to meet four objectives: 1) to define air quality in heavily polluted areas, 2) to define air quality in less populated areas, 3) to provide area wide representation of ambient air quality, and 4) to define air quality with respect to the source category, or source, or both, to provide feedback relative to the effectiveness of adopted control strategies. ${ }^{42}$ National level surveillance helps to provide a clear distribution of the data while states collecting data 
provide more specific characteristics on a site by site basis. Monitoring is required at all levels to understand and reduce risk, the primary objective of air toxics monitoring. ${ }^{43}$

There are two major national monitoring efforts specific to monitoring air toxics: the National Air Toxics Trends Stations and the Urban Air Toxics Monitoring Program. Other ambient air monitoring programs developed for other purposes but which still provide air toxics data are Photochemical Assessment Monitoring Station program, the Chemical Speciation Network and the Speciation Trends Network, and the Interagency Monitoring of Protected Visual Environments (Figure 1-1 and 1-2). State and local government operate air toxics monitoring programs and design special studies to understand air toxics in their communities.

The National Air Toxics Trends Stations (NATTS) were first created in 2003 to meet the requirements of the Clean Air Act regarding the 187 HAPs causing adverse health effects. The primary objective of NATTS are to provide long-term monitoring of air toxics to assess trends, emission reduction program efficiency, and to assess and verify air quality models across the nation. The goal of the monitoring network is to be able to detect a $15 \%$ difference between successive 3-year annual mean concentrations. ${ }^{44}$ The NATTS currently include 27 national air toxics trends sites with 20 in urban areas and 7 in rural areas. The National Air Toxics Assessment (NATA) is a screening tool based on air quality monitoring and includes data from NATTS. NATA is a screening tool for state, local, and tribal agencies that assesses outdoor air quality with respect to emissions. NATA helps to identify areas of concern, characterize risk, and track progress. ${ }^{43}$ Air toxic concentrations are reported at the census level. Six assessments have been conducted and published for years 1996, 1999, 2002, 2005, 2011, and 2014.

The Urban Air Toxics Monitoring Program (UATMP) is a voluntary monitoring program which encourages state, local, and tribal agencies to understand the nature and extent of potentially toxic pollution in urban areas. The UATMP includes some NATTS, local scale monitoring stations, and approximately 105 funded air toxics monitoring sites. ${ }^{45}$ The Integrated Urban Air Toxics Strategy is part of the National Air Toxics Program and was developed by the EPA in 1999 to further regulate air toxics in urban areas by collectively looking at large and small industrial and commercial operations and mobile sources. The strategy addresses air toxics in 
Figure 1-1. Federal air monitoring programs monitoring hazardous air pollutants in the contiguous United States.

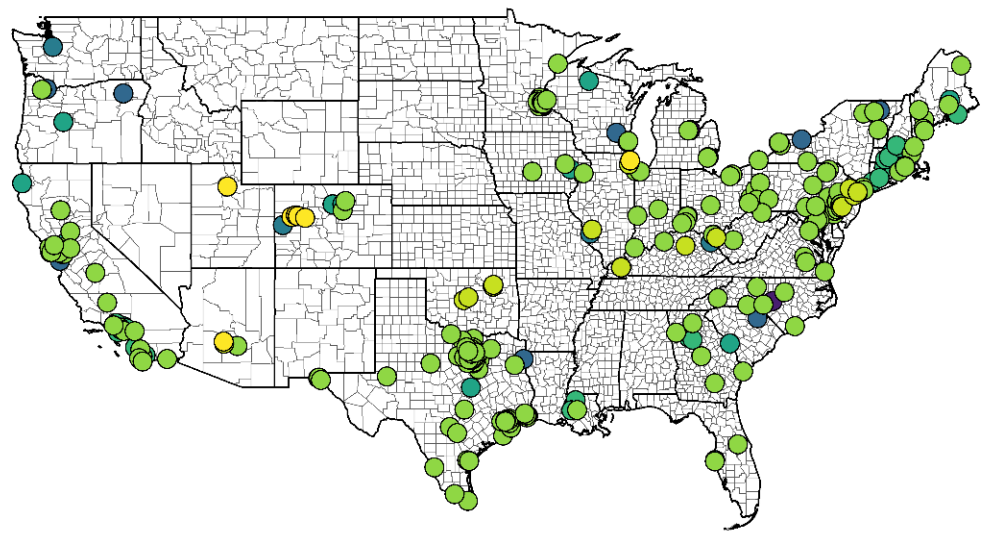

Monitoring Program

- industrial

LEE COUNTY BASELINE OIL GAS

- NATTS MQO CORE HAP: NON-UATMP

NATTS MQO CORE HAP: UATMP

NATTS NON-MQO CORE HAP: NON-UATMP

- NATTS NON-MQO CORE HAP: UATMP

NEAR ROAD

- NOAA GLOBAL MONITORING DIVISION

PAMS

PHILADELPHIA PS NETWORK

SLAMS

UATMP

UATMP: SNMOC

Figure 1-2. Select air monitoring stations measuring volatile organic compounds classified as hazardous air pollutants in the contiguous United States.

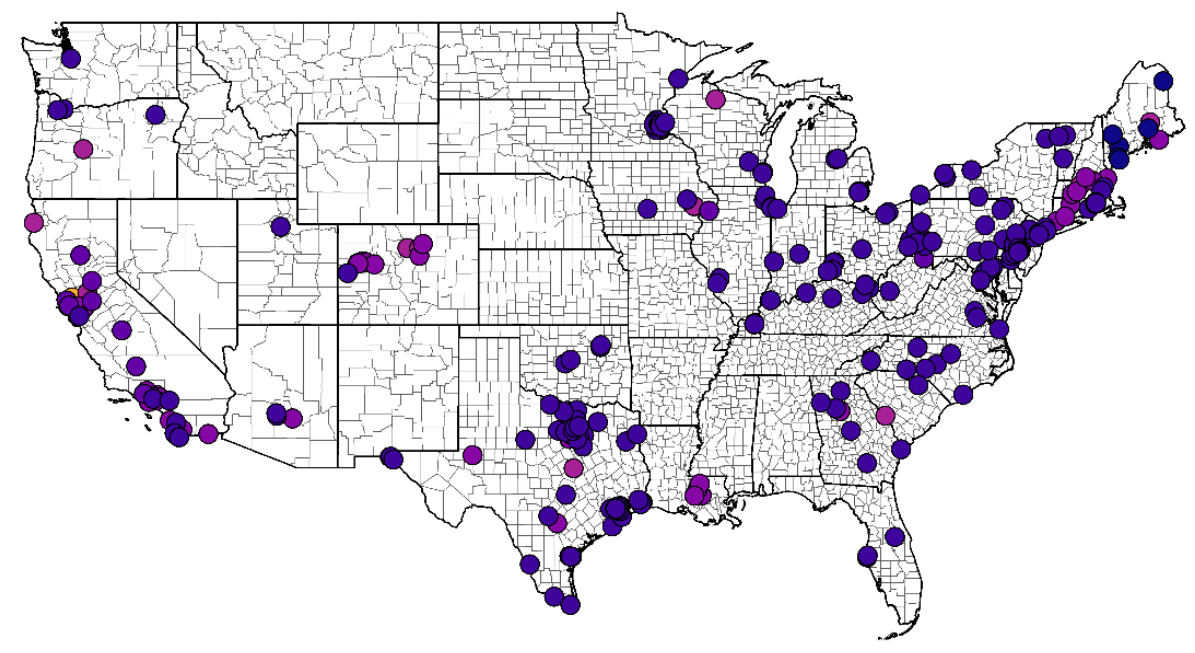


urban areas and outlines actions to reduce emissions and improve understanding of the health risks of air toxics. The goals of the strategy are 1) reduce by $75 \%$ the risk of cancer associated with air toxics from industrial and commercial sources; 2) substantially reduce noncancer health risks associated with air toxics from industrial and commercial sources; and 3) address disproportionate impacts of air toxics hazards across urban areas, such as those known as hot spots and minority and low-income communities. Air toxics pose special threats in urban areas because they are densely populated areas with a variety of toxic sources. These pollution sources combined could potentially pose significant health threats. Furthermore, minority and low income communities are often closely located to industrial and commercial urbanized areas indicating environmental injustice. ${ }^{46}$

Other programs under which VOCs are monitored are the Photochemical Assessment Monitoring Station Program, Chemical Speciation Network, and the Interagency Monitoring of Protected Visual Environments. The primary objective of the Photochemical Assessment Monitoring Station Program (PAMS) is to assess ozone control programs by identifying key constituents, tracking trends, characterizing transport, assisting in forecasting episodes, and assisting in improving emission inventories. The VOCs measured in the PAMS network include acetaldehyde, benzene, ethylbenzene, formaldehyde, styrene, toluene, and xylenes. State implementation plans are required to implement measures for monitoring ozone precursors. Title 40 of the Code of Regulations, Part 58 requires states to establish PAMS as part of their state implementation plan monitoring networks in ozone nonattainment areas classified as serious, severe, or extreme. ${ }^{47}$ The Chemical Speciation Network (CSN) supports the PM2.5 NAAQS. The purpose of the CSN is to provide nationally consistent speciated PM2.5 data for the assessment of trends. The CSN quantifies mass concentrations and PM2.5 constituents which include trace elements, elemental carbon, and organic carbon. ${ }^{43}$ The Interagency Monitoring of Protected Visual Environments (IMPROVE) program also provides PM2.5 speciation and mass measurements in national parks and wilderness areas. However speciated PM2.5 metals are the only toxics measured in this network. ${ }^{43}$ 
Finally, state and local air monitoring programs inform local air quality. The EPA began programs to fund local scale monitoring programs in the 2004 fiscal year. The goal of local air monitoring is to provide more flexibility to address middle and neighborhood scale issues that are not handled well by national networks. Specific objectives include identifying and profiling air toxics sources, developing and assessing emerging measurement methods, characterizing the degree and extent of local air toxics problems, and tracking progress of air toxics reduction activities. Projects are selected through an open competition process. Local scale monitoring is typically conducted for one and a half to three years. Expected outcomes of these projects are increased state, local, and tribal agency ability to 1) characterize the sources and local distribution of HAPS, and 2) assess human exposure and risk at a local scale. The program is referred to as the Community Scale Air Toxics Ambient Monitoring (CSATAM) projects. ${ }^{43,48}$

\section{Measurement of VOCs in Air}

Air toxics or HAPS are present in the atmosphere in gaseous, particulate, and semivolatile form and are therefore difficult to quantify with one single measurement method. Differences in chemical and physical properties of compounds further complicate quantification of HAPS. The choice of measurement technique depends on the objectives of data collection including chemical species of interest, funds available, and desired detection limit.

Measurement in air requires three main components: sample method, extraction method, and detection method. ${ }^{49}$ Sampling methods include active, passive, and grab sampling. Active sampling uses a pump to draw in a specified volume of air through an absorbent tube, at a constant, low flow rate. Active sampling is advantageous in long-term or regulatory settings due to the ability to constantly monitor. Passive sampling contains an absorbent material covered by cross-section tube and functions by diffusion. The distance between the cross-section and absorbent material determines the sampling rate. Grab sampling uses a stainless steel cannister with a flow restrictive inlet. Grab sampling is useful for short sampling times of 10-30 seconds in microenvironments.

Sample extraction is important to remove the sample for further analysis. Some common extraction methods are chemical and thermal desorption, solvent extraction, and solid phase 
microextraction. Chemical desorption uses organic solvent to desorb target VOCs from solid sorbent. Thermal desorption subjects the sorbent to high temperatures of $200^{\circ} \mathrm{C}$ to $380^{\circ} \mathrm{C}$ to remove the analyte. Passive sampler membrane extraction transfers analyte from a donor to an acceptor phase using a multi-membrane device.

Gas chromatography is the commonly employed method for VOC detection. Chromatography separates the analyte in a column containing liquid stationary phase. A carrier gas transports the analyte through the column to be eluted by polarity. The detector produces quantified measurements of peaks and retention times for speciated VOCs as they come off the column. Gas chromatography varies by detection method and includes mass spectrometer (MS), flame lonization (FID), thermal conductivity (TCD), electron-capture (ECD), atomic emission (AED), chemiluminescence (CS), and photoionization (PID). Depending on mixture sample, a specific detector may be more applicable. Mass spectrometers are the most beneficial due to their ability to identify unknown components and applicability to any sample. Flame ionization is commonly used for analysis of hydrocarbons of low molecular weight. Specifically, FID is unaffected by flow rate, noncombustible gases, and water, allowing high sensitivity and low noise. Electron capture detectors are highly selective and sensitive of electronegative groups. Photoionization detectors are selective of aromatic compounds, and inorganic and organic species and commonly used for air, water, and soil samples..$^{50}$

Measurement of VOCs in air is difficult due to the large variation in chemicals, multiple available techniques, and a lack of standardized and documented methods. The EPA has established a compendium of methods which can be found in Table 1-2. The methods are federally regulated monitoring methods and are considered a standard for measuring toxic organics in air. Monitoring occurs at multiple stations across the nation to assess trends, assess exposure, and to evaluate air quality models. The goal of air toxics monitoring is to support the reduction of exposure to HAPs. However, the data is not quite granular enough to estimate health effects as these ambient air concentrations are sparse, indirect measures of human exposure. 
Table 1-2. Toxic organic (TO) method for quantifying toxic organic compounds in air as defined by the Environmental Protection Agency.

\begin{tabular}{llll}
\hline Method No. & Compound & Sample Collection Device & Analytical Methodology \\
\hline TO-1 & VOCs & Tenax Solid Sorbent & GC/MS \\
TO-2 & VOCs & Molecular Sieve Sorbent & GC/MS \\
TO-3 & VOCs & Cryotrap & GC/FID \\
TO-4 & Pesticides/PCBs & Polyurethane Foam & GC/MD \\
TO-5 & Aldehydes/Ketones & Impinger & HPLC \\
TO-6 & Phosgene & Impinger & HPLC \\
TO-7 & Anilines & Adsorbent & GC/MS \\
TO-8 & Phenols & Impinger & HPLC \\
TO-9 & Dioxins & Polyurethane Foam & HRGC/HRMS \\
TO-10 & Pesticides /PCBs & Polyurethane Foam & GC/MD \\
TO-11 & Formaldehyde & Adsorbent & HPLC \\
TO-12 & NMOC & Canister or on-line & FID \\
TO-13 & PAHs & Polyurethane Foam & GC/MS \\
TO-14 & VOCs (nonpolar) & Specially-Treated Cannister & GC/MS and GC/MD \\
TO-15 & VOCs (polar/nonpolar) & Specially-Treated Cannister & GC/MS \\
TO-16 & VOCs & Open Path Monitoring & FTIR \\
TO-17 & VOCs & Single/Multi-Bed Adsorbent & GC/MS, FID, etc \\
\hline
\end{tabular}

Risk Assessment Framework

A major pillar of environmental health science is the risk assessment framework which is focused around the methods to evaluate exposure, predict health risks and outcomes, and inform decision making to control or otherwise respond to unacceptable exposures to environmental hazards. ${ }^{51}$ Human health risk assessment consists of four major parts: hazard identification, dose response assessment, exposure assessment, and risk characterization. Hazard identification 
determines whether the environmental chemical has the potential to cause harm to humans. Dose response assessment identifies the relationship between the dose of exposure and effect response. Exposure assessment investigates the source, frequency, and timing of exposure to the environmental chemical. Risk characterization quantifies and communicates the risk of the environmental chemical to human health. ${ }^{52}$

Exposure assessment aims to answer the questions of how much of the pollutant are people exposed to during a specific period and how many people are exposed. Exposure is the contact between a hazard in the environment and an individual, group, or population by inhalation, ingestion, dermal contact, or via placental transfer. ${ }^{53}$ The exposure assessment step identifies the affected population; calculates the amount, frequency, length of time, and route of exposure; identifies sources of containment; identifies exposure pathways and environmental fate; and finally, measures or estimates the dose or intake based on calculations. ${ }^{54}$ Exposure is the most important and least informed step in the hazard, exposure, and outcome pathway. Despite being the most informative pathway, exposure is often the weakest link with little information describing the hazard, exposure, or disease pathway. ${ }^{53}$

To measure exposure, researchers utilize direct or indirect measures of exposure. Direct measures of exposure are biological samples or personal monitoring and represent the gold standard of exposure assessment. Indirect measures of exposure are approximations of human exposure. Indirect measures of exposure include environmental samples and models. Direct and indirect methods of exposure assessment exist on a continuum of cost and precision (Figure 1-2). Indirect methods of exposure are often lower cost and approximate ambient exposure. Direct methods of exposure are more expensive and better approximate personal exposures. A comprehensive exposure assessment uses multiple methods to measure exposure, both direct and indirect.

As human beings, we interact with multiple microenvironments. We all interact with a global environment, and then we each interact with multiple microenvironments. Microenvironments are highly variable across individuals. Typical microenvironments are workplaces, school, indoor, and outdoor. Humans interact with each microenvironment at 
different rates and frequencies. The two major microenvironments are indoor and outdoor. While outdoor might be considered uniform, many pollutants vary spatially and temporally, as well as by nearby sources. Indoor environments are typically divided into work or school, or home environment. Thus, depending on the individual and their geographical region, an individual's exposure metric will be unique. Exposure assessment methods function to quantify the most appropriate exposure based on the primary microenvironment, or multiple microenvironments.

To assess the risk of a hazard to human health, it is important to assess exposure as accurately as possible using both direct and indirect monitoring, sampling of microenvironments, and consider tradeoffs. Exposure misclassification is a common source of bias in epidemiological studies. Additionally, exposure assessment in environmental epidemiology is a methodological problem due to long induction times between presumed causal action and incidence of disease..$^{55}$ Therefore, exposure assessment methods must function to reduce as much bias as possible.

Figure 1-3. Cost and exposure approximation tradeoffs in exposure assessment methods.

\begin{tabular}{|c|c|c|}
\hline & Personal & \\
\hline -Source Proximity & $\Lambda$ & -Biomonitoring \\
\hline -Questionnaires & & -Indoor \\
\hline & & Measurements \\
\hline No/Low & & Higher \\
\hline Added Cost $\leftarrow$ & & Additional \\
\hline -Existing Ambient Air & & -Air Quality Monitoring \\
\hline Monitoring & $\begin{array}{l}\Downarrow \\
\text { Ambient }\end{array}$ & -Land Use Regression \\
\hline & & -Community Monitoring \\
\hline
\end{tabular}




\section{Conceptual Framework}

Cardiovascular disease (CVD) is the leading cause of mortality worldwide, accounting for $31 \%$ of all deaths in $2015 .{ }^{56}$ In the United States, one in three adults have some type of CVD. By the year 2030 , approximately $44 \%$ of the US population is projected to have at least one form of CVD, of which the direct medical costs are projected to reach $\$ 818$ billion. ${ }^{57}$ Cardiovascular disease encompasses a group of diseases of the heart and blood vessels, which result from gene-environment interactions. ${ }^{58}$ Behavioral risk factors of CVD include unhealthy diet, physical inactivity, tobacco use, and excessive alcohol use. ${ }^{56}$ Although much effort has been invested in the prevention of CVD by reducing or ceasing behavioral risk factors, rates of heart disease continue to climb. Traditional risk factors neglect to account for $10-25 \%$ of CVD prevalence. ${ }^{59}$ Furthermore, risk factors and gene-environment interactions can act as modifiers which influence CVD penetrance. ${ }^{60}$ Cardiovascular disease is largely regarded as a behavioral disease dependent on lifestyle changes, yet recent evidence shows that environmental exposure plays a key role in disease incidence as well as clinical events. ${ }^{61}$ Thus, it is important to identify attributable risk factors, such as environmental chemicals, in CVD etiology; because genetic changes alone cannot account for the immense changes in disease incidence over short durations of time. ${ }^{62}$

In the 2010 update to the American Heart Association's scientific statement, authors gave evidence of a causal link between exposure to $\mathrm{PM}_{2.5}$ and CVD, establishing $\mathrm{PM}_{2.5}$ as a risk factor for the development of heart disease. ${ }^{6}$ In addition, the contributors to this statement released a call to action to better understand the role of other pollutants in the development of CVD, specifically of VOCs as constituents of particulate matter. The authors proposed three biological mechanisms by which exposure to environmental pollutants is linked to CVD. The three generalized intermediary pathways include 1) systemic oxidative stress and inflammation, 2) autonomic nervous system imbalance, and 3) transmission of PM or constituents into the blood. These generalized pathways were formulated on previous studies evidencing acute vascular dysfunction, chronic biological effects like atherosclerosis progression, and molecular level reactive oxygen species dependent pathways. 
This research aims to evaluate the relationship between exposure to pollutants like VOCs and CVD. We use the large review of PM and CVD and the proposed mechanisms as the basis for our research of pollutant exposure. Although there is a clear link between exposure to air pollution and cardiovascular disease, adverse effects of many gaseous pollutants such as VOCs, are less understood. Therefore, we hypothesize that VOC exposure occurs via similar biological pathways as PM. Already there is epidemiological research supporting a relationship between VOC exposure and CVD events such as heart failure, ${ }^{17}$ stroke, ${ }^{18}$ ischemic heart disease, ${ }^{18,} 19$ and cardiovascular mortality rates. ${ }^{20}$ Indoor exposure to VOCs has been associated with increased blood pressure and heart rate,,$^{21}$ and VOC metabolites measured in blood have been linked to an increased prevalence of doctor-diagnosed CVD. ${ }^{22}$ Similarly, HAPS released from industrial facilities are associated with cardiovascular mortality, ${ }^{19}$ and VOC-containing mixtures of pollutants such as automobile and diesel engine emissions, and tobacco smoke have been linked to the development of CVD. ${ }^{23-29}$

However, measuring exposure to VOCs is specifically challenging due to minimal routine air monitoring, ${ }^{11}$ spatial and temporal variability, ${ }^{14,20}$ and quick transformation to secondary pollutants in the atmosphere..$^{9,10}$ While exposure is the most important and least informed step in the hazard, exposure, and outcome pathway of the risk assessment process, exposure is often the weakest link with potential for bias in environmental epidemiological investigations. ${ }^{53}$ Therefore, our research utilizes multiple exposure assessment methods to assess the relationship between VOC exposure and CVD. To conduct impactful environmental epidemiological investigations of the relationship between exposure to VOCs and CVD, we will use source proximity, human biomonitoring, and mixture modelling. Given that exposure metrics vary in strength, new epidemiological studies must investigate relationships using multiple measures of exposure.

\section{Literature Review of Relationship between VOC Exposure and CVD}

Until now, we have identified 187 chemicals of concern classified as HAPs, of which several are VOCs. For the remainder of the work, we will focus on seventeen VOCs which are also classified as HAPs and are measured in the National Health and Nutrition Health Survey 
(Table 1-3). The seventeen included VOCs are acrolein, acrylamide, acrylonitrile, benzene, 1bromopropane, 1,3-butadiene, crotonaldehyde, N,N-dimethylformamide, ethylbenzene, ethylene oxide, propylene oxide, styrene, tetrachloroethylene, toluene, trichloroethylene, vinyl chloride, and xylene. Since CVD is our outcome of interest, we will focus on both direct, personal measures of exposure in observational and experimental human studies as well as indirect measures such as environmental monitoring or modeling and their link with CVD outcomes.

To review the literature, we entered the following search in NCBI PubMed database ((((volatile organic compounds) OR hazardous air pollutants) OR air toxics) OR industrial chemicals) AND cardiovascular disease. Eighty-three articles were chosen based on abstract and title content. These articles were then imported into the Health Assessment Workplace Collaborative platform (www.hawcproject.org). The HAWC project integrates and documents the overall workflow of a systematic literature review. ${ }^{63}$ The HAWC project has been adopted for use by the National Toxicology Program (NTP), the U.S. Environmental Protection Agency (US EPA), and the Texas Commission for Environmental Quality (TCEQ). From the 83 articles, 20 epidemiological studies were chosen after thorough review of abstracts. Articles were published between 1998 and 2019. Two were published in 1998 while the rest were published in the last ten years.

Volatile organic compounds are ubiquitous in the environment. Primary sources of anthropogenic VOCs include combustion, dry cleaners, hazardous waste sites, contaminated environmental media, and consumer goods. VOCs are classified by low molecular weight and high vapor pressure which subsequently allows quick vaporization into the air. Thus, the primary route of exposure to VOCs is inhalation. Due to spatial and temporal variation of VOCs, the literature includes several methods of approximating or measuring exposure in humans. Direct exposure measurement includes personal monitoring and biomonitoring. Indirect exposure measurement includes environmental or indoor monitoring, existing surveillance programs, occupational exposures, and proximity to sources. Of the 20 reviewed articles, 4 use direct exposure measurements and 16 use indirect exposure measurements. 
Table 1-3. Volatile organic compound parent names, urinary metabolite long names, short names, and estimated half-lives.

\begin{tabular}{|c|c|c|c|}
\hline Parent Compound & Metabolite & Short Name & Half-Life \\
\hline \multirow[t]{2}{*}{ Acrolein } & N-Acetyl-S- (2-carboxyethyl)-L-cysteine & CEMA & Unknown \\
\hline & N-Acetyl-S- (3-Hydroxypropyl)-L-cysteine & 3HPMA & $10 \mathrm{~h}$ \\
\hline \multirow[t]{2}{*}{ Acrylamide } & N-Acetyl-S-(2-carbamoylethyl)-L-cysteine & AAMA & $3.5 \mathrm{~h}$ \\
\hline & $\begin{array}{l}\text { N-Acetyl-S-(2-carbamoyl-2-hydroxyethyl)- } \\
\text { L-cysteine }\end{array}$ & GAMA & $3.5 \mathrm{~h}$ \\
\hline Acrylonitrile & N-Acetyl-S-(2-cyanoethyl)-L-cysteine & CYMA & $8 \mathrm{~h}$ \\
\hline $\begin{array}{l}\text { Acrylonitrile, vinyl } \\
\text { chloride, ethylene } \\
\text { oxide }\end{array}$ & N-Acetyl-S- (2-hydroxyethyl)-L-cysteine & HEMA & Unknown \\
\hline Benzene & trans, trans -Muconic acid & MU & $5 \mathrm{~h}$ \\
\hline 1-Bromopropane & N-Acetyl-S-(n-propyl)-L-cysteine & BPMA & Unknown \\
\hline \multirow[t]{3}{*}{ 1,3-Butadiene } & $\begin{array}{l}\text { N-Acetyl-S- (3,4-dihydroxybutyl)-L- } \\
\text { cysteine }\end{array}$ & DHBMA & Unknown \\
\hline & $\begin{array}{l}\text { N-Acetyl-S-(1-hydroxymethyl-2-propenyl)- } \\
\text { L-cysteine }\end{array}$ & MHBMA1 & $>9 h$ \\
\hline & $\begin{array}{l}\text { N-Acetyl-S-(4-hydroxy-2-buten-1-yl)-L- } \\
\text { cysteine }\end{array}$ & MHBMAЗ & $>9 h$ \\
\hline Crotonaldehyde & $\begin{array}{l}\text { N-Acetyl-S-(3-hydroxypropyl-1-methyl)-L- } \\
\text { cysteine }\end{array}$ & HPMMA & Unknown \\
\hline $\begin{array}{l}\mathrm{N}, \mathrm{N}- \\
\text { Dimethylformamide }\end{array}$ & $\begin{array}{l}\mathrm{N} \text {-Acetyl-S-(N-methylcarbamoyl)-L- } \\
\text { cysteine }\end{array}$ & AMCC & $23 \mathrm{~h}$ \\
\hline $\begin{array}{l}\text { Ethylbenzene, } \\
\text { styrene }\end{array}$ & Phenylglyoxylic acid & PGA & $6 \mathrm{~h}$ \\
\hline Propylene oxide & N-Acetyl-S-(2-hydroxypropyl)-L-cysteine & 2HPMA & Unknown \\
\hline \multirow[t]{2}{*}{ Styrene } & $\begin{array}{l}\text { N-Acetyl-S-(1-phenyl-2-hydroxyethyl-L- } \\
\text { cysteine + N-Acetyl-S-(2-phenyl-2- } \\
\text { hydroxyethyl)-L-cysteine }\end{array}$ & PHEMA & Unknown \\
\hline & Mandelic acid & MA & $2 \mathrm{~h}$ \\
\hline Tetrachloroethylene & N-Acetyl-S-(trichlorovinyl)-L-cysteine & TCVMA & $14 \mathrm{~h}$ \\
\hline Toluene & N-Acetyl-S-(benzyl)-L-cysteine & BMA & Unknown \\
\hline \multirow[t]{2}{*}{ Trichloroethylene } & N-Acetyl-S-(1,2-dichlorovinyl)-L-cysteine & 1,2DCVMA & $5.6 \mathrm{~h}$ \\
\hline & N-Acetyl-S-(2,2-dichlorovinyl)-L-cysteine & 2,2DCVMA & $5.6 \mathrm{~h}$ \\
\hline \multirow[t]{3}{*}{ Xylene } & $\begin{array}{l}\text { Urinary N-Acetyl-S-(dimethylphenyl)-L- } \\
\text { cysteine }\end{array}$ & DPMA & Unknown \\
\hline & 2-Methylhippuric acid & 2MHA & Unknown \\
\hline & $\begin{array}{l}\text { 3-Methylhippuric acid + 4-Methylhippuric } \\
\text { acid }\end{array}$ & $\begin{array}{l}\text { 3MHA \& } \\
\text { 4MHA }\end{array}$ & Unknown \\
\hline
\end{tabular}


Cardiovascular disease is a chronic condition encompassing a group of diseases of the heart and blood vessels, which result from gene-environment interactions. ${ }^{58}$ Modifiable and nonmodifiable risk factors contribute to the development of heart disease. Modifiable risk factors include physical activity, diet, obesity, high blood pressure, and blood lipids. Non-modifiable risk factors include age, sex, race or ethnicity, socioeconomic status, family history of CVD, diabetes, and environmental factors. Risk factors contribute to the development of hypertension, atherosclerosis, thrombosis, and ischemia resulting in cardiovascular events such as stroke, myocardial infarctions, heart failure, and eventually, mortality. Outcomes from the literature review included high blood pressure and heart rate, electrocardiograph changes, oxidative stress and inflammatory markers, measures of endothelial function, doctor diagnosed CVD, CVD events and hospital admissions, and mortality.

The major differences found in review were the methods of assessing relationships between exposure to VOCs and CVD outcomes. Classification of VOCs includes a wide range of chemicals difficult to assess individually or together. Many papers utilize dimension reduction techniques by further grouping VOCs into subclasses based on structural similarities, reactivity, benzene or substituted benzenes, BTEX compounds, or by using cluster or principal component analyses. Therefore, the exposure measurement varies widely throughout this review. The major themes of methods are 1) individual VOC 2) structural similarities 3) total VOCs or 4) principal component analysis. Principal component analysis is a technique often used to understand exposures to multiple components and the sources from which they are derived.

Six articles assessed the relationship between VOC exposure and blood pressure or hypertension. In a subset of the Detroit Exposure and Aerosol Research Study (DEARS), 63 participants wore personal exposure vests for six seasons to assess personal exposures to 12 VOCs. These exposures were further reduced to three principal components based on a primary petroleum source, a butadiene source indicating industrial exposure, and a freon source. Systolic blood pressure (SBP) was not significantly associated with any of the three principal components. Diastolic blood pressure (DBP) was consistently negatively associated with the butadiene principal component. Two large pregnancy cohorts, the Consortium on Safe Labor and the 
Consecutive Pregnancy Study, were used to assess the relationship between VOC exposure and gestational hypertension. Exposure to ambient VOCs was estimated with the Community Multiscale Air Quality Models (CMAQ). Nobles et al. found no significant associations with ambient VOCs and gestational hypertension, though there were increased risks for preeclampsia when estimating ambient VOCs. ${ }^{64}$ Contrary to Nobles, Zhu et al found significant associations for gestational hypertension and exposure to ambient ethylbenzene, toluene, and m- and o-xylene. ${ }^{65}$ Two studies in Taiwan measured indoor and personal exposure to total VOCs (TVOCs). In Taipei, 200 healthy homemakers were visited 12 times in two years. There were significant changes in blood pressure, a $4.32 \%(2.41,6.23)$ change in SBP per IQR $(0.65 \mathrm{ppm})$ of TVOCs and a $2.47 \%(1.13,3.81)$ change in DBP. ${ }^{66}$ From the cross-sectional study in Taiwan, TVOCs measured in the workplace were significantly associated with SBP, DBP and heart rate, independent of BMI. ${ }^{67}$ Finally, in a case-control occupational study, 345 workers were split among three exposure groups 1) benzene exposure, 2) xylene and benzene exposure, and 3) phenol exposure. Both SBP and DBP were significantly higher than controls. Arterial hypertension was prevalent in group $1,30.51 \%[\mathrm{OR}=2.44 ; 95 \% \mathrm{Cl} 1.24-4.85 ; \mathrm{P}=0.0054]$ and in group $2,27.92 \%$ $(\mathrm{OR}=2.00 ; 95 \% \mathrm{Cl} 1.11-3.61 ; \mathrm{P}=0.0136) .{ }^{68}$

Five papers assessed the relationship between VOC exposure and CVD biomarkers or ECG changes. Biomarkers of CVD are noninvasive measures of endothelial function, oxidative stress, clotting, or inflammation. These biomarkers can be useful sensitive, subclinical outcomes for CVD. In an occupational study, solvent and paint workers had significantly greater relative risks for cardiovascular effects of arrythmia, $\mathrm{P}$ wave changes, and QRS complex changes. ${ }^{69}$ Chuang et al found a $2.41 \%(1.63,3.19)$ change in inflammatory marker hs-CRP, and a $2.18 \%$ $(1.83,4.01)$ change in oxidative stress marker $8-O H d G$ per IQR $(0.665 \mathrm{ppm})$ of TVOCs. ${ }^{66}$ In the DEARS sub cohort, heart rate, flow mediated dilation (FMD) and basal arterial tone were positively associated with the petroleum source. Non endothelial mediated dysfunction was negatively associated with the petroleum source. ${ }^{70}$ Metabolites of BTEX compounds were assessed in an occupational cohort of Korean shipbuilding workers. Only toluene metabolites were significantly associated with the oxidative stress biomarker MDA. ${ }^{71}$ In a cross-sectional 
study of 300 healthy participants in Taiwan, indoor TVOCs were significantly associated with hsCRP $(19.7 \%$ 10.2,29.1), 8-OHdG $(3.8 \% 1.5,6.1)$, fibrinogen $(8.9 \% 4.2,13.6)$, the standard deviation of normal-to-normal intervals of heart rate variability $(-6.2 \%-9.1,-3.2)$, and the square root of the mean of the sum of the square of differences between adjacent normal-to-normal intervals of heart rate variability $(-2.6 \%-4.7,-0.5)$ in mixed linear models. ${ }^{72}$

In a subset of NHANES participants enrolled during 1999-2002, 2001-2002, and 20032004, $n=3,408$ participants were assessed for alkylbenzene metabolites in blood and their relationship to doctor diagnosed cardiovascular disease. Greater exposure to toluene was associated with greater prevalence of CVD (50-85th percentile OR 2.3, >85th percentile 3.49). Greater exposure to styrene was also associated with prevalence of CVD (50-85th percentile OR 2.03 , $>85$ th percentile 4.64). Additionally, greater exposure to Ethyl benzene (50-85th percentile OR .81, >85th percentile 3.1 ), M/p-xylene (50-85th percentile OR 1.12, >85th percentile 2.31 ), and O-xylene (50-85th percentile OR 1.49, >85th percentile 2.68) were associated with prevalence of CVD. ${ }^{22}$

Two papers assessed the relationship between VOC contaminated drinking water or domestic water and stroke. Benzene and trichloroethylene (TCE) contaminated drinking water occurred from contaminated hazardous waste sites on the Superfund National Priorities Lists. Participants were enrolled in the National Exposure Registry. On the basis of 60 cases of reported stroke, the prevalence odds ratios were 4.14 (1.54, 11.09; 2-12ppb TCE); 3.88 (1.4, 10.74; >12-60ppb TCE); and 3.2 (1.14, 8.99; >60-12,800ppb TCE). ${ }^{34}$ In Lybarger et al., the authors estimated the economic health burden associated with VOC contamination at Superfund sites. They found the excess prevalence rate for stroke was 5 per 1000 people and the excess prevalence rate for diabetes was 5 per $1000 .{ }^{33}$

Four papers assessed the relationship between ambient VOC exposures and cardiovascular events such as emergency department visits for CVD, emergency hospital admissions for heart failure, cardiovascular events at labor and delivery, and cardiovascular events at delivery admission. Two studies used the Consortium on Safe Labor to determine cardiovascular events at labor and delivery or at delivery admission. Both studies estimated 
ambient VOCs from the CMAQ. In Ha et al., there were increased odds of a cardiovascular event at labor and delivery for all VOCs at different lag times. However, models were not adjusted for other criteria pollutants such as PM. Specifically, 1,3-butadiene and cyclohexane had increased odds for the weekly average. ${ }^{73}$ Mannisto et al. found that recent exposure (5-day lag) to high ambient concentrations of some VOCs, specifically, exposure to ethylbenzene (OR $1.50,99 \% \mathrm{Cl}$ 1.08 to 2.09 ), m-xylene (OR $1.54,99 \% \mathrm{Cl} 1.11$ to 2.13 ), o-xylene (OR $1.51,99 \% \mathrm{Cl} 1.09$ to 2.09 ), p-xylene (OR 1.43, 99\% Cl 1.03 to 1.99) and toluene (OR 1.42, 99\% Cl 1.02 to 1.97), were associated with increased odds of cardiovascular events. ${ }^{74}$ In Hong Kong, exposure to benzene and alkynes was significantly associated with emergency hospital admissions for heart failure. ${ }^{75}$ Daily 24-hour average concentrations of VOCs were measured in the five county area of Atlanta, GA and split into groups of hydrocarbons (alkane, isoalkane, other alkane, cycloalkane, alkene, alkyne, aromatic) and oxygenates (aldehydes, acids, ketones). Almost all hydrocarbon groups were associated with cardiovascular ED visits. Oxygenates were not. ${ }^{76}$

Four studies assessed the relationship between ambient VOCs and cardiovascular mortality. Three studies estimated ambient VOC concentrations from fixed monitoring stations. The fourth study approximated exposure from industrial releases using the Toxic Release Inventory. In Hong Kong, benzene was significantly associated with a $4.1(1.0,7.4)$ and $5.8(1.0$, 10.8) percent excess risk in circulatory deaths per IQR of benzene for lag days 5-9 and 0-9. TEX compounds were also significantly associated with circulatory deaths $2.9(1.1,4.6)$ and $3.5(1.0$, 6.1) for lag days 5-9 and 0-9. ${ }^{77}$ In Taichung City, Taiwan, benzene, m,p-xylene, and o-xylene were all associated with all-cause mortality. Only benzene was significantly associated with cardiovascular mortality. ${ }^{78}$ In Ontario, Canada, adjusted relative risks showed weak positive associations for benzene, hexane, and total hydrocarbons with cardiovascular mortality. ${ }^{20}$ In the U.S., HAPS and CERCLA chemicals released from industry were significantly associated with cardiovascular mortality (1.87, p:0.0001 and 1.73 p:0.0001) and ischemic heart disease (1.57, $\mathrm{p}: 0.0001$ and 1.32, p:0.0001). ${ }^{19}$ 


\section{PROBLEM STATEMENT}

In our literature review, we found significant associations between several individual VOCs including acrolein, benzene, 1,3-butadiene, ethylbenzene, toluene, and xylene and cardiovascular outcomes like blood pressure, endothelial function, doctor-diagnosed CVD, and CVD mortality. Additionally, we found several significant associations between groups of chemicals like total VOCs, hydrocarbons, and component sources such as butadiene and petroleum, and cardiovascular outcomes. The reviewed works include both direct and indirect exposure assessment methods such as ambient air concentrations, indoor air concentrations, the $\mathrm{CMAQ}$, drinking water concentrations, source proximity, and metabolite concentrations from human biomonitoring. While there is some evidence of an association between VOC exposure and CVD, results are limited by cohort and exposure assessment method. Several studies assessed VOC exposure in exposed workers which may not represent real-world exposures outside the studied industry. ${ }^{79,80}$ The one study which examined direct measures of VOC exposure and doctor-diagnosed CVD in a nationally representative cohort used VOC metabolites in blood, those which are not as robust in capturing an individual's daily exposure. ${ }^{16,81,82}$ Studies using fixed monitoring stations and community modelling to assess VOCs in ambient air are limited by the sparsity of national coverage. ${ }^{83-85}$ Finally, several of these studies grouped VOCs by structure, source, or federal regulatory classification to improve interpretability. Given that there are more sophisticated and robust methods to assess exposure to individual pollutants and pollutant mixtures, we must employ more recently developed methods to better elucidate the role of environmental exposure in the development of CVD.

\section{PURPOSE OF THE STUDY}

The purpose of this study is to identify environmental stressors in the development of CVD using a multi-pronged approach to exposure assessment. Three methods of exposure assessment are used to evaluate the relationship between exposure to VOCs and CVD: 1) source proximity, 2) human biomonitoring, and 3) mixture modelling. Two populations are used in this study to evaluate VOC exposure. The first is a national population of the contiguous United States in which there is county-level data of industrial releases and age-adjusted, all cause and 
CVD mortality. The independent variable, industrial releases, and the dependent variable, mortality, are assessed longitudinally in an ecological study during the years 2002-2012. The second is a local population in Louisville, Kentucky in which there is individual-level urinary VOC metabolites and multiple CVD risk markers. The independent variables, the VOC metabolites, and the dependent variables, CVD risk markers, are assessed cross-sectionally. Finally, mixture modelling is used to assess multi-pollutant exposure in these two populations with two distinct methods: 1) the Bayesian Kernel Machine Regression, and 2) the variable selection procedure, elastic net.

The proposed study will contribute to the field by presenting more granular and mechanistic evidence of human exposure to environmental pollutants like VOCs and their role in the development of CVD. This study highlights the use of both aggregate-level and individuallevel human data to understand population and individual effects. The methods used to evaluate this relationship are more sophisticated than previous methods used, specifically the longitudinal evaluation and the newer mixture modelling methods. Finally, this study assesses multiple CVD outcomes that are mechanistic steps in the development of CVD as well as CVD endpoints like mortality. The study further illuminates the need for better environmental health and pollution regulations for improved public health.

\section{RESEARCH QUESTIONS AND HYPOTHESES}

For this dissertation, we hypothesize that exposure to environmental pollutants like VOCs contribute to the development of CVD. This study takes a three-pronged approach to exposure measurement to assess the link between VOC exposure and CVD. To measure and model exposure to VOCs, we use publicly available inventories of toxic chemicals collected and maintained by the federal government, human biomonitoring to quantify VOCs in urine, and machine learning and variable selection to model exposure to mixtures of VOCs. Our three specific aims include:

\section{Examine the relationship between county level toxic chemical releases and CVD} mortality. We will acquire publicly available data at the county level to create a dataset 
for an ecological analysis of the association between toxic releases and circulatory disease mortality. Variables will be obtained from CDC Wonder, EPA, IHEH, and the U.S. Census to accurately assess county level associations. Data will be acquired for consecutive years 2002-2012 in the contiguous United States. We will use mixed models to longitudinally assess fixed and random effects using the restricted maximum likelihood. We will create a county level risk score to account for chemical potency within the releases.

\section{Assess the relationship between individual level VOC metabolites and vascular} function. We will conduct a secondary data analysis of a cross-sectional cohort, the Louisville Healthy Heart Study. We will restrict the analysis to nonsmokers since tobacco smoke is a major source of VOCs. We will examine the associations between exposure to 17 individual VOCs and the classical cardiovascular risk factors. In addition, we will analyze the associations between exposure to individual VOC metabolites and biomarkers of vascular injury and dysfunction such as endothelial function and urinary metabolites of catecholamines, to assess the function and significance of these changes.

3. Apply multipollutant models to existing aims using new statistical methods. We will use statistical methods like the Bayesian Kernel Machine Regression (BKMR) to determine the effects of exposure to multiple VOCs on the development of CVD. BKMR flexibly models the exposure response function of multiple components on one health outcome, incorporating non-linearity and interaction among the mixture components. We will use the elastic net procedure to identify potential toxic chemicals associated with CVD mortality at the county level. Elastic net is a variable selection method which uses penalized regression to select more contributive variables.

\section{RATIONALE, RELEVANCE, AND SIGNIFICANCE OF THE STUDY}

An exposure assessment is the study of the distribution and determinants of substances and factors affecting human health. Exposure assessments address variable microenvironments 
using direct and indirect measures of exposure. Direct methods include biological monitoring and personal air monitoring. Indirect methods include exposure estimates and proxies such as environmental monitoring, modeling, and questionnaires. A combination of both direct and indirect methods provides a more complete view of exposure. Exposure assessments are a major factor of environmental epidemiology, the study of factors in the environment that are out of the individual's control, which often affect many people simultaneously. ${ }^{55}$

Exposure assessment in environmental epidemiology is a methodological problem due to long induction times between presumed causal action and incidence of disease. ${ }^{55}$ Exposure is the most important and least informed step in the hazard, exposure, and outcome pathway. The exposure metric is the estimate of exposure for each individual or group of the study quantified by using direct and indirect methods. Researchers must consider tradeoffs of cost and data availability in exposure quantification. To assess the risk of a hazard to human health, we assess exposure as accurately as possible with the data available and the most appropriate models. We use three methods to assess exposure: 1) source proximity, 2) human biomonitoring, and 3) mixture modelling.

In the first aim, we use source proximity to ecologically assess the relationship between industrial releases and CVD mortality in the contiguous United States. The Toxic Release Inventory (TRI) is a publicly available dataset which quantifies industrial releases by location and chemical each year, a beneficial proxy for exposure to VOCs. Due to the time series nature of the data, we were able to assess the relationship longitudinally in small area units known as counties across the United States. The spatial and temporal methodology improve the strength of the study. Data acquisition required no cost.

In the second aim, we use human biomonitoring to individually assess the relationship between urinary VOC metabolites and risk markers of CVD in a cross-section of time. Human biomonitoring of blood and urine plays a major role in assessing human health risk. Assessing exposures in biofluids offers a whole measurement of exposure, including absorption, adsorption, injection, ingestion, and inhalation. Urine analysis for VOC metabolites is advantageous due to longer half-lives of metabolites compared with parent compounds and the specificity of 
mercapturic acid metabolites. Urinary analysis is sensitive enough to detect background exposure from tobacco smoke or solvent exposure. ${ }^{86,87}$ Although exposures are acute, they offer a realtime perspective of prevalent chemicals in everyday environments.

In the third aim, we use statistical methods to model exposure to mixtures in our previous two aims. Although people are exposed to a myriad of environmental chemicals every day, epidemiological studies have historically assessed the relationship between one exposure and multiple adverse health effects. As a result, the impact of multiple exposures has not been well characterized. To combat this gap in the literature, we use two supervised methods to assess mixtures, a variable selection technique called elastic net, and a machine learning technique called the Bayesian Kernel Machine Regression in the previous two aims.

The proposed research uses a multi-pronged approach to assess exposure to VOCs and the relationship to CVD. There is a lack of critical information on the health effects of chemicals in our environment. Furthermore, new evidence suggests that there are no safe exposures and the effect of low level exposure on chronic diseases such as CVD. This project aims to gain information on cardiovascular effects of exposure to VOCs. Although much research has explored organ toxicity and carcinogenicity of VOCs, cardiovascular toxicity remains under researched. Additionally, there is deficient environmental monitoring of VOCs. Therefore, the project will assess how exposure to VOCs contributes to cardiovascular risk and disease rates using multiple exposure assessment methods.

The potential implications of results of this research are many. First, new methods of exposure assessment create new models of exposure to VOCs which better estimate the relationship to CVD. Traditional ordinary least squares regression is no longer the best estimate in assessing health effects. Here, we use mixed models for repeated measures, generalized linear models, penalized regression, and machine learning regressions, methods superior to traditional ordinary least squares estimation. Second, there is a deficiency in monitoring and quantification of VOCs in the environment. New information about environmental exposures to VOCs calls to action better surveillance and regulation of environmental pollutants. Third, we uncover more information about how the environment contributes to CVD, the leading cause of 
death in the world. These results implicate new CVD prevention methodology and require the need for more research.

\section{SUMMARY AND ORGANIZATION OF THE REMAINDER OF THE STUDY}

In summation, we know that CVD is the leading cause of death from environmental exposures. ${ }^{1}$ Environmental exposures are complex and infinite, targeting multiple routes of human exposure. Volatile organic compounds are pervasive environmental pollutants known to cause adverse health effects such as neurological effects, organ toxicity, and cancer. ${ }^{7,8}$ However, the role of VOC exposure in the development of CVD is less understood. The approximation of human exposure to VOCs is complicated by a lack of routine air monitoring of VOCs, ${ }^{11}$ large variations of VOCs in space and time,${ }^{13,14}$ and high chemical production each year. ${ }^{15}$ The goal of this study is to identify environmental stressors in the development of CVD using a multi-pronged approach to exposure assessment. Three methods of exposure assessment are used to evaluate the relationship between exposure to VOCs and CVD: 1) source proximity, 2) human biomonitoring, and 3) mixture modelling.

The following three chapters are individual studies using each exposure assessment method. Each of the three individual study chapters will commence with an introduction to the topic, the methods of the analysis, description of the results, and a discussion of the results. Chapter 2 describes the assessment of the longitudinal relationship between county-level toxic releases and all cause and CVD mortality. Chapter 3 describes the assessment of the crosssectional relationship between individual urinary VOC metabolites and CVD risk markers. Chapter 4 evaluates the role of mixtures of these exposures in the previous outcomes in Chapters 2 and 3, CVD mortality and blood pressure. Finally, Chapter 5 concludes the dissertation by summarizing results and generalizability of the three individual studies. 


\section{DEFINITION OF TERMS}

The following terms were used operationally in this study.

Cardiovascular Disease (CVD), the name for the group of disorders of heart and blood vessels, and include: hypertension (high blood pressure), coronary heart disease (heart attack), cerebrovascular disease (stroke), peripheral vascular disease, heart failure, rheumatic heart disease, congenital heart disease, and cardiomyopathies (World Health Organization 2021)

Air Pollution, a heterogeneous, complex mixture of gases, liquids, and particulate matter (Brook et al 2004)

Particulate Matter (PM), particulate matter ("thoracic particles" [PM10] $<10[\mathrm{mu}] \mathrm{m}$ in aerodynamic diameter, "fine particles" [PM2.5] <2.5 [mu]m, and "coarse particles" [PM10 to 2.5]) (Brook et al 2004)

Ozone $\left(\mathrm{O}_{3}\right)$, a highlight reactive, colorless-to-bluish gas with a characteristic odor associated with electrical discharges. $\mathrm{O}_{3}$ has been recognized since the 1950 s as the principal component of photochemical smog. In the troposphere, it is formed by the action of solar UV radiation on nitrogen oxides and reactive hydrocarbons, both of which are emitted by motor vehicles and many industrial sources. (Brook et al 2004)

Volatile Organic Compounds (VOCs), any compound of carbon, excluding carbon monoxide, carbon dioxide, carbonic acid, metallic carbides or carbonates, and ammonium carbonate, which participates in atmospheric photochemical reactions (40 CFR § 51.100)

Hazardous Air Pollutants (HAPs), Hazardous air pollutants, also known as toxic air pollutants or air toxics, are those pollutants that are known or suspected to cause cancer or other serious health effects, such as reproductive effects or birth defects, or adverse environmental effects (EPA 2017)

Exposure Assessment, a cornerstone of environmental epidemiology, the development of the exposure metric, the estimate of exposure for each individual of the study population (Weis et al 2005) 
Direct Methods, direct measurements obtained in external (to the individual's body) environmental media or through the determination of contaminants or their metabolites in biologic medium (Weaver et al 1998)

Indirect Methods, an indirect approach to exposure assessment which relies on validated models that evolve from well-characterized relationships between causative variables and exposure from studies using direct measurements (Weaver et al 1998)

Source Proximity, an indirect exposure assessment based on spatial analysis theory where location denotes the disposition of objects with respect to one another, in this case, distance. Distance is defined in terms of relationships and in qualitative terms. Proximity acts a proxy for frequency. Used for proximity to contaminant source. (Haining 2003)

Human Biomonitoring (HBM), a noninvasive process which provides an integrative perspective to one's whole, acute exposure, across all routes, and from all environmental media, and effective and useful method in quantifying risk (National Research Council 2006)

Mixture, three or more independent chemicals which cause multiple stressors. Stressors can include chemical and non-chemical stressors (NIEHS 2021)

Bayesian Kernel Machine Regression (BKMR), statistical approach that assumes linear and additive associations between each mixture component and health. The health outcome is modeled as a smooth function $(\mathrm{h})$, represented using a kernel function, of the exposure variables, adjusted for possible confounding factors. Variable selection is used to identify which of these components are responsible for the health effects of the mixture (Bobb et al 2015)

Elastic Net, penalized least squares regression method which shrinks coefficients toward zero but also has the ability of selecting grouped variables (Zou and Hastie 2005)

Cross-Sectional Study, observational study design which examines the relationship between a disease and an exposure among individuals in a defined population at a point in time (Aschengrau 2020)

Longitudinal Study, an observational or experimental study design which evaluates an association over time (Aschengrau 2020) 
Ecological Study, an observational study design which evaluates an association using the population rather than the individual as the unit of analysis (Aschengrau 2020)

Microenvironment, a component of the personal exposure assessment, the microenvironment is where a person's action takes place, at work, at home, commuting (Steinle et al 2013) 


\section{CHAPTER 2 COUNTY LEVEL TOXIC RELEASES ARE ASSOCIATED WITH}

CARDIOVASCULAR MORTALITY BETWEEN 2002 AND 2012

\section{INTRODUCTION}

Pollution is the largest environmental cause of disease and premature death in the world today. ${ }^{2}$ Air, soil, and water pollution and toxic chemical pollution are among the leading noncommunicable disease (NCD) risk factors globally; and are responsible for an estimated $71 \%$ of all NCD mortality. ${ }^{2}$ Cardiovascular diseases (CVDs) account for the majority of NCD deaths, ${ }^{88}$ as well as the majority of deaths from environmental exposures. ${ }^{1}$ In 2015 , pollution was responsible for $21 \%$ of deaths from all CVDs, $26 \%$ of deaths from ischemic heart disease, and $23 \%$ of deaths due to stroke..$^{89}$ Although there are several well quantified pollutants such as particulate matter, ozone, and lead, other known toxic chemical pollutants are poorly defined in their contribution to CVD mortality.

Toxic chemical pollution is a growing global problem. Between 1970 and 1995, production volume of synthetic organic chemicals tripled from 50 million tons to 150 million tons. ${ }^{90}$ Specifically, the United States Environmental Protection Agency (EPA) estimates 84,000 chemicals are in commerce from the Toxic Substances Control Act. ${ }^{91}$ Yet, thousands of these pollutants are not well monitored or quantified in the environment..$^{90}$ Furthermore, many of these chemicals have not been well-assessed for adverse health effects. The lack of surveillance presents a challenge to scientists in the assessment of adverse health effects from exposures to environmental chemicals.

The Toxic Release Inventory (TRI) may provide a remedy to understanding the effects of chemical pollution on mortality, although with limitations. The United States EPA launched the first pollutant release and transfer register known as the TRI under the Emergency Planning and Community Right to Know Act (EPCRA) of $1986 .{ }^{92}$ The TRI is a reporting system for facilities with 
a primary sector industry code included in the required reporting list, facilities employing ten or more people, or facilities manufacturing, processing or using EPCRA Section 313 chemicals. Qualifying facilities must report estimates of annual releases if they exceed thresholds of 25,000 pounds per year for manufacturing; 25,000 pounds per year for processing; or 10,000 pounds per year for otherwise use. There are currently 767 listed chemicals and 33 chemical categories covered under EPCRA Section 313. Chemicals reported in the TRI are classified as metals, Clean Air Act chemicals, or carcinogens and thus may have overlap in multiple categories. Although this list is not comprehensive, the TRI provides a starting point for assessing the burden of disease from toxic chemical pollution.

Previous studies have used the TRI to assess racial and sociodemographic disparities, ${ }^{93-}$ ${ }^{96}$ to describe toxic release trends and the need for risk communication, ${ }^{97-99}$ to assess cancer incidence, ${ }^{100-103}$ and to assess the association with mortality. ${ }^{19,} 104,105$ However, few studies have examined the role of toxic releases in cardiovascular disease, and none have examined the effect longitudinally. We hypothesize that changes in annual toxic releases are associated with changes in annual age-adjusted all cause and CVD mortality rates. Here, we conduct a longitudinal, ecological study by using the TRI to approximate county-level exposure and assess the relationship to all cause and CVD mortality in the contiguous United States from 2002 to 2012.

\section{METHODS}

\section{Study Design and Population}

Our overall objective was to assess the longitudinal relationship between county-level toxic releases from the TRI and age-adjusted, all cause, and circulatory disease (CVD) mortality in the contiguous United States. Our secondary aim was to create a risk score based on toxicity of the chemicals released and the relationship to mortality. This National County Level TRI Study (NCLTS) is a longitudinal ecological study measured at the small area or county level to assess the change in mortality over time in relation to hazardous chemicals. All data was publicly available at the county level and thus inexpensive and easily accessible. 
We initially intended to analyze the period between 2000 and 2018 for two reasons: First, the USA switched from the ninth revision to the tenth revision of the International Classification of Disease (ICD) system in 1999, so data from 2000 on would contain a consistent assignment of the medical cause of death. Second, data from this period would be the most recent and relevant information. However, due to the unavailability of other determinants of mortality, we trimmed the data to the period of 2002 to 2012.

\section{Data Sources}

We used data on deaths by underlying cause of death and county of residence from vital registration through the National Center for Health Statistics ${ }^{106}$ and on population from the U.S. Census Bureau ${ }^{107}$ for intercensal estimates of county-level percentages of age, sex, and race. From 2002 to 2012, there were a total of 31.8 million deaths in the contiguous U.S.; 11 million of these deaths were from circulatory disease (ICD-10 codes beginning with I) for which there is evidence of an association with toxic chemical pollution. ${ }^{2}$ Annual toxic chemical releases were acquired from the TRI database..$^{92}$ We downloaded national annual basic data files from the U.S. Environmental Protection Agency website for the consecutive years of 2002 to 2012 .

We also gathered publicly available data on other county descriptors that may affect mortality:

\section{$>$ Population and Housing Unit Estimates}

Population and housing unit estimates were downloaded from the United States Census Bureau $^{108,109}$ for the census years 2000 and 2010.

> Unemployment, Median Household Income, Education, and Poverty Estimates Unemployment, median household income, and education were published by the U.S. Department of Labor, Bureau of Labor Statistics, Local Area Unemployment Statistics (LAUS) and acquired from United States Department of Agriculture's Economic Research Service. Educational attainment for adults age 25 and older for the U.S. by state and county was published by the U.S. Census Bureau for the years 2000 and 2010. The data was acquired from the United States Department of Agriculture's Economic Research Service.

$>$ Alcohol and Smoking 
The Institute for Health Metrics and Evaluation (IHME) $)^{110}$ is an independent global health research center at the University of Washington. Results were published in 'Cigarette Smoking Prevalence in US Counties: 1996-2012' with prevalence estimates by county, year, and sex. ${ }^{111}$ Similarly, IHME published alcohol use prevalence estimates by county, year, and sex for 20022012. Estimates include any drinking, heavy drinking, and binge drinking. Estimates were produced by applying small area models to data from the Behavioral Risk Factor Surveillance System (BRFSS). Results were published in 'Drinking Patterns in US Counties from 2002 to 2012. ${ }^{112}$

$>$ Temperature and Heat Index

Temperature and heat index data were acquired from the CDC Wonder website. The North America Land Data Assimilation System (NLDAS) published daily air temperatures and heat index for the years 1979 to $2011 .{ }^{113}$ Data are available for the 48 contiguous United States plus the District of Columbia. We downloaded data from 2000 to 2011. The variables we include are the average daily maximum temperature in degrees Fahrenheit $\left({ }^{\circ} \mathrm{F}\right)$ and the average maximum daily heat index in ${ }^{\circ} \mathrm{F}$.

\section{$>\mathrm{PM}_{2.5}$ and Ozone}

Kim et al published Land Use Regression models of outdoor concentrations for PM2.5 and ozone $\left(\mathrm{O}_{3}\right)$ throughout the contiguous U.S for the years 1979-2015. ${ }^{114}$ Model estimates include annual average values for $\mathrm{PM}_{2.5}$ and the average during May through September of the daily maximum 8-hour moving averages for $\mathrm{O}_{3}$. The variables included were $\mathrm{PM}_{2.5}$ in micrograms per cubic meter $\left(\mu \mathrm{g} / \mathrm{m}^{3}\right)$ and $\mathrm{O}_{3}$ in parts per billion $(\mathrm{ppb})$. Data are available at national, state, county, and census tract. We acquired data from the Center for Air, Climate, and Energy Solutions (CACES) website.

\section{$>$ National Priority List Sites}

The U.S. Environmental Protection Agency published a list of all National Priority List sites as of February 2014. We acquired the data from Columbia University's Socioeconomic Data and Applications Center. ${ }^{115}$ Sites at the national level were accounted for. The frequency of sites per county ranged from zero to 23 . We created a categorical variable where a county with zero sites was classified as zero and a county with greater than zero sites was classified as one. 


\section{$>$ Rural Urban County Codes}

The NCHS developed a six-level urban rural classification scheme for U.S. counties and countyequivalent entities. ${ }^{116}$ The classifications are updated every ten years with the most recent in 2013. Counties are classified by four levels of metropolitan areas and two levels of nonmetropolitan areas. The classes of metropolitan areas include large central, large fringe, medium, and small metropolitan. The classes of nonmetropolitan areas include micropolitan and noncore.

\section{Units of Analysis}

There were 3,108 counties in the contiguous U.S. in 2010. A total of 2,603 counties had TRI chemical observations and recorded mortality rates between 2002-2012. The number of counties reporting each year ranged from 1,569 to 1,630 . There were $n=264$ unmatched observations due to no mortality counts. Therefore, we excluded the counties Broomfield CO, Campbell SD, Esmeralda NV, Eureka NV, Jackson SD, Oliver ND, and Sheridan MD.

We used the log-transformed, age-adjusted mortality rate for all cause and CVD as the outcome variables. We used two exposure variables to differentially assess toxic releases by volume and risk. The volume-based exposure variable was created by summing total facility releases by county and year. The risk-based exposure variable was created by summing the toxicity of the releases which was calculated by dividing the chemical volume by the reportable quantity $(R Q)$ or the toxicity equivalent score (TES) as reported by the Agency for Toxic Substances and Disease Registry (ATSDR). We refer to the volume-based predictor variable as the county sum $\left(\mathrm{C}_{\mathrm{ij}}\right)$ and the risk-based predictor variable as the risk sum $\left(\mathrm{R}_{\mathrm{ij}}\right)$. Both predictor variables were log transformed for analysis.

$$
C_{i j}=\sum T R_{i j}
$$

For each county (i), each year (j), we summed the total chemical releases in pounds (TR). 


$$
R_{i j}=\sum_{k}^{m} C R_{i j k} * \frac{1}{R Q_{k}}
$$

For each county (i), each year (j), we summed the product of each chemical release (CR) in pounds $(\mathrm{m})$ multiplied by the inverse of the RQ or the TES as determined by the ATSDR.

$k=1: m$ chemicals released $(C R)$, where $m$ is possible number of chemicals released $\mathrm{CR}=$ chemical released in pounds in each county each year $R Q=E P A$ reportable quantity or ATSDR toxicity environmental score

There are currently 767 individually listed chemicals and 33 chemical categories covered by the TRI program. Chemicals covered are those that cause cancer or chronic human health effects, significant adverse acute human health effects, and significant adverse environmental effects.

To account for the toxicity level of each chemical reported by amount, in each county, each year, we created a risk score.

The ATSDR creates a biannual point system ranking hazardous substances based on three criteria: the frequency of occurrence at a NPL site, of at least three or more sites, the toxicity as a reportable quantity (RQ) or toxicity equivalent score (TES), and the potential for human exposure based on the concentration of substances in environmental media at sites, and the exposure status of population at sites. We decided to use a regulatory agency developed value for toxicity to create our own risk score. Because TRI releases are not typically at Superfund or NPL sites, we could not use their definition of frequency or human exposure. Therefore, we used the toxicity equivalent to build our risk score. The toxicity component was developed by EPA to set RQs for hazardous substances as required by CERCLA Section 103(a). The EPA assigns CERCLA substances to one of five tiered RQ categories $(1,10,100,1000$, 5000) based on acute toxicity, chronic toxicity, carcinogenicity, aquatic toxicity, and ignitability 
and reactivity. For chemicals without $R Q s, A T S D R$ applied $R Q$ methodology to candidate substances to create a TES.

\section{Missing Data}

After merging of all variables and reduction to the years 2002 to 2012 , there were 17,700 observations from a total of 2,603 counties in the contiguous United States. There were no missing predictor variables for all observations. However, some counties report zero pounds or grams of releases. Reasons for zeroes include 1) facilities that report NA for a quantity on form R because the release or waste management quantity is not possible for that facility, 2) facilities do not respond to quantity questions and leave them blank which may have occurred more when paper reporting was still accepted, or 3) facilities may submit a form A certification statement which allows facilities to certify that they do not exceed annual releases of 500 pounds for a particular chemical.

A total of 1,015 counties (39\%), had at least one observation with a reported release of zero pounds. A total of 506 counties had zero values that occurred only once per FIPS code in 1,015 counties, approximately $50 \%$ of the counties. The second largest frequency was two zeroes per FIPS code; $27 \%$ of missing values. Because it is difficult to replicate analyses with $60 \%$ or more missing data, we excluded FIPS with seven or more missing observations for initial analyses. Therefore, we excluded four counties in total with seven or more missing observations. The final cohort includes 2,599 counties with repeated observations for the consecutive years 2002 to 2012 (Figure 2-1). 
Figure 2-1. Data acquisition flow chart for the National County Level TRI Study.

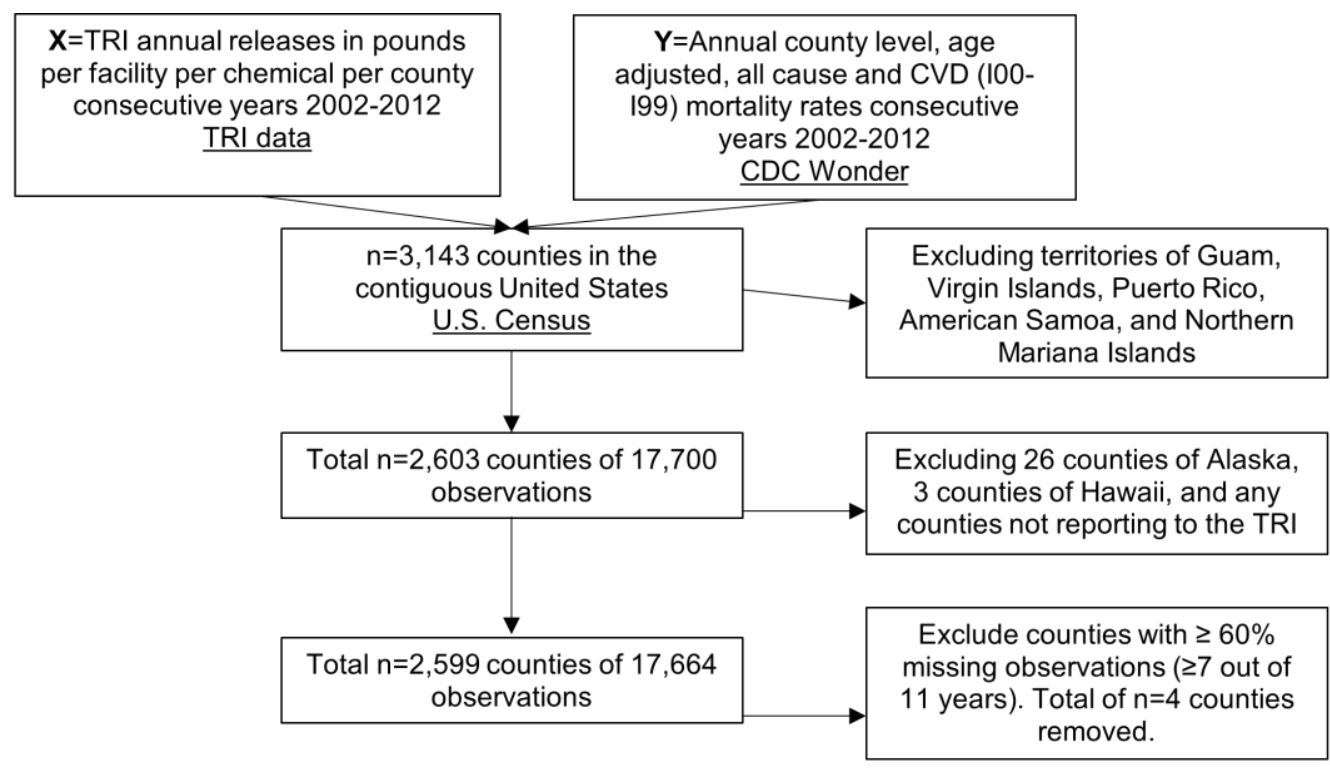

Primary Outcome: $\mathbf{Y}$; Primary Dependent Variable: $\mathbf{X}$

Covariates: Sex, Race, Median HH Income, Smoking, Alcohol, Education, Population/ Housing Density,

Temperature, Heat, NPL frequency, PM2.5

\section{Data Distribution}

County level toxic substance releases and calculated county level risk scores were gamma distributed and log transformed prior to analysis. Mortality rates were log normally distributed. County level demographic percentages were not normally distributed. We use the logit transformation of the demographic variables: percent male, percent White, percent Black, and percent Hispanic. Education of percent less than high school was normally distributed. Unemployment and poverty estimates were gamma distributed and log transformed. Median household income was gamma distributed and log transformed. County level alcohol use and tobacco use were normally distributed. Superfund NPL frequency was categorical and further dichotomized into counties with zero classified NPL sites or more than zero classified NPL sites. All environmental variables (PM2.5, ozone, maximum heat index, and temperature) were normally distributed. 


\section{Statistical Analyses}

This is a longitudinal ecological study. We use repeated measures mixed models where all variables are measured at the group level (the county-level), and the time in years. We use FIPS codes for each county as the subject identification number (ID). Observations are timeseries observations occurring for each consecutive year from the year 2002 to 2012 .

Mixed models examine and compare responses over time, estimating individual and population level regression parameters. Mixed models are preferred methodology over the traditional ordinary least squares method because they do not assume that observations are independent. Because time series data are measured on the same aggregate unit, the county, over a series of time points, observations are not likely independent. Mixed models account for this shortcoming by using maximum likelihood or restricted maximum likelihood (REML) to estimate effects. REML instead assumes that the subjects (the counties) are independent and that the observations within subjects (the annual measures) are correlated. REML reduces both type I and type II error rates. The model assumes that random effects and error terms are normally distributed and independent, and that the relationship between response variable and predictor variable is linear. ${ }^{117}$ Therefore, we used the general linear mixed model.

$$
y=X \beta+Z \gamma+\varepsilon
$$

$y$ is the vector of observed responses

$X$ is the design matrix of predictor variables

$\beta$ is the vector of regression parameters

$Z$ is the design matrix of random variables

$\gamma$ is the vector of random effect parameters

$\varepsilon$ is no longer required to be independent and homogenous

Within subject correlation is addressed by a series of covariance structures. Estimation in mixed models employs the use of the variance-covariance matrix. The covariance structure of the random effects is referred to as the $\mathrm{G}$ matrix. The covariance structure of the random errors is 
referred to as the $R$ matrix. The $G$ and $R$ matrices are estimated using the maximum likelihood method. Maximum likelihood estimation finds the parameter estimates that are most likely to occur given the data, ie, the joint probability of obtaining the data expressed as a function of parameter estimates.

Covariance structures model the variability in the data which cannot be explained by the fixed effects. They represent the background variability that the fixed effects are tested against. Covariance structures must be carefully selected to obtain valid references for the parameters of the fixed effects. To select the appropriate covariance structure, we must find a reasonable estimate for matrix $\mathrm{R}$, a block diagonal covariance structure where the block corresponds to the covariance structure for each subject. Observations outside the blocks are assumed to be independent. One must select the covariance structure that best fits the true covariance of the data. We want to know which component of variability is the dominant component. The components of variability include 1) random effects, 2) serial correlation, and 3) measurement error. Variograms can describe the association among repeated measures.

\section{Model Fitting}

To fit the model, we conducted an exploratory data analysis of the cross-sectional and longitudinal relationships in the data. We then fit a complex mean model by both backward elimination and forward addition of covariates. Once final covariates were decided, we output

OLS residuals to create a sample variogram to assess the appropriate covariance structure. ${ }^{118}$ The sample variogram had a process variance of 0.029 . The fitted line was horizontal with a slope close to zero indicating little serial correlation. Because the curve did not tend to zero as the time interval tended to zero, there seems to be some measurement error. Random effects were also observed in the sample variogram because the fitted line did not reach the process variance, indicating unexplained between subject variance. We chose the compound symmetry covariance structure because constant correlation is assumed regardless of the lag between pairs of repeated measurements. Compound symmetry is best for models with little to no serial correlation. We added the LOCAL statement to PROC MIXED to correct for measurement error and included a random intercept for between subject variability. Because the model would not 
converge, we removed the random intercept option from the model. Finally, we used the Akaike Information Criteria (AIC) to determine the best fit for each covariate addition or elimination after final selection of the covariate structure for each mortality outcome.

Data acquisition, merge, and data cleaning were conducted using tidyverse ${ }^{119}$ in $\mathrm{R}$ software (version 3.1.3), ${ }^{120}$ in addition to all figures and maps. Statistical analysis was performed using SAS version 9.4 (SAS Institute Inc., Cary, NC, USA).

Key assumptions and limitations of the methods

Our study is an ecological study and is thus prone to ecologic bias and nondifferential classification. Bias may be introduced by interaggregate variation in the disease rate among those not exposed to the risk factor, by groups acting as effect modifiers in the dose response relationship, or by confounding. Nondifferential misclassification may come from within group, ie within county, misclassification. Finally, the risk score that we created is only available for some, not all chemicals required to be reported under the TRI and therefore may reduce the number of observations.

\section{RESULTS}

The difference in population estimates between the baseline year 2002 and the final year 2012 are reported in Table 2-1. Mean values of county-level exposure variables, county sum, and outcome variables, all cause and CVD mortality rates, declined between 2002 and 2012, indicating a time-series association. However, the risk sum did not decline significantly. Population demographics changed significantly with a decline in percent White population and an increase in percent Black and Hispanic populations. Educational attainment, income, and poverty estimates were unchanged between 2002 and 2012, but unemployment levels increased. Mortality risk factors of smoking status and environmental risk factors of $\mathrm{PM}_{2.5}$ and $\mathrm{O}_{3}$ declined.

Hazardous waste sites and facilities releasing toxic chemicals prevail across the contiguous United States, especially in the eastern region and are concentrated in more populous areas (Figure 2-2). Between 2002 and 2012, there were 21,642 unique TRI facilities, with an average of 5,502 reporting each year. Additionally, there were 1,816 listed Superfund NPL sites. 
However, annual toxic releases declined by $34 \%$, from 70 million to 46 million pounds during this period (Mann Kendall trend p-value: 0.043). Similarly, age-adjusted, all-cause and CVD mortality rates decreased during this period, from 909.6 in 2002 to 807.7 in 2012 and from 344.7 in 2002 to 248.1 in 2012 (Mann Kendall trend p-value: 0.0003 and 2.6e-05), respectively (Figure 2-3).

Table 2-1. Descriptive statistics (number and median [minimum, maximum]) for counties in the contiguous U.S. for the baseline year, 2002 and the final year, 2012.

\begin{tabular}{|c|c|c|c|}
\hline Variable & 2002 & 2012 & $p$ \\
\hline $\mathrm{n}$ & 1616 & 1613 & \\
\hline County Sum (lbs) & 230665 (1872745) & $134585(567164)$ & 0.031 \\
\hline Risk Sum & $1434(32766)$ & $1418(27087)$ & 0.727 \\
\hline All Age Adjusted Mortality Rate & $909.58(127.68)$ & $807.69(133.41)$ & $<0.001$ \\
\hline CVD Age Adjusted Mortality Rate & $344.67(65.74)$ & 248.06 (56.99) & $<0.001$ \\
\hline$\%$ Male & $0.49(0.02)$ & $0.50(0.02)$ & $<0.001$ \\
\hline$\%$ White & $0.86(0.15)$ & $0.84(0.16)$ & 0.015 \\
\hline$\%$ Black & $0.11(0.15)$ & $0.11(0.15)$ & 0.406 \\
\hline$\%$ Hispanic & $0.06(0.10)$ & $0.09(0.12)$ & $<0.001$ \\
\hline Median Household Income ${ }^{c}$ & $38 k(9549)$ & $39 k(9640)$ & 0.998 \\
\hline \% Smoking & $27.07(3.58)$ & $23.27(3.95)$ & $<0.001$ \\
\hline$\%$ Alcohol & $49.03(12.67)$ & $51.10(10.95)$ & $<0.001$ \\
\hline \% Less Than High School & $21.61(7.80)$ & $21.20(7.71)$ & 0.902 \\
\hline \% High School Diploma & $34.41(6.83)$ & $34.21(6.90)$ & 0.996 \\
\hline$\%$ Some College or Associates & $26.27(5.12)$ & $26.59(5.08)$ & 0.891 \\
\hline$\%$ Bachelor or Higher & $17.72(8.07)$ & $18.00(8.23)$ & 0.999 \\
\hline \% Unemployment & $5.78(1.67)$ & $7.93(2.40)$ & $<0.001$ \\
\hline Population ${ }^{c}$ & $153(396)$ & $152(395)$ & 1 \\
\hline Average Daily Temperature $\left(\mathrm{F}^{\circ}\right)$ & $64.77(8.15)$ & $66.51(9.48)$ & $<0.001$ \\
\hline Average Heat Index $\left(\mathrm{F}^{\circ}\right)$ & $90.56(2.80)$ & $92.36(4.06)$ & $<0.001$ \\
\hline Ozone (ppb) & $52.79(6.08)$ & $48.97(5.57)$ & $<0.001$ \\
\hline PM2.5 $\left(\mu \mathrm{g} / \mathrm{m}^{3}\right)$ & $11.16(2.49)$ & $8.54(1.39)$ & $<0.001$ \\
\hline NPL Frequency ${ }^{d}$ & $0[0,19]$ & $0[0,19]$ & 0.997 \\
\hline
\end{tabular}

a Age-adjusted mortality rates are estimated at the county-level as per 100,000 people.

${ }^{\mathrm{b}}$ Age adjusted CVD mortality rate are circulatory disease mortality rates with ICD10 codes starting with I

${ }^{c}$ Measured in thousands $(\mathrm{k})$

${ }^{d}$ National Priority List hazardous waste sites are measured as median [minimum, maximum] 
Figure 2-2.Toxic Release Inventory (TRI) sites in dark blue and hazardous waste sites (NPL) in light blue in the contiguous United States.

TRI and NPL Sites in the Contiguous United States

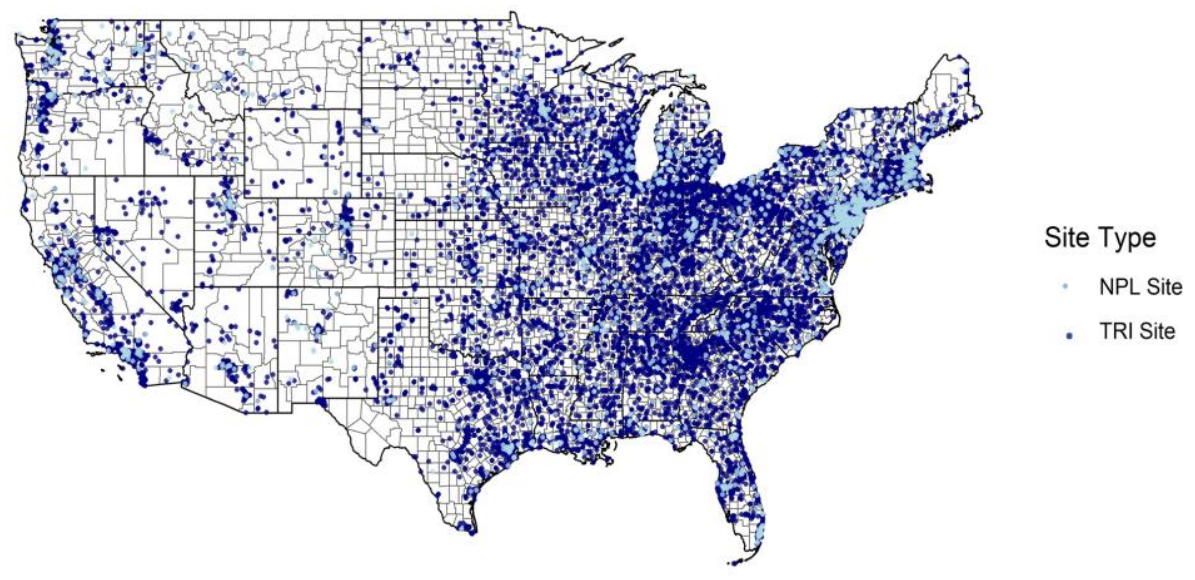

Figure 2-3. Secular trends in county-level TRI releases and All Cause and CVD Cause Mortality between 2002 and 2012.

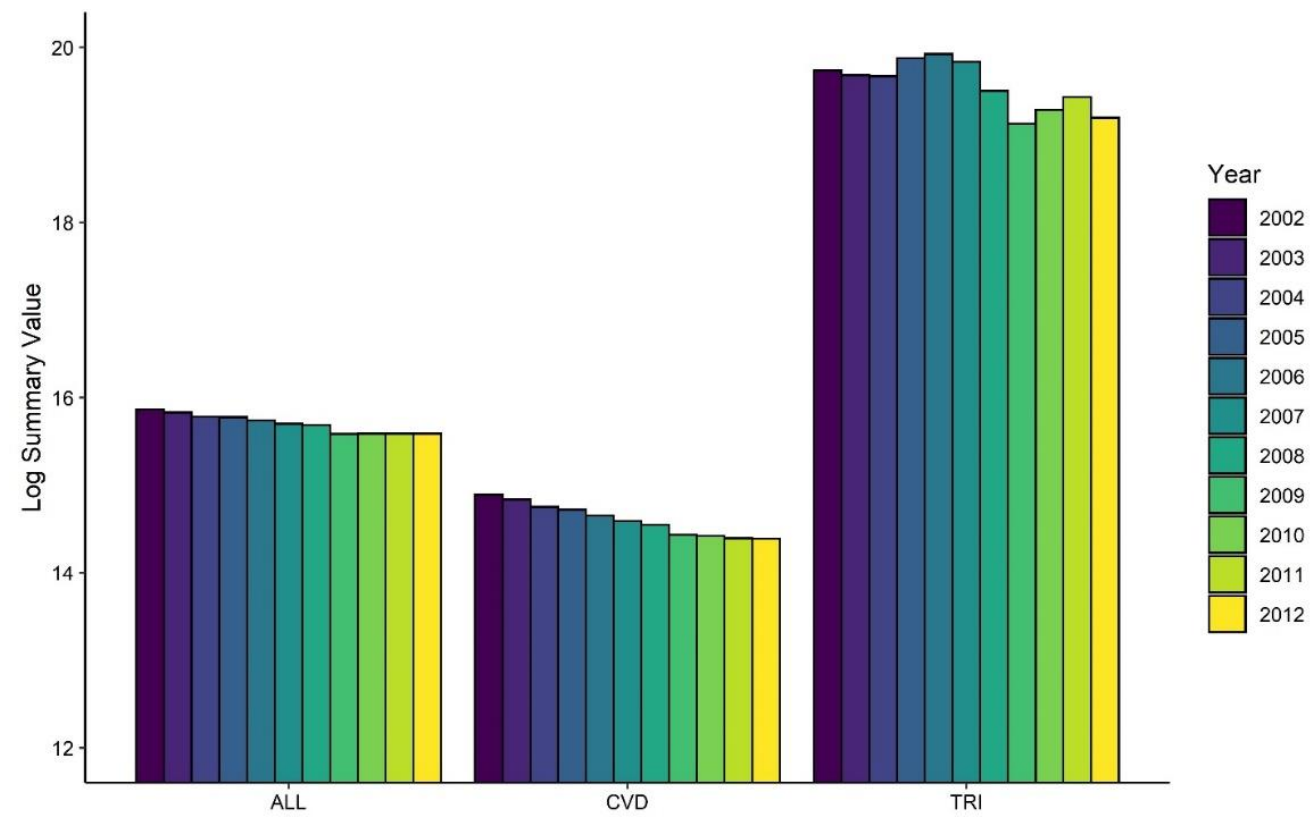


There are currently 767 listed chemicals and 33 chemical categories covered under EPCRA Section 313. The top chemicals released each year include metal compounds, acids, and solvents like ammonia, n-hexane, methanol, and toluene. Lead compounds were the most released compounds between 2002 and 2012 at a total of 762 million pounds and qualify as the most released Clean Air Act chemical, and metal (Figure 2-4). Styrene was the most released carcinogen.

Maps of median releases between 2002-2012 show variation is low between quantitybased releases and risk -based releases. Compared to the quantity-based map in panel A, the risk-based map shows few differences, specifically in the Gulf region in Louisiana and Texas, and in Utah and Nevada (Figure 2-5). Counties with high volume of toxic releases were not the same counties with high-risk scores. Calcasieu Parish, LA, Sumter County, AL, and Will County, IL released chemicals with the most risk in 2002, 2010, and 2011, respectively. Humboldt County, NV, Pinal County, AZ, and Salt Lake County, UT released the highest chemical volume in 2005, 2002, and 2006, respectively (Figure 2-6).

Both toxic release county sums and risk sums were significantly associated with ageadjusted all cause and CVD mortality rates between 2002 and 2012 (Figure 2-7). However, estimates from risk scores differed based on cause of mortality. For every $25 \%$ increase in county sum, we found a $1.7 \%(0.8,2.6 ; p$-value=0.0002) increase in all-cause mortality rate. For every $25 \%$ increase in risk sum, we found a $1.1 \%(0.2,2.0 ; p$-value $=0.0006)$ increase in all-cause mortality rate. For every $25 \%$ increase in toxic releases at the county level, we found a $2.8 \%(1.2$, 4.4; $p$-value $=0.0006$ ) increase in CVD mortality rate. Additionally, we found that for every $25 \%$ increase in county level risk score, there was a 3.0\% $(95 \% \mathrm{Cl} 1.3,4.6$; $\mathrm{p}$-value $=0.0132)$ increase in CVD mortality. Models were adjusted for intercensal demographic estimates of county percent male, percent White, percent Hispanic, and percent less than a high school diploma earned, unemployment, median household income, alcohol, smoking, frequency of National Priority List Superfund sites, and PM2.5. 
Figure 2-4. Top chemicals released at the county-level, annually, between 2002 and 2012 in the contiguous United States.

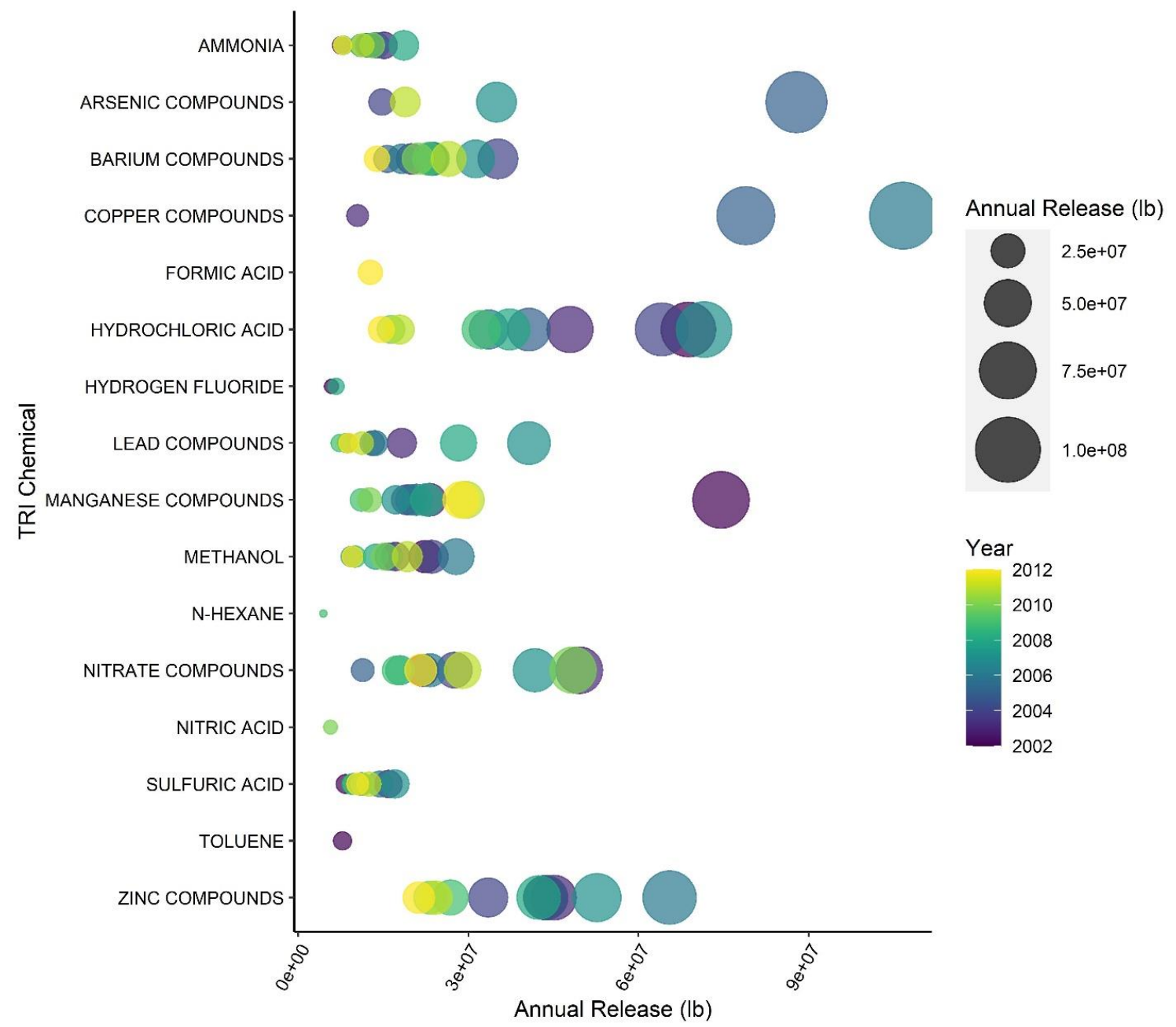


Figure 2-5. Comparison of county-level exposure indices, county sum and risk sum, in the contiguous United States.
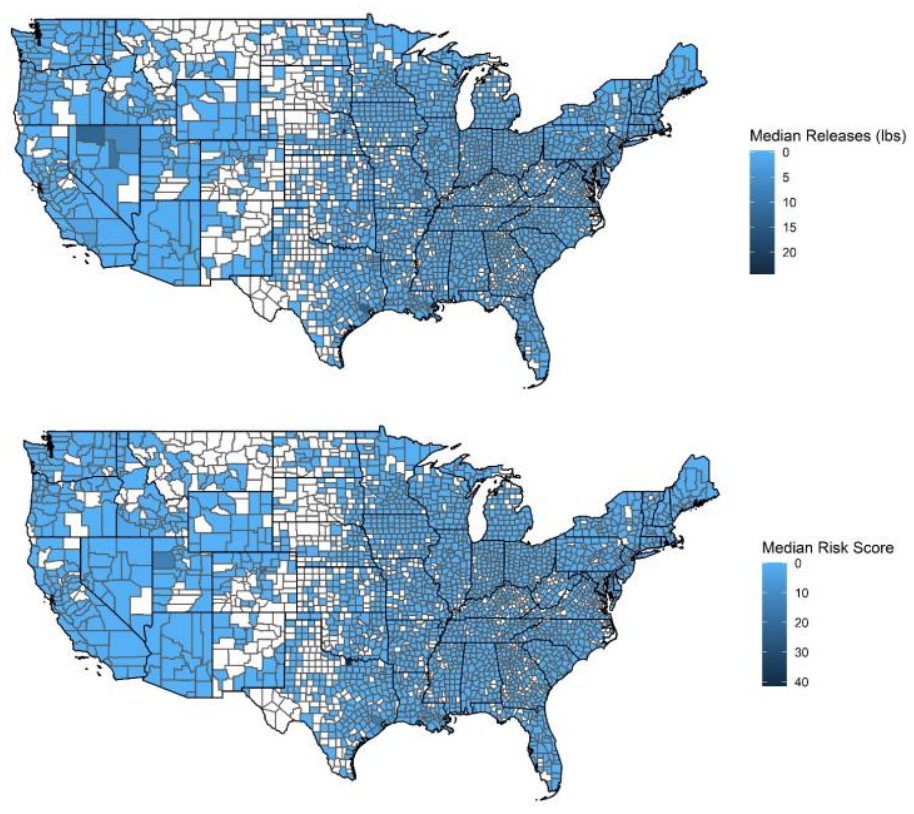

Figure 2-6. Comparison of counties with highest annual county sum and risk sum.

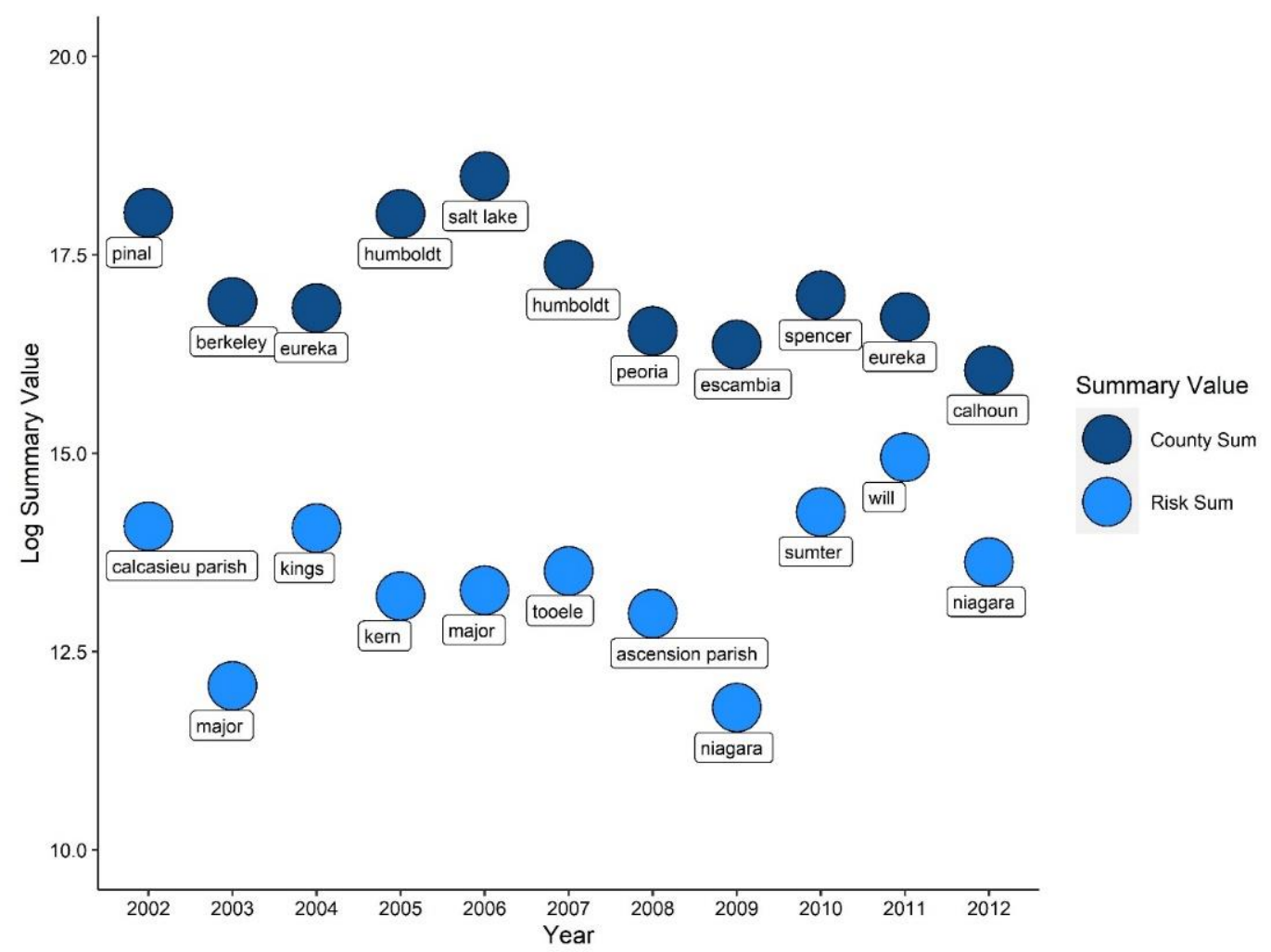


Figure 2-7. Percent increase in annual, county-level mortality rates (per $100 \mathrm{k}$ people) per $25 \%$ increase in county sum or risk sum.

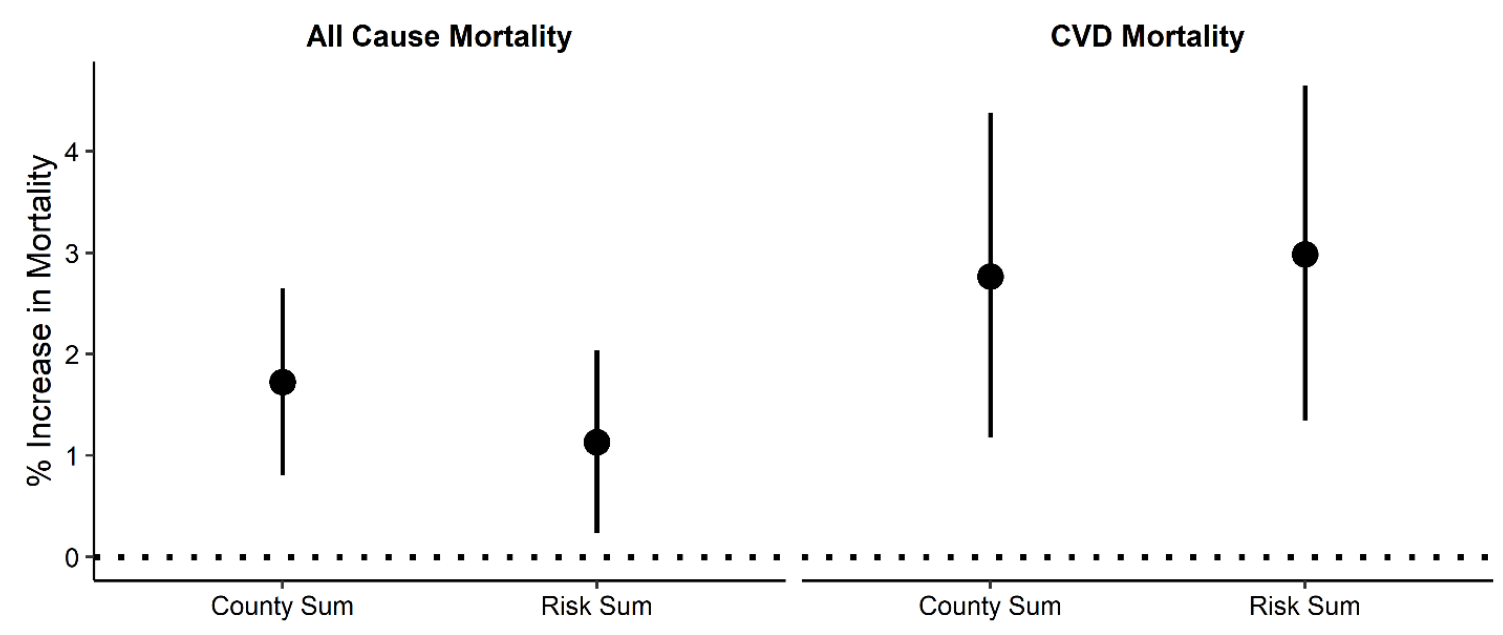

\section{DISCUSSION}

In our study, we described the top released chemicals between 2002 and 2012 as several acids, metals, and solvents, including lead, toluene, and styrene. We found significant associations between county-level toxic releases and both all cause and CVD cause mortality between 2002 and 2012 despite decreasing trends. County-level risk scores were also associated with both all cause and CVD cause mortality with a larger effect estimate in relation to CVD mortality. Counties releasing the highest risk chemicals were not the same as those releasing the highest volume of chemicals, indicating a clear need for better risk reporting to the TRI and more risk assessment for newer chemicals.

The TRI is a quasi-regulatory mechanism that provides publicly available data on toxic emissions and requires reporting to relevant environmental authorities. ${ }^{121}$ There are currently more than 700 chemicals and 33 chemical categories required to be reported under the TRI. Between 2002 and 2012, we found an overall trend decrease in TRI emissions. Consistent with these results, the TRI National Analysis reports overall declines except for years 2010 to $2012 .^{122}$ 
Similarly, the TRI National Analysis reported lead and lead compounds accounted for $98 \%$ of total releases in 2012 and substantially increased from 2009 to 2011 by $102 \% .{ }^{122}$ Overall, our descriptive analysis of the national TRI data between 2002 and 2012 followed the trends reported by the Environmental Protection Agency.

There is little reported evidence of the longitudinal association between county-level toxic releases and all-cause and circulatory disease mortality. To the best of our knowledge, Hendryx et al. was the only study to examine aggregate TRI releases across the U.S., and reported average annual, county-level TRI releases between 1990 and 1999 were significantly associated with higher all cause and cardiovascular mortality in 2006-2010. ${ }^{19}$ However, there are several reports of the association between CVD mortality and exposure to arsenic, ${ }^{123-125}$ cadmium, ${ }^{125,126}$ dioxins, ${ }^{127}$ lead, ${ }^{128}$ pesticides, ${ }^{129}$ and several other chemicals and metals which are covered under the TRI. Additionally, previous epidemiological studies have shown significant associations between exposure to volatile organic compounds and cardiovascular events ${ }^{18,20,75}$ and cardiovascular mortality rates. ${ }^{20}$ Thus, our study provides new models of national, ecological data to understand the longitudinal relationship between pollutants and population mortality rates.

In addition to our aggregate exposure measure, we created a risk score for each county to examine the effect of chemical toxicity on mortality rates. We found that our county-level risk scores were more strongly associated with CVD mortality rates than the toxicity independent exposure score. To create the score, we used a pre-existing toxicity component called the reportable quantity established by the ATSDR. Although there is an existing TRI risk score called the Risk Screening Environmental Indicators Model (RSEI), we created our own score for aggregation and to expand the toxicity included. Compared to the RSEI score, which is only based on chronic effects, the reportable quantities are based on acute toxicity, chronic toxicity, carcinogenicity, aquatic toxicity, and ignitability and reactivity. Furthermore, RSEI scores are only available for approximately 400 covered TRI chemicals. Previous studies have neglected to account for the TRI chemical risk level when modelling adverse health effects. ${ }^{97-99}$

Our ecological study has several limitations. First, although our ecological study is longitudinal, it is an observational study and cannot prove causation. Second, because the study 
uses an aggregate exposure and outcome variable, we cannot extrapolate the results to the individual level. Furthermore, the ecological design introduces ecological bias: bias due to interaggregate variation in the disease rate among those not exposed to the risk factor, bias due to groups acting as effect modifiers in the dose response relationship, and bias due to confounding variables. Finally, even though our study accounted for chemical toxicity, the values used were already established by the ATSDR. While advantageous to our development of a risk score, reportable quantities are often based on limited or dated toxicology studies and do not represent more sensitive cardiovascular endpoints.

Pollution is the largest environmental cause of disease and premature death in the world today. ${ }^{2}$ Yet the effects of pollution on non-communicable diseases like CVD have largely been underestimated. Particularly, the effects of chemical pollution on human health are poorly defined due to poor knowledge of many chemicals in common use. ${ }^{90,91,130-132}$ Pollution is a primary concern due to increases from globalization, technological industrialization, and urbanization. Furthermore, these increases greatly impact low- and middle-income countries, Black and Brown populations, and low-income communities in the U.S., and children.

In our study, we found that pollution defined by county-level toxic releases, in quantity and risk, are associated with county-level, age-adjusted all cause and CVD mortality rates in the contiguous United States between 2002 and 2012. The most released chemicals during this time were metal compounds, especially lead, solvents, and acids. Our study implicates the importance of pollution reduction to reduce deaths. Furthermore, it highlights the need to comprehensively understand pollution sources. Future studies must determine the most contributive chemicals both individually, and as mixtures. Even though our study used a composite value, there is value in parsing out contributions of individual chemicals. 


\section{CHAPTER 3 EXPOSURE TO VOLATILE ORGANIC COMPOUNDS IS ASSOCIATED WITH VASCULAR DYSFUNCTION}

\section{INTRODUCTION}

Cardiovascular disease (CVD) is the leading cause of death from environmental exposures, surpassing the number of attributable deaths to cancer mortality. ${ }^{1}$ The World Health Organization estimates that annually, exposure to air pollution is associated with 1.4 million deaths from stroke and 2.4 million deaths from heart disease, worldwide. ${ }^{3}$ To estimate the health effects of air pollution, most studies examine particulate matter of diameter $\leq 2.5 \mu \mathrm{m}\left(\mathrm{PM}_{2.5}\right)$ and tropospheric ozone $\left(\mathrm{O}_{3}\right) .{ }^{4}$ However, even though $\mathrm{PM}_{2.5}$ and $\mathrm{O}_{3}$ show strong associations between CVD progression and mortality, ${ }^{5-7}$ these pollutants represent only two components of complex real-world ambient air pollution, which in most locations around the world is a mixture of different pollutants, including oxides of nitrogen and volatile organic compounds (VOCs). Therefore, there is urgent need to assess the health impact of other commonly found pollutants, and to understand how they affect the risk and progression of cardiovascular and other diseases.

Volatile organic compounds (VOCs) are pervasive environmental pollutants and exposure to VOCs has been found to be associated with health effects such as CVD, neurological effects, organ toxicity, and cancer. ${ }^{16,36}$ Although these gaseous compounds are present across all environmental media, they are major components of indoor and outdoor air pollution due to high vapor pressure and low molecular weight. ${ }^{31,133,134}$ Primary sources of VOCs include tobacco smoke and vehicle exhaust, industrial releases, consumer goods production, products that contain organic solvents, and hazardous waste sites. ${ }^{8}$ VOCs also interact with other pollutants to create intermediates and secondary pollutants such as tropospheric $\mathrm{O}_{3}$ and secondary organic aerosols, a major component of fine particulate matter $\left(\mathrm{PM}_{2.5}\right)^{9}{ }^{9,}{ }^{10}$ However, compared with the wealth of epidemiological studies of $\mathrm{PM}_{2.5}$, the approximation of human 
exposure to VOCs is complicated by a lack of routine air monitoring of VOCs, ${ }^{11}$ large variations of VOCs in space and time, ${ }^{13,14}$ and high chemical production each year. ${ }^{15}$

The classification of multiple chemicals as VOCs further challenges the understanding of VOC exposure. Previous epidemiological studies have grouped ambient VOCs by alkenes, alkynes, and benzene, toluene, ethyl benzene and xylene (BTEX) compounds to show associations with cardiovascular events such as heart failure,${ }^{17}$ stroke,${ }^{18}$ ischemic heart disease,$^{18,19}$ and cardiovascular mortality. ${ }^{19,20}$ Total VOC exposure is often measured indoors and has been associated with increased blood pressure (BP) and heart rate,${ }^{21}$ heart rate variability, ${ }^{135}$ and autonomic nervous system changes. ${ }^{136}$ Individual VOC metabolites from human biomonitoring have been associated with doctor-diagnosed CVD ${ }^{22}$ and metabolic syndrome. ${ }^{137}$ Likewise, VOC-containing pollutants such as automobile and diesel emissions and tobacco smoke have been variably linked to the development of CVD and CVD risk. ${ }^{23,25,26,138}$ Nonetheless the role of exposure to VOCs in CVD pathophysiology in humans remain to be identified.

In addition to environmental monitoring, VOC exposures can also be monitored by measuring urinary metabolites of VOCs. The urinary metabolites of VOCs include specific, stable mercapturic acids with short physiological half-lives quantified by non-invasive sampling. ${ }^{86,139}$ Such measurements provide more proximal and individual-level assessments of VOCs. Therefore, in this study, we measured urinary VOC metabolites to assess the relationship between short-term exposure to individual VOCs and vascular outcomes. We assessed primary risk factors for CVD, BP and endothelial function, and sympathetic nervous system neurotransmitters/ catecholamines, which are reflective of stress leading to the development of CVD. ${ }^{6}$ Because personal monitoring and biomonitoring are considered the gold standard of exposure assessment, ${ }^{140}$ we measured urinary VOC metabolite levels to test the hypothesis that VOC exposure contributes to CVD risk in a nonsmoking, diverse, urban cohort. ${ }^{141}$

\section{METHODS}

Both exposure and outcome data were from a cross-sectional study designed to examine the relationship between exposure to environmental pollutants and CVD risk in an urban cohort. 
The Louisville Healthy Heart Study (LHHS) recruited 615 participants with moderate to high CVD risk (individuals undergoing primary or secondary prevention for CVD) at University of Louisville Hospital and associated clinics in Louisville, KY between October 2009 and April 2017. The primary prevention group refers to those who have known CVD risk factors (e.g. hypertension, hypercholesteremia, obesity, diabetes) that require management but who have no overt CVD. The secondary prevention group refers to those who have CVD risk factors that need to be treated, as well as overt CVD. Participants who met enrollment criteria gave informed consent and were administered a questionnaire to acquire baseline characteristics and demographic information. Inclusion criteria were: 1) age 18 years or older; and 2) treatment for CVD at the University of Louisville Hospital and associated clinics. Persons excluded were: 1) those unwilling to consent; 2) pregnant or lactating individuals; 3) incarcerated individuals; 4) persons with severe comorbidities (including lung, liver, kidney disease, cancer, and coagulopathies); 5) substance abuse; and 6) chronic cachexia. The Institutional Review Board at the University of Louisville approved the study.

\section{Measures of Vascular Function}

Blood pressure and endothelial function were measured at time of enrollment. Systolic and diastolic BP were measured after ten minutes of rest with an automated cuff and recorded as continuous variables. Three measurements were taken one minute apart with the last two measurements averaged. Peripheral endothelial function was measured in a subset of nonsmoking participants with and without diabetes $(n=70)$ to assess the role of endothelial function in the manifestation of diabetes. Participants with diabetes were classified by $\mathrm{HbA} 1 \mathrm{c}>$ $6.5 \%$, fasting plasma glucose $>126 \mathrm{mg} / \mathrm{dL}$, or random plasma glucose $>200 \mathrm{mg} / \mathrm{dL}$. Peripheral endothelial function was measured using fingertip peripheral arterial tonometry, i.e., EndoPAT, and calculated as a reactive hyperemia index $(\mathrm{RHI}) .{ }^{142}$

\section{Urinary Catecholamines and Metabolites}

Spot urine samples were collected at day of enrollment. Urinary catecholamines, monoamines, and their metabolites were quantified by ultra-performance liquid chromatography- 
tandem mass spectrometry (UPLC-MS/MS) as previously described. ${ }^{143}$ Briefly, frozen urine samples were thawed on ice, vortexed, and diluted (1:50) with $0.2 \%$ formic acid containing isotopically labeled internal standards. The method quantified epinephrine (EPI); norepinephrine (NE); dopamine (DA); serotonin (5-hydroxytryptamine, 5HT); metanephrine (MN); normetanephrine (NMN); 3-methoxytyramine (3MT); homovanillic acid (HVA); vanillylmandelic acid (VMA); and, 5-hydroxyindole-3-acetic acid (5HIAA) using a Waters Acquity Class-H UPLC coupled with Xevo TQ-S micro mass spectrometer. ${ }^{143}$ Analytes were normalized to urinary creatinine levels and reported as levels (nanograms of metabolite per milligram of creatinine, $\mathrm{ng} / \mathrm{mg})$.

Accuracy was measured by creating four levels of quality control (QC) samples of spiked, known amounts of analytes. Within and between run accuracy was measured in three independent runs with five replicates. Accuracy was calculated as the ratio of measured and expected concentration of analyte. The accuracy of within- and between runs of all analytes was $100 \pm 16 \%$. The within- and between run precision values, expressed as coefficient of variation (CV), were $<15 \%$ for all analytes, with the exception of 5-HT at LOQ level (17.3\%). Recovery was within the range of $92-113 \%$ at all spike levels (SD $\pm 16 \%$ ). Collectively, these results demonstrate excellent precision and accuracy for the determination of all the analytes in urine samples..$^{143}$

\section{Urinary VOC Metabolites}

Volatile organic compounds are ubiquitous environmental pollutants. Although there are several sources of VOCs, the major non-occupational source of exposure is tobacco smoke. However, VOCs can also arise from hazardous waste sites, vehicle exhaust, industrial releases, and household products. ${ }^{8}$ Urinary VOC metabolites are the preferred method to assess human exposure because of high spatial and temporal variability of VOCs in air, ${ }^{13,14}$ urine collection is non-invasive, and mercapturic acids are specific and stable with longer physiological half-lives than parent compounds. ${ }^{86}$ Given that half-lives of urinary VOC metabolites range from 2.1 to 34 hours, ${ }^{86}$ urinary VOC metabolites represent an individual's short-term or acute exposure. ${ }^{144} \mathrm{We}$ 
measured the most common urinary VOC metabolites, a well-developed panel of 22 metabolites of 17 parent VOCs ${ }^{86,139}$ in spot urine samples collected on the day of enrollment.

To assess VOC exposure, the levels of 22 urinary metabolites of 17 parent VOCs, (acrolein, acrylamide, acrylonitrile, benzene, 1-bromopropane, 1,3-butadiene, crotonaldehyde, $\mathrm{N}, \mathrm{N}$-dimethylformamide, ethylbenzene, ethylene oxide, propylene oxide, styrene, tetrachloroethylene, toluene, trichloroethylene, vinyl chloride, and xylene; were quantified using a modified version of the UPLC-MS method. ${ }^{86,145}$ In brief, urine was diluted with $15 \mathrm{mM}$ ammonium acetate and spiked with isotopically labeled internal standards. The analysis was performed on an Acquity UPLC core system coupled to a Quattro Premier XE triple quadrupole mass spectrometer with an electrospray source (Waters Inc, MA). ${ }^{145}$

The VOC metabolites were analyzed using a method adopted from CDC. ${ }^{86,139}$ The method was validated ${ }^{139}$ and the results showed that the sensitivity, precision, and accuracy of our method are comparable to the method reported. Specifically, the limit of detection (LOD) of each analyte is typically within 1 to $10 \mathrm{ng} / \mathrm{ml}$. Precision, assessed by the coefficient of variation of quality control samples, was within $17 \%$. Accuracy, assessed by analyzing spiked urine, was determined at three different levels of VOC metabolites, and was within 80 to $120 \%$. The method is highly reproducible, with relative standard deviations $<8 \%$. The sensitivity, accuracy, and precision were similar to those associated with the method developed by the CDC.

The primary exposure variables were 22 VOC urinary metabolites: N-Acetyl-S-(2carboxyethyl)-L-cysteine (CEMA), N-Acetyl-S-(3-hydroxypropyl)-L-cysteine (3HPMA), N-Acetyl-S(2-cyanoethyl)-L-cysteine (CYMA), N-Acetyl-S- (2-hydroxyethyl)-L-cysteine (HEMA), t,t-Muconic Acid (MU), N-Acetyl-S-(n-propyl)-L-cysteine (BPMA), N-Acetyl-S-(3,4-dihydroxybutyl)-L-cysteine (DHBMA), N-Acetyl-S-(4-hydroxy-2-buten-1-yl)-L-cysteine (MHBMA3), N-Acetyl-S-(3hydroxypropyl-1-methyl)-L-cysteine (HPMMA), N-Acetyl-S-(N-methylcarbamoyl)-L-cysteine (AMCC), Phenylglyoxylic acid (PGA), N-Acetyl-S-(2-hydroxypropyl)-L-cysteine (2HPMA), NAcetyl-S-(1-phenyl-2-hydroxyethyl)-L-cysteine + N-Acetyl-S-(2-phenyl-2-hydroxyethyl)-L-cysteine (PHEMA), Mandelic Acid (MA), N-Acetyl-S-(trichlorovinyl)-L-cysteine (TCVMA), N-Acetyl-S(benzyl)-L-cysteine (BMA), N-Acetyl-S-(1,2-dichlorovinyl)-L-cysteine (12DCVMA), N-Acetyl-S- 
(2,2-dichlorovinyl)-L-cysteine (22DCVMA), Urinary N-Acetyl-S-(dimethylphenyl)-L-cysteine (DPMA), 2-Methylhippuric acid (2MHA), and 3-Methylhippuric acid + 4-Methylhippuric acid (3,4MHA). Analytes were normalized to individual urinary creatinine levels to adjust for dilution and reported as levels (nanograms of metabolite per milligram of creatinine, $\mathrm{ng} / \mathrm{mg}$ ).

\section{Covariates}

Data on age, sex, race, body mass index, and self-reported tobacco exposure were collected at baseline using questionnaires. Information on medication use, cardiovascular history, and cardiovascular risk factors was obtained from medical records and questionnaires. We calculated the Framingham risk score (FRS), the risk for coronary heart disease, using the National Heart, Lung, and Blood Institute calculator. ${ }^{146}$ Because $\mathrm{PM}_{2.5}$ is an established CVD risk factor, ${ }^{5,6}$ we estimated $\mathrm{PM}_{2.5}$ exposure on the day of enrollment for adjustment in our models. We assessed the spearman correlation between $\mathrm{PM}_{2.5}$ and urinary VOC metabolites and found no significant correlations or correlations of $\rho>07$. Average daily $\mathrm{PM}_{2.5}$ values for all monitors in the Louisville Metropolitan Statistical Area were collected from the U.S. Environmental Protection Agency to estimate daily mean values of $\mathrm{PM}_{2.5}$.

\section{Statistical Analysis}

Smoking is a known risk factor for CVD, and a major source and possible confounder of VOC exposure. ${ }^{138,145,147}$ To assess environmental VOC exposure in individuals, we restricted the cohort to nonsmokers. We excluded individuals who were smokers, classified as individuals with urinary cotinine levels greater than $40 \mathrm{ng} / \mathrm{mg}$ of creatinine $(\mathrm{n}=222)$, despite self-reported positive smoking status. This range also excludes those exposed to secondhand smoke. ${ }^{148}$ Participants without measured VOC metabolite data $(n=46)$ and one participant with catecholamine outliers (six times the $99^{\text {th }}$ percentile of $21,633 \mathrm{ng} / \mathrm{mg}$ ) were removed from the analysis. The remaining cohort $(n=346)$ had $\leq 10 \%$ missing data for other covariates. Observations with unobserved outcomes were removed from models. Thus, the final cohort for each outcome was $n=308$ for BP, $\mathrm{n}=70$ for endothelial function, and $\mathrm{n}=323$ for catecholamines. To summarize these data, variables were stratified into low and high levels of total VOC metabolites. We created a 'total VOC score' 
by summing the Z-score of each of the log transformed and creatinine-corrected metabolites. Values were dichotomized into low and high groups, using the median value of summed Z-scores (-0.3143426). To determine significant differences between low and high total VOCs, we used Chi square tests for categorical variables and $t$-tests for continuous variables. Values of continuous variables are reported as mean \pm SD and categorical variables as $n(\%)$.

To examine the link between environmental VOC exposures and CVD risk, we constructed individual linear regression models by regressing normally distributed BP against each urinary VOC metabolite after adjusting for potential confounders. The levels of catecholamines and their metabolites, and $\mathrm{RHI}$ values were positive and right skewed; therefore, we used generalized linear models (GLMs) with a gamma distribution and log link function. Model adjustments were chosen a priori based on the literature for $\mathrm{BP}$ and $\mathrm{RH} \mathrm{I}^{6,56,62}$ and by creation of a directed acyclic graph. The relationships between levels of urinary VOC metabolites and BP were adjusted for age, sex, race, BMI, angiotensin converting enzyme (ACE) inhibitors, angiotensin II receptor blockers (ARBs), beta blockers, and $\mathrm{PM}_{2.5}$. Models including the covariates temperature, seasonal adjustment, median household income by census tract, diabetes, and cotinine did not change the effect estimate by more than $10 \%$ and were therefore not included in final models. The relationship between VOC exposures and RHI was adjusted for age, sex, race, BMI, diabetes status, and $\mathrm{PM}_{2.5}$. Additionally, we tested BP medications as moderators of the relationship between VOC exposures and BP, and diabetes and hypertension as moderators of the relationship between VOC exposures and $\mathrm{RHI}$. To simulate a doseresponse curve, we split the urinary VOC metabolite levels into equal tertiles and constructed adjusted generalized linear models of predicted means for each tertile as the new exposure variable to determine the shape of the exposure-response relationship. The lowest tertile concentration of each VOC metabolite was the reference group. To determine susceptible groups, we performed sub-analyses based on race and sex strata for blood pressure and catecholamine outcome variables. The data was not stratified by age due to high median age among the cohort. RHI was not included in sub-analyses due to small sample size. 
To examine the association between VOC exposures and catecholamines, monoamines, and their metabolites, we constructed generalized linear models by regressing each of the ten catecholamines against each urinary VOC metabolite. We built individual models for each VOC metabolite and adjusted for significant $(p<0.1)$ differences between low and high concentrations of each VOC metabolite. To determine susceptible groups, we performed sub-analyses based on race and sex strata.

To understand the relationship between exposure to VOCs and BP, we conducted a mediation analysis of VOCs and BP mediated by catecholamines. The mediation analysis was conducted by assessing the direct and indirect effects of each VOC metabolite ( $\mathrm{X}$ variable) on SBP and DBP ( $Y$ variables) with each urinary catecholamine $(\mathrm{M})$ as mediator. Analysis was conducted using the PROCESS macro in SAS. ${ }^{149}$ Additionally, we tested the exposure-mediator interaction in the relationship to BP.

Although there were multiple exposure variables, we did not correct for multiple testing to test the null hypothesis that all associations were null. ${ }^{150,151}$ Furthermore, this is an exploratory analysis where the VOC and CVD connection is not very strong. Thus, we wanted to minimize false negatives. We did not presume that all associations were due to random variation. Values are reported as $\mathrm{mmHg}$ or percent difference in outcome per interquartile range (IQR) of VOC metabolite concentration. Statistical analysis was performed using SAS version 9.4 (SAS Institute Inc., Cary, NC, USA) for individual VOC models of CEMA, 3HPMA, DHBMA, MHBMA3, and HPMMA, and R software (version 3.1.3) was used for graphical displays.

\section{Sensitivity Analysis}

We constructed models with and without normalization of VOC metabolites to creatinine. When the VOC metabolites were not normalized to creatinine, the creatinine variable was included as a covariate in the model. All models were used with the concentrations of VOC metabolites normalized to creatinine. Finally, 11 self-reported smokers were included despite cotinine values of $<40 \mathrm{ng} / \mathrm{mg}$. The maximum values of cotinine $(24.13 \mathrm{ng} / \mathrm{mg})$ for the self-reported smokers was much lower than the maximum values of self-reported nonsmokers $(38.10 \mathrm{ng} / \mathrm{mg})$. We conducted sensitivity analyses to determine changes in associations with and without self- 
reported smokers with no difference in result. Thus, we kept nonsmoker status using the above cotinine cut-off of $<40 \mathrm{ng} / \mathrm{mg}$.

\section{RESULTS}

In Table 3-1, we present descriptive statistics for the restricted non-smoking LHHS participants $(\mathrm{n}=346)$ after excluding smoking participants with urinary cotinine values $>40 \mathrm{ng} / \mathrm{mg}$ creatinine. Data for individual covariates were missing for $\leq 10 \%$ of participants. More than half the study population was White, about a third was Black, the mean age was 51.9 years, and the mean BMI was $32.9 \mathrm{~kg} / \mathrm{m}^{2}$. Participants with higher VOC metabolite concentrations were older, had higher Framingham risk scores, more comorbidities, and were prescribed more CVD medications.

At least $85 \%$ of all VOC metabolite observations in the nonsmoking population were above the LOD. All VOC metabolites were gamma distributed and highly variable. Boxplots of log transformed VOC metabolites normalized to creatinine are presented in Figure 3-1A. Women ( $n=198)$ had higher concentrations of DHBMA and HPMMA compared with men (Figure 3-1B); and White participants $(n=214)$ had higher concentrations of 3HPMA, DHBMA, and HPMMA compared with Black participants (Figure 3-1C). Parameter estimates were reported for IQR increase in each VOC metabolite: $88.3 \mathrm{ng} / \mathrm{mg}$ of creatinine for CEMA, $148.9 \mathrm{ng} / \mathrm{mg}$ for 3HPMA, 195.0ng/mg for DHBMA, 4.7ng/mg for MHBMA3, and 107.2ng/mg for HPMMA. Systolic and diastolic BPs and RHI had a log-normal distribution. The levels of catecholamines and metabolites were gamma distributed.

Figure 3-2A shows fully adjusted estimates for associations with systolic blood pressure (SBP), which were positive per IQR increase in 3HPMA $(0.98 \mathrm{mmHg}$ higher; $95 \% \mathrm{Cl}$ : 0.06, 1.91; $\mathrm{P}=0.038$ ). As shown in Figure 3-2B, 3HPMA was associated with SBP in an exposure dependent manner. Likewise, for each IQR of 3HPMA or DHBMA, there was a $-3.3 \%(95 \% \mathrm{CI}:-6.18,-0.37$; $\mathrm{P}=0.024)$ or a $-4.0 \%(95 \% \mathrm{Cl}:-7.72,-0.12 ; \mathrm{P}=0.012)$ difference in $\mathrm{RHI}$, respectively, indicating decreased endothelial function. Notably, $\mathrm{RHI}$ was lower in an exposure-dependent manner with DHBMA concentrations $(1.8 \% ; 95 \% \mathrm{Cl}: 1.65,2.06 ; \mathrm{P}=0.011)$. Although the reduction in absolute $\mathrm{RHI}$ index was similar between 3HPMA and DHBMA, it did not reach statistical significance for 
3HPMA. The other acrolein metabolite, CEMA, approached significance in the association with SBP (1.53mmHg; 95\% Cl: -0.27, 3.34; $\mathrm{P}=0.097)$ and $\mathrm{RHI}(4.3 \% ; 95 \% \mathrm{Cl}-10.0,0.9 ; \mathrm{P}=0.123)$. 
Table 3-1. Descriptive Statistics [number (\%) and mean ( \pm standard deviation (SD))] for nonsmoking participants in the Louisville Heathy Heart Study $(n=346)$, stratified by low and high VOC metabolite concentrations.

\begin{tabular}{|c|c|c|c|}
\hline Participant Characteristic & Low VOCa & High VOC & $p$-value ${ }^{b}$ \\
\hline Age & $49.3 \pm 11.9$ & $54.5 \pm 11.2$ & $<0.001$ \\
\hline Male & $96(56.1)$ & $102(59.3)$ & 0.63 \\
\hline Race & & & 0.8 \\
\hline Black (Black v Other) & $55(32.2)$ & $50(28.9)$ & 0.56 \\
\hline White (White v Other) & $104(60.5)$ & $110(64.0)$ & 0.24 \\
\hline Other (Other v All) & $13(7.6)$ & $12(6.9)$ & 0.84 \\
\hline BMI & $32.9 \pm 8.0$ & $32.8 \pm 8.1$ & 0.85 \\
\hline Daily Mean PM2.5 $\left(\mu \mathrm{g} / \mathrm{m}^{3}\right)$ & $12.9 \pm 5.0$ & $13.7 \pm 6.0$ & 0.26 \\
\hline Daily $\mathrm{Max}_{3}\left(\mu \mathrm{g} / \mathrm{m}^{3}\right)$ & $0.05 \pm 0.02$ & $0.05 \pm 0.02$ & 0.14 \\
\hline Temperature $\left(\mathrm{C}^{\circ}\right)$ & $12.4 \pm 13.2$ & $13.3 \pm 12.9$ & 0.55 \\
\hline Humidity (mg/L) & $22.3 \pm 10.7$ & $22.2 \pm 10.8$ & 0.91 \\
\hline Median Household Income ${ }^{d}$ & $49 \mathrm{~K} \pm 31 \mathrm{~K}$ & $45 \mathrm{~K} \pm 26 \mathrm{~K}$ & 0.22 \\
\hline Framingham Risk Score & $21.1 \pm 11.8$ & $25.3 \pm 9.1$ & 0.002 \\
\hline Hyperlipidemia & $77(45.6)$ & $102(59.6)$ & 0.013 \\
\hline Hypertension & $99(58.6)$ & $129(75.4)$ & 0.001 \\
\hline Diabetes & $44(26.0)$ & $62(36.0)$ & 0.06 \\
\hline Myocardial Infarction & $30(17.9)$ & $46(26.7)$ & 0.07 \\
\hline Stroke & $10(5.9)$ & $13(7.6)$ & 0.70 \\
\hline Heart Failure & $18(10.8)$ & $28(16.4)$ & 0.18 \\
\hline Beta Blockers & $60(35.9)$ & $88(52.4)$ & 0.003 \\
\hline Ace Inhibitors & $64(37.9)$ & $76(44.7)$ & 0.24 \\
\hline $\mathrm{ARBs}^{\mathrm{C}}$ & $11(6.6)$ & $20(11.9)$ & 0.14 \\
\hline Statins & $59(35.3)$ & $82(48.8)$ & 0.017 \\
\hline Aspirin & $60(35.9)$ & $83(48.8)$ & 0.022 \\
\hline Diuretics & $46(27.5)$ & $73(43.5)$ & 0.003 \\
\hline Vasodilators & $5(4.0)$ & $11(8.0)$ & 0.28 \\
\hline Calcium Channel Blockers & $34(20.4)$ & $40(23.8)$ & 0.53 \\
\hline
\end{tabular}

a Low and high VOC strata are dichotomized Z-score sums of the log transformed VOC metabolite concentrations normalized to creatinine $(\mathrm{ng} / \mathrm{mg})$.

${ }^{b}$ Continuous variables compared between strata by t-tests and categorical variables compared between strata by $X^{2}$ tests

${ }^{c}$ Angiotensin II Receptor Blockers

d Reported in thousands (K) 
Systolic and diastolic blood pressure in the entire cohort centered around 129.1 \pm 19.7 and 79.6 $\pm 11.1 \mathrm{mmHg}$, respectively. In comparison, Black participants had higher mean SBP $(135.6 \pm 23.0 \mathrm{mmHg})$ and DBP $(83.5 \pm 11.7 .0 \mathrm{mmHg})$ than White participants' mean SBP $(126.4 \pm 17.4 \mathrm{mmHg})$ and DBP $(77.9 \pm 10.4 \mathrm{mmHg})$. Compared with the relationship between 3HPMA and SBP found in the total cohort, Black participants showed a larger association with SBP (5.1 mmHg higher; $95 \% \mathrm{Cl}: 1.5,8.8 ; \mathrm{P}=0.006)$, despite lower levels of urinary 3HPMA in this population (Figures 3-1C and 3-2C). Additionally, women had a larger association with SBP per IQR of 3HPMA (1.2 mmHg higher; 95\% Cl: 0.08, 2.4; $\mathrm{P}=0.038)$. In White participants, estimates were higher for SBP $(0.8 \mathrm{mmHg}$ higher; $95 \% \mathrm{Cl}: 0.05,1.5 ; \mathrm{P}=0.037)$ and diastolic blood pressure (DBP) (0.6 mmHg higher; 95\% Cl: 0.2, 1.0; $\mathrm{P}=0.007)$ per IQR increase in MHBMA3 (Figure 32C).

Several catecholamines were significantly associated with VOC metabolites. Urinary EPI levels were positively associated with IQR increases in MHBMA3 (3.1\% higher; $95 \% \mathrm{CI}: 0.5$, 5.7\%; $\mathrm{P}=0.018$ ) and HPMMA (0.04\% higher; 95\% Cl: 0.0, 6.6\%; $\mathrm{P}=0.036)$ (Figure 3-3). Urinary DA levels were positively associated with IQR increases in HPMMA (3.3\% higher; $95 \% \mathrm{Cl}: 1.1$, 4.4\%; $P=0.001$ ). In Black participants, urinary NE and NMN levels were positively associated with IQR increases in DHBMA (15.7\% higher; 95\% Cl: 5.6, 27.0\%; $\mathrm{P}=0.001$; and 11.6\% higher; 95\% Cl: 3.7, 20.0\%; P=0.002) compared with the entire cohort. DA and serotonin end products, HVA and 5HIAA, were positively associated with 3HPMA, DHBMA, MHBMA3, and HPMMA.

To understand whether catecholamines modify or mediate relationships between VOC metabolites and markers of vascular dysfunction, we tested for both interaction and mediation. Mediation analyses showed null results. Although we observed significant direct effects between the acrolein metabolites and catecholamines, there were no significant indirect effects of catecholamines mediating the effects of VOC exposure on blood pressure. However, EPI levels were shown to modify the associations of MHBMA3 with DBP. Similarly, DA levels modified the association between HPMMA and SBP (data not shown). There was a positive association between MHBMA3 and DBP (0.5\% higher; $95 \% \mathrm{Cl}: 0.0,1.0 \%)$ in participants with higher urinary 
Figure 3-1. Exposure distributions of each VOC metabolite, CEMA, 3HPMA, DHBMA, MHBMA3, and HPMMA, reported as totals $(A)$, and stratified by sex $(B)$ and race $(C)$.
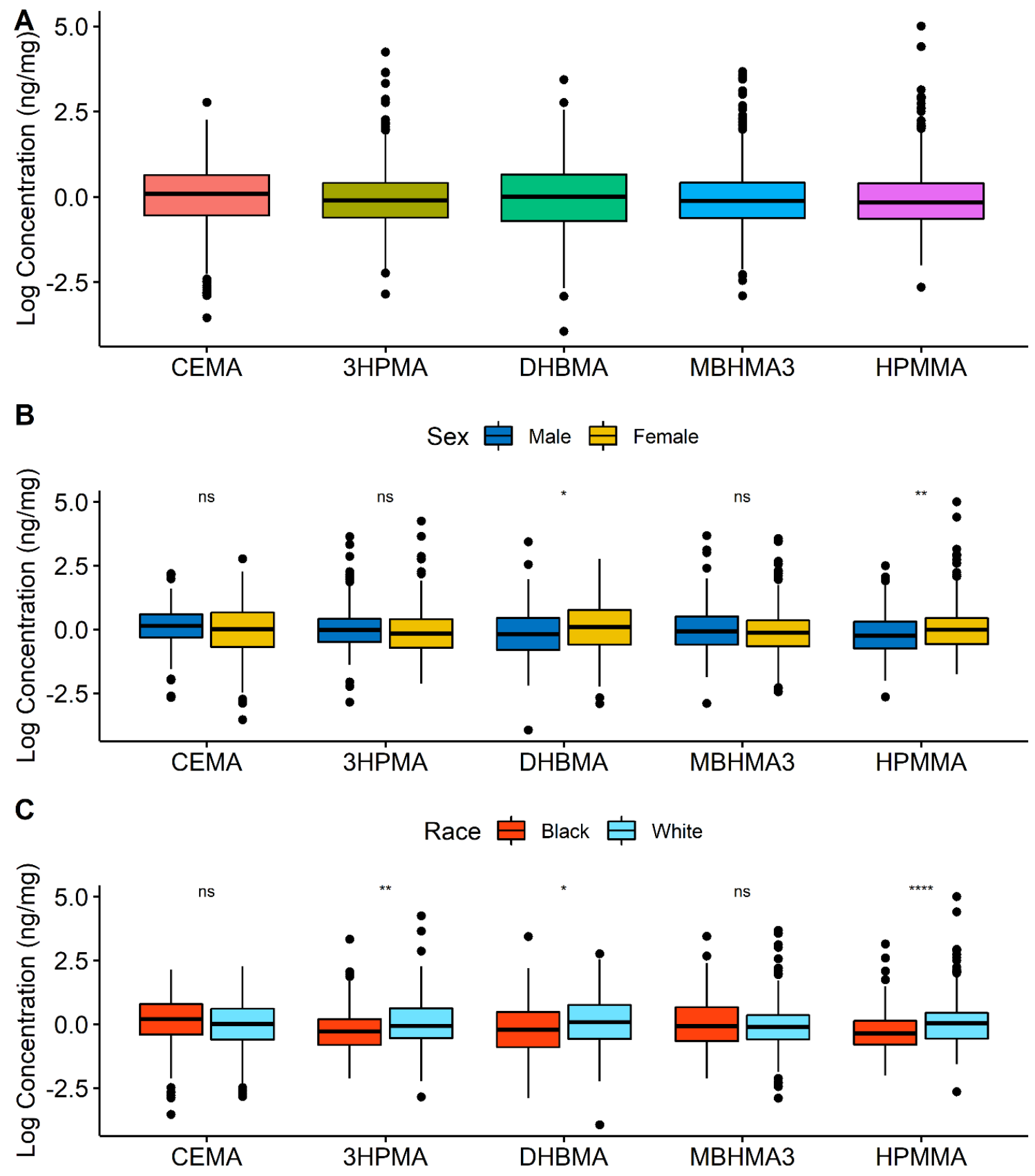

Values are reported as the log of the concentration of creatinine corrected urinary VOC metabolites (ng/mg creatinine). Wilcoxon unpaired two sample tests used to compare means. Single asterisk indicates $p$-values $<0.05,3$ asterisks indicate $p$-values $<0.001$, and $n s$ indicates not significant, $p$-values $>0.05$. 
Figure 3-2. Differences $(\mathrm{mmHg})$ and percent differences, and 95\% confidence intervals in blood pressure and RHI per IQR of VOC metabolite (A). Predicted means and 95\% confidence intervals $(\mathrm{mmHg})$ in blood pressure and \% difference in $\mathrm{RHI}$ with increasing tertile of 3HPMA and DHBMA (B). Differences and 95\% confidence intervals $(\mathrm{mmHg})$ in blood pressure, stratified by sex and race $(\mathrm{C})$.

A

Systolic

Diastolic

$\mathrm{RHI}$

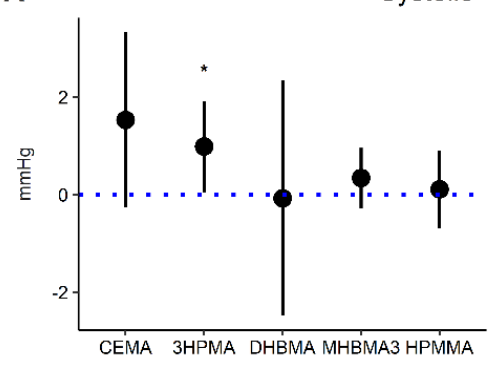

B
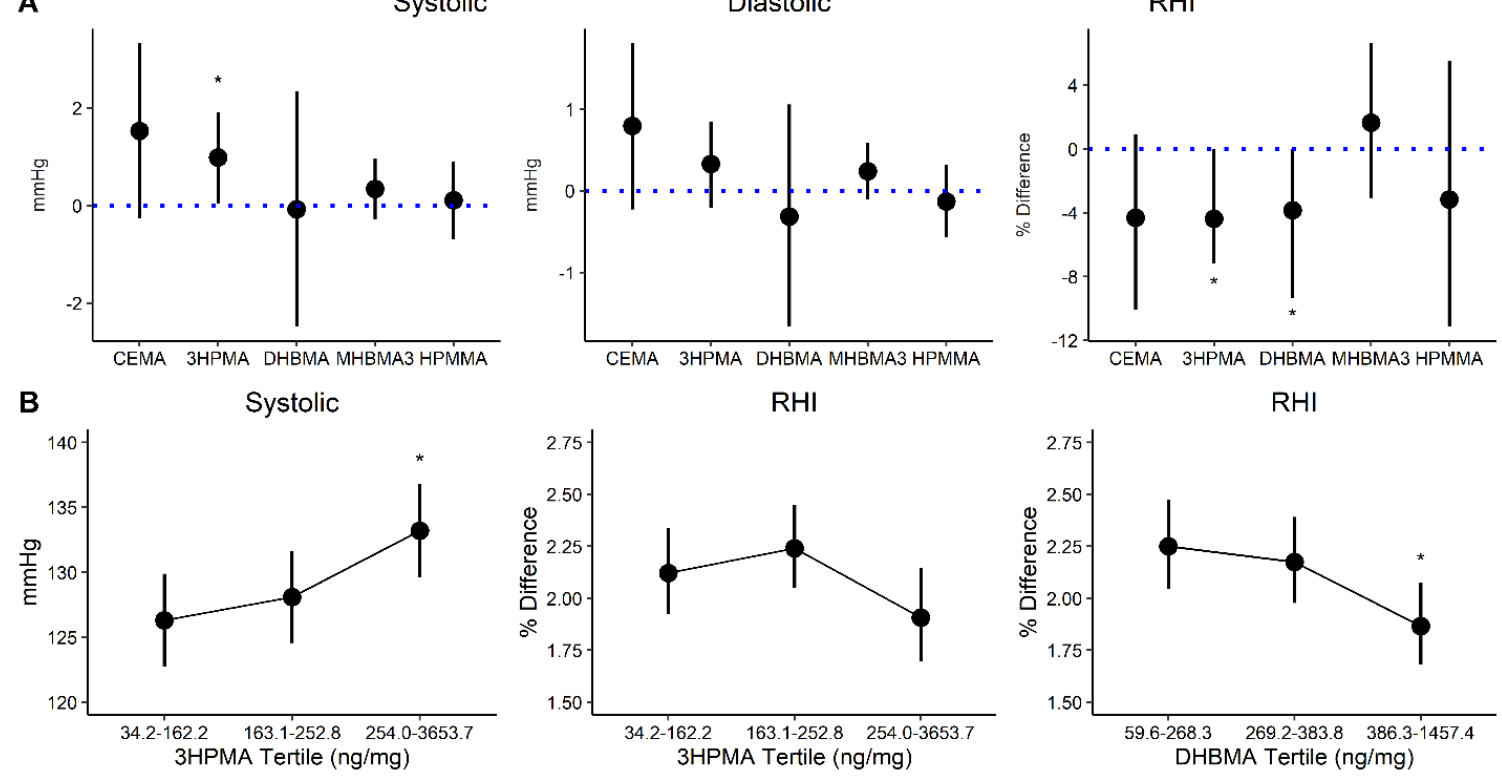

C

Systolic

Diastolic
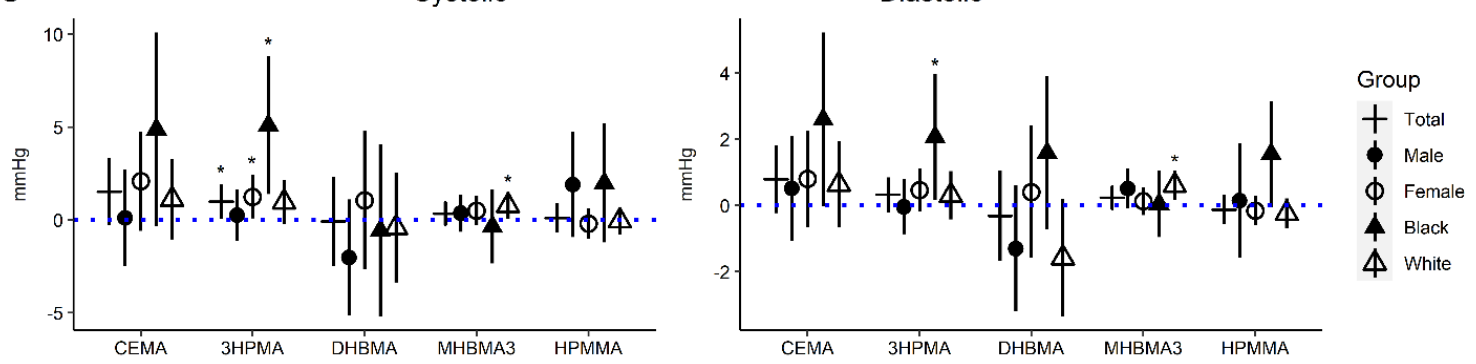

Model adjustments for blood pressure $(\mathrm{n}=308)$ include age, sex, race, BMI, daily $\mathrm{PM}_{2.5}$, ace inhibitors, ARBs, and beta blockers. Model adjustments for $\mathrm{RHI}(\mathrm{n}=70)$ include age, sex, race, $\mathrm{BMI}$, diabetes, and daily $\mathrm{PM}_{2.5}$. Asterisks indicate $\mathrm{p}$-values $<0.05$. Model estimates can be found in supplemental information. 
Figure 3-3. Percent differences and 95\% confidence intervals in catecholamines per IQR of VOC metabolite in the total cohort (+). Models were stratified by male participants (filled circle), female participants (open circle), Black participants (fliled triangle), and White participants (open triangle).
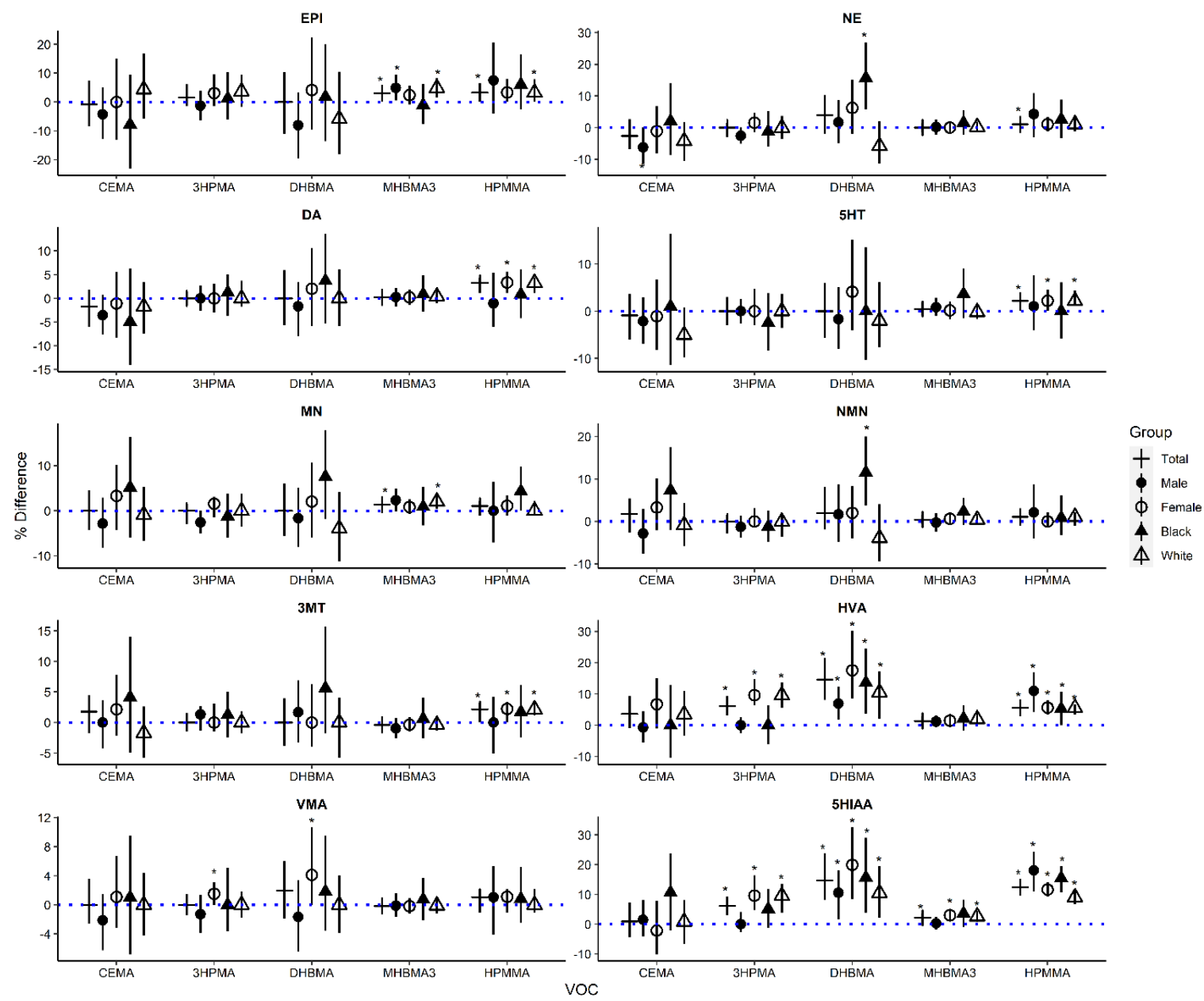

Catecholamines include epinephrine (EPI), norepinephrine (NE), dopamine (DA), 5hydroxythreonine (5HT), metanephrine (MN), normetanephrine (NMN), 3-methoxytyramine (3MT), homovanillic acid (HVA), vanillymandelic acid (VMA), and 5-hydroxyindole acetic acid $(5 \mathrm{HIAA})$. Asterisks indicate $p$-values $<0.05$. Model estimates can be found in supplemental information. 
levels of EPI tertiles (between 6.6 and $31.5 \mathrm{ng} / \mathrm{mg}$ ). There was a positive association between HPMMA and SBP (3.3\% higher; 95\% Cl: $0.7,5.9 \%)$ in participants with lower levels of EPI (between 0.0 and $3.1 \mathrm{ng} / \mathrm{mg}$ ). Similarly, there was a positive association between HPMMA and SBP $(2.7 \%$ higher; $95 \% \mathrm{Cl}: 0.0,5.4 \%)$ in participants with lower levels of DA tertiles (between 5.2 and $133.7 \mathrm{ng} / \mathrm{mg})$.

\section{DISCUSSION}

In this study of participants with moderate to high CVD risk, we found that metabolites of acrolein and 1,3-butadiene, (3HPMA and DHBMA, respectively), were significantly associated with SBP and endothelial dysfunction. We also found that Black participants may be more susceptible to the effects of acrolein (with respect to SBP) and they had higher levels of NE and NMN associated with 1,3-butadiene exposure. DHBMA was consistently associated with the dopamine metabolite homovanillic acid, across all strata. The crotonaldehyde metabolite, HPMMA, was strongly associated with the serotonin metabolite, 5-hydroxyindole acetic acid. Finally, we report null results of catecholamines mediating the relationship between VOC exposure and blood pressure.

Acrolein is a reactive unsaturated aldehyde known to be associated with CVD risk. ${ }^{25}$ Acrolein can be produced both endogenously from lipid peroxidation, and exogenously from combustion, chemical production, cigarette smoke, and e-cigarette vapors. ${ }^{147,152}$ In the city of Louisville, Jefferson County census tracts were estimated to have some of the highest ambient acrolein concentrations across the state of Kentucky in 2011 and 2014, with point and mobile source emissions (airport and heavy duty vehicles) as top contributors. ${ }^{153}$ In our analysis, we found that the acrolein metabolite, 3HPMA, was significantly associated with increased SBP in a dose-dependent manner.

Similar to our results, previous studies have shown that exposure to acrolein increases BP in both normotensive and hypertensive rats. ${ }^{154,155}$ Additionally, several experimental studies have shown that exposure to acrolein affects endothelial function by suppressing endothelial nitric oxide synthase activation, ${ }^{156}$ attenuating endothelial cell migration, ${ }^{157}$ blocking vascular endothelial growth factor, ${ }^{158}$ and reducing circulating levels of angiogenic cells. ${ }^{25}$ Specifically, an 
intact endothelium mediates dilation of the mesenteric bed in response to acrolein exposure; thus indicating a blunted response with increased sympathetic tone. ${ }^{159}$ Endothelial function was similarly impaired in tobacco smokers ${ }^{160-162}$ and e-cigarette users ${ }^{163-166}$-two sources of acrolein exposure in the general population. Finally, CVD risk markers, EPI and NE did not increase with respect to acrolein exposure in male Wistar and GK rats. ${ }^{167}$

The chemical - 1,3-butadiene is an alkene reported to have a possible epidemiological association with arteriosclerotic heart disease.$^{168-170}$ This chemical is primarily used to make synthetic rubber, but it is also produced from petroleum processing and combustion sources such as vehicle exhaust and cigarette smoke. ${ }^{171}$ Although our study did not show associations between 1,3-butadiene exposure and BP, one study found normotensive pregnant women had increased odds of high BP when exposed to ambient 1,3-butadiene two hours prior to admission to labor and delivery. ${ }^{18}$ Another cross-sectional study used principal component analysis of nonsmokers' personal exposures to ambient VOCs; the authors observed decreased endothelial function and increased DBP in relation to a 1,3-butadiene source in the Detroit Exposure and Aerosol Research Study (DEARS). No effect on SBP was seen. ${ }^{172}$ Similar to acrolein, several studies suggest 1,3-butadiene as a major cardiovascular risk driver in cigarette smoke. ${ }^{169,} 173$ However, from these data the singular contribution of 1,3-butadiene exposure to endothelial dysfunction in humans could not be ascertained, as in our study.

Crotonaldehyde is an unsaturated aldehyde and an atherogenic compound. ${ }^{174-176}$ Crotonaldehyde is produced endogenously from the metabolism of 1,3-butadiene and exogenously from combustion sources like vehicle exhaust and cigarette smoke..$^{177-179}$ Crotonaldehyde and acrolein did not seem to share similar adverse health effects in our study despite a potential shared mode of action. ${ }^{178}$ However, crotonaldehyde exposure was weakly associated with EPI, NE, DA, HVA, and $5 \mathrm{HT}$ and strongly associated with serotonin metabolite 5HIAA, but had little or no relationship with BP or endothelial function. Consistent with this observation, a recent study shows that chronic inhalation exposure of mice to crotonaldehyde led to decreases in SBP and DBP and enhanced endothelial function. ${ }^{180}$ Crotonaldehyde is a noncompetitive inhibitor of aldehyde dehydrogenases which have been suggested to be involved 
in DA metabolism, release, and synthesis. ${ }^{181-183}$ Furthermore, cigarette smoke, a major source of acrolein, 1,3-butadiene, and crotonaldehyde, has been shown to inhibit monoamine oxidase, a catalyst for the biotransformation of both DA and 5HT to metabolites HVA and 5HIAA, respectively. ${ }^{143}$ Therefore, our study suggests exposure to crotonaldehyde may affect dopaminergic pathways.

We found that Black participants may be more susceptible to acrolein exposure with respect to SBP, despite lower levels of exposure when compared with White participants. Black individuals are at an increased risk for $\mathrm{CVD}^{184}$ which may be explained by increased susceptibility to acrolein exposure, a major driver of CVD. ${ }^{185}$ Similar to our study, a previous analysis of NHANES 2005-2006 data reported Black individuals have lower urinary 3HPMA concentrations compared with White individuals. ${ }^{186}$ However, racial disparities in the relationship between acrolein and blood pressure has not previously been reported. This susceptibility may be explained by a myriad of factors such as access to healthcare or increased stress from systemic racism, and thus requires future research. In Louisville, Kentucky, the National Air Toxics Assessment estimates higher concentrations of acrolein in lower percent Black census tracts, but the health impact of such exposures has not been evaluated. ${ }^{153}$

Black individuals also had higher levels of NE and NMN associated with 1,3-butadiene exposure. Two large, retrospective epidemiological studies have reported increased standardized mortality ratios for arteriosclerotic heart disease in 1,3-butadiene exposed rubber plant workers, particularly in the Black male population. ${ }^{168-170}$ Similarly, another study found that Black hypertensive subjects had the more sensitive and highest-density $\beta$-receptors, ${ }^{187}$ and increased $\alpha 1$ and $\beta$-adrenergic receptor responsiveness compared with White subjects. ${ }^{188}$ Because EPI, $\mathrm{NE}$, and DA are agonists for $\beta$-adrenergic receptors, increased catecholamines could produce a higher $\beta$-adrenergic response in Black individuals leading to an increased risk for CVD. Additional studies with larger populations are needed to replicate these findings.

In our analyses, catecholamines did not mediate the relationship between VOC exposure and blood pressure. Mediation is the sum of the direct effect (c') and the indirect effect $(a \times b)$ of $X$ on $\mathrm{Y}$, the total effect. ${ }^{149}$ Although we found significant direct effects between the acrolein 
metabolites and catecholamines, the indirect effects were null. Therefore, we cannot conclude that catecholamines mediate the effects of VOC exposure on blood pressure in our study. These null effects may be explained by the small magnitude of change in catecholamines. Blood pressure medications, however, may be effect modifiers or confounders of these analyses because they reduce changes in catecholamines and may attenuate the mediating relationship between VOC exposures and blood pressure.

CVD is a complex group of pathological conditions that affect the heart and blood vessels. Although rare cases of CVD can be linked to specific gene-defects, a majority of CVD risk can be attributed to environmental factors such as poor nutrition, physical inactivity, smoking and exposure to air pollution. ${ }^{58,189,190}$ There are three proposed biological pathways through which exposure to air pollution could contribute to the development of CVD: 1) systemic oxidative stress and inflammation, 2) autonomic nervous system imbalance, and 3) transmission of constituents from the lung into the blood. ${ }^{6}$ We observed that exposure to 1,3-butadiene and crotonaldehyde were associated with endothelial dysfunction and biogenic monoamines, and that acrolein exposure was associated with SBP and endothelial dysfunction, suggesting that the risk of CVD attributable to the environment may be in part derived from exposure to VOCs that are ubiquitously present in the environment.

A major advantage of this study is that interindividual differences in exposure were accounted for by using human biomonitoring in the exposure assessment. Another advantage is the removal of smokers based on urinary cotinine values greater than $40 \mathrm{ng} / \mathrm{mg}$ of creatinine. Most studies of VOC exposure are unable to account for smoking, or they overestimate the contribution of smoking to VOC exposure. Here, we show how low levels of VOCs may contribute to CVD effects, and that these effects are independent of smoking. Although we chose to use urinary cotinine values greater than $40 \mathrm{ng} / \mathrm{mg}$ creatinine to remove smoking individuals, participants could still be exposed to low levels of VOCs from marijuana use, or intermittent smoking.

Limitations of this study include the small sample of nonsmokers, potential bias from cross-sectional study design and classification of nonsmokers, and exposure and outcome 
measurement error. Misclassification of exposure and outcome is an important source of bias in epidemiologic studies and is a likely limitation of our study. To quantify exposure, we measured urinary metabolites. However, urinary metabolites of VOCs range widely in half-life, and therefore their variable levels due to differences in times of exposure may account for some of the variability in the data and exposure misclassification. Finally, even though we tested multiple associations from individual VOC metabolites, we did not adjust for multiple comparisons which may have allowed for some spurious associations. ${ }^{150}$

Further work is required to corroborate the findings of our study. Data from larger cohorts with VOC metabolite data may be particularly informative about these associations in the general population. However, cohorts like NHANES do not have extensive data on CVD risk factors or markers of sub-clinical disease progression. While several cohorts such as the Multi-Ethnic Study of Atherosclerosis (MESA) or the Framingham Heart Study have rich data on CVD risk, progression, and outcomes, they do not have VOC exposure estimates. Nonetheless, characterization of VOC exposure in these cohorts may be useful in further strengthening the associations identified in our work. Additional work is also required to identify sources of exposure. Source apportionment based on area of residence could be useful in defining geographic exposure contributors.

Approximately $70 \%$ of noncommunicable diseases like CVD are attributable to air pollution, the fifth leading human health risk factor. ${ }^{191}$ Although the effects of $\mathrm{PM}_{2.5}$ exposure on CVD are well documented in the literature, there is a lack of data regarding VOC exposures, which are a major component of air pollution, tobacco smoke, consumer products, and Superfund site generated pollutants. This gap is most likely attributable to the gap in VOC emission inventories and quantification of VOCs in ambient air, ${ }^{11,13-15}$ as well as the lack of measurements of VOC metabolites in exposed populations. Recently, volatile chemical products such as pesticides, coatings, printing inks, adhesives, cleaning agents, and personal care projects were reported to make up half of fossil fuel VOC emissions in industrialized cities. ${ }^{8}$ Our findings suggest that low levels of VOCs can contribute to CVD at least in an at-risk population, ${ }^{16,145}$ and highlight the importance of investigating VOC exposure as a risk factor in the development of 
CVD. Finally, our findings highlight the need to further survey, reduce, and regulate environmental pollutants for the prevention of CVD. 


\section{CHAPTER 4 POLLUTION MIXTURES ARE ASSOCIATED WITH CARDIOVASCULAR MORTALITY AND BLOOD PRESSURE}

\section{INTRODUCTION}

More than $70 \%$ of diseases caused by environmental factors are non-communicable diseases, of which CVDs are a major component. ${ }^{2}$ Specifically, evidence supports the association between exposure to VOCs, ${ }^{18,20,25,26,75,77}$ exposure to metals, ${ }^{192,}, 193$ and exposure to ambient $\mathrm{PM}_{2.5}{ }^{6,194,195}$ in the development of CVD. However, it is well known that these exposures do not occur singularly, in a vacuum. Yet, epidemiological studies have historically assessed the relationship between one exposure and multiple health effects. As a result, the impact of multiple environmental exposures on health has not been well characterized. The National Institute of Environmental Health Science 2018-2023 strategic plan highlights this gap by listing the assessment of co-exposures as a primary goal for advancing environmental health sciences. ${ }^{196}$

Environmental exposure data consisting of multiple exposures present challenges of high dimensionality, multi-collinearity, and multiple comparisons in biostatistical analysis. Therefore, there are two major types of statistical methods used to overcome these challenges in the study of mixtures, unsupervised and supervised methods. ${ }^{197}$ Unsupervised methods commonly use dimension reduction techniques independent of the modelled outcome and include clustering, principal component analysis, and exploratory factor analysis. Supervised methods are informed by the outcome of interest and include the penalized regression, otherwise known as variable selection, the weighted quantile sum regression, and the Bayesian Kernel Machine Regression (BKMR). Due to the myriad methods of assessing mixtures, one must consider the research question of interest and the data to be analyzed prior to choosing a method to assess mixtures. 
Until now, this dissertation work has focused on single exposure models to assess the association with CVD mortality and the CVD risk factors in Chapters 2 and 3. Here, we will use the previously mentioned TRI and LHHS data to cross-sectionally assess mixture patterns in relationship to our CVD outcomes of CVD mortality and blood pressure. The TRI dataset is a longitudinal study of county level toxic releases from industries and age-adjusted all cause and CVD cause mortality between 2002 and 2012 . The LHHS dataset is a cross-sectional study of Louisville residents with moderate to high CVD risk. Between October 2009 and April 2017, participants were recruited at baseline at the University of Louisville Hospital and associated clinics. The entire cohort was comprised of 615 participants. The Institutional Review Board (IRB) at the University of Louisville approved the study.

As we are interested in how these environmental mixtures are associated with a cardiovascular outcome of mortality or blood pressure, we will be using two supervised methods, variable selection and BKMR. The variable selection procedure, elastic net, will be used to assess the relationship between mixtures of county level toxic releases and age-adjusted CVD mortality in 2012. The BKMR method will be used to assess the relationship between mixtures of urinary VOC metabolites and systolic and diastolic blood pressure (SBP and DBP). Each method will aid in the identification of potentially toxic mixture components.

\section{METHODS}

Study Populations, Exposures, and Outcomes

\section{National County Level TRI Study}

The National County Level TRI Study (NCLTS) is an ecological, longitudinal study designed to examine the relationship between county-level toxic releases and county-level, ageadjusted all cause and circulatory disease mortality, otherwise referred to as CVD mortality, between 2002 and 2012 in the contiguous United States. Exposure and outcome data was acquired from publicly available data from the EPA Toxic Release Inventory database and CDC Wonder database. County-level covariates were acquired from the U.S. Census Bureau, the U.S. Department of Labor, and the Institute for Health Metrics and Evaluation. The dataset includes 
2,599 counties in the contiguous United States with repeated observations for the consecutive years 2002 to 2012 , a total of 17,730 unique observations.

To assess county-level exposures, we acquired annual toxic chemical releases from the TRI. ${ }^{92}$ We downloaded national annual basic data files from the U.S. Environmental Protection Agency website for the consecutive years of 2002 to 2012. Section 313 of the Emergency Planning and Community Right to Know Act (EPCRA) promulgates the TRI. The TRI is a reporting system for facilities with a primary sector industry code included in the required reporting list, facilities employing ten or more people, or facilities manufacturing, processing or using EPCRA 313 chemicals. Facilities must report estimates of annual releases if they exceed thresholds of 25,000 pounds per year for manufacturing; 25,000 pounds per year for processing; or 10,000 pounds per year for otherwise use.

To assess mortality outcomes, we used data on deaths by underlying cause of death and county of residence from vital registration through the National Center for Health Statistics ${ }^{106}$ and on population data from the U.S. Census Bureau ${ }^{107}$ for intercensal estimates of county-level percentages of sex, and race. From 2002 to 2012, there were a total of 31.8 million deaths in the contiguous U.S.; 11 million of these deaths were from circulatory disease (ICD-10 codes beginning with I) for which there is evidence of an association with toxic chemical pollution. ${ }^{2}$ All exposure and outcome variables and covariates were harmonized and merged to create a dataset of publicly available data. All variables were matched by county-level FIPS codes, the Federal Information Processing Standard.

\section{Louisville Healthy Heart Study}

The Louisville Healthy Heart Study (LHHS) is a cross-sectional study designed to examine the relationship between exposure to environmental pollutants and CVD risk. The study recruited 615 participants with moderate to high CVD risk at University of Louisville Hospital and associated clinics in Louisville, KY between October 2009 and April 2017. Smoking is a known risk factor for CVD, and a major source and confounder of VOC exposure. ${ }^{138,145,147}$ To assess environmental VOC exposure in individuals, we restricted the cohort to nonsmokers. We 
excluded individuals who were smokers, classified as individuals with urinary cotinine levels greater than $40 \mathrm{ng} / \mathrm{mg}$ of creatinine $(\mathrm{n}=222)$, despite self-reported positive smoking status.

To assess VOC exposure, the levels of 22 urinary metabolites of 17 parent VOCs, (acrolein, acrylamide, acrylonitrile, benzene, 1-bromopropane, 1,3-butadiene, crotonaldehyde, $\mathrm{N}, \mathrm{N}$-dimethylformamide, ethylbenzene, ethylene oxide, propylene oxide, styrene, tetrachloroethylene, toluene, trichloroethylene, vinyl chloride, and xylene) were quantified using a modified version of the UPLC-MS method. ${ }^{86,145}$ In brief, urine was diluted with $15 \mathrm{mM}$ ammonium acetate and spiked with isotopically labeled internal standards. The analysis was performed on an Acquity UPLC core system coupled to a Quattro Premier XE triple quadrupole mass spectrometer with an electrospray source (Waters Inc, MA). ${ }^{145}$

The VOC metabolites were analyzed using a method adopted from CDC. ${ }^{86,139}$ The method was validated ${ }^{139}$ and the results showed that the sensitivity, precision, and accuracy of our method are comparable to the method reported. Specifically, the limit of detection (LOD) of each analyte is typically within 1 to $10 \mathrm{ng} / \mathrm{ml}$. Precision, assessed by the coefficient of variation of quality control samples, was within $17 \%$. Accuracy, assessed by analyzing spiked urine, was determined at three different levels of VOC metabolites, and was within 80 to $120 \%$. The method is highly reproducible, with relative standard deviations $<8 \%$. The sensitivity, accuracy, and precision were similar to those associated with the method developed by the CDC.

The primary exposure variables were 22 VOC urinary metabolites: N-Acetyl-S-(2carboxyethyl)-L-cysteine (CEMA), N-Acetyl-S-(3-hydroxypropyl)-L-cysteine (3HPMA), N-Acetyl-S(2-cyanoethyl)-L-cysteine (CYMA), N-Acetyl-S- (2-hydroxyethyl)-L-cysteine (HEMA), t,t-Muconic Acid (MU), N-Acetyl-S-(n-propyl)-L-cysteine (BPMA), N-Acetyl-S-(3,4-dihydroxybutyl)-L-cysteine (DHBMA), N-Acetyl-S-(4-hydroxy-2-buten-1-yl)-L-cysteine (MHBMA3), N-Acetyl-S-(3hydroxypropyl-1-methyl)-L-cysteine (HPMMA), N-Acetyl-S-(N-methylcarbamoyl)-L-cysteine (AMCC), Phenylglyoxylic acid (PGA), N-Acetyl-S-(2-hydroxypropyl)-L-cysteine (2HPMA), NAcetyl-S-(1-phenyl-2-hydroxyethyl)-L-cysteine + N-Acetyl-S-(2-phenyl-2-hydroxyethyl)-L-cysteine (PHEMA), Mandelic Acid (MA), N-Acetyl-S-(trichlorovinyl)-L-cysteine (TCVMA), N-Acetyl-S(benzyl)-L-cysteine (BMA), N-Acetyl-S-(1,2-dichlorovinyl)-L-cysteine (12DCVMA), N-Acetyl-S- 
(2,2-dichlorovinyl)-L-cysteine (22DCVMA), Urinary N-Acetyl-S-(dimethylphenyl)-L-cysteine (DPMA), 2-Methylhippuric acid (2MHA), and 3-Methylhippuric acid + 4-Methylhippuric acid $(3,4 \mathrm{MHA})$. Analytes were normalized to individual urinary creatinine levels to adjust for dilution, and reported as levels (nanograms of metabolite per milligram of creatinine, $\mathrm{ng} / \mathrm{mg}$ ).

Blood pressure was collected and measured at time of enrollment. Systolic and diastolic BP were measured after ten minutes of rest with an automated cuff and recorded as continuous variables. Three measurements were taken one minute apart with the last two measurements averaged.

\section{Statistical Analysis}

\section{Variable Selection}

In simple linear regression, we draw a line of best fit given data points $x$ and $y$ to summarize the relationship between $x$ and $y$. To find the beta estimate and the best fit line, we minimize the residual sum of squares, otherwise known as ordinary least squares (OLS). However, traditional OLS offers poor prediction accuracy and interpretation, due to low bias and large variance, and multiple predictors.

Penalization techniques have been proposed to improve OLS estimates. There are three types of penalization techniques: ridge regression, least absolute shrinkage and selection operator (lasso), and elastic net. Ridge regression is a continuous shrinkage method which minimizes the residual sum of squares subject to a bound on the $\mathrm{L}_{2}$-norm of the coefficients (the sum of the squared coefficients). ${ }^{198}$ This method improves prediction through a bias-variance trade-off, but it does not reduce the number of interpretable predictors. The lasso technique is a penalized least squares method imposing an $L_{1}$-penalty on the regression coefficients (the sum of the absolute coefficients). The lasso does both continuous shrinkage, as in ridge regression, and automatic variable selection, reducing the number of interpretable predictors. ${ }^{199}$ Limitations of the lasso are model saturation when there are more predictors than observations $(p>n)$, prediction performance domination by ridge regression when there are more observations than predictors $(n>p)$, and selection of one variable when predictor correlations are high. ${ }^{200}$ Elastic net has the 
advantage of simultaneously doing automatic variable selection, continuous shrinkage, and selection of groups of correlated variables. Elastic net constrains the model by shrinking regression coefficients of highly correlated variables towards each other, with a combination of the penalty terms L1 and L2 norm. ${ }^{200}$

If the dataset has $n$ observations and $p$ predictors, let $y=\left(y_{1} \ldots y_{n}\right)^{T}$ be the response and $X=\left(x_{1}|\ldots| x_{p}\right)$ be the model matrix, where $x_{j}=\left(x_{i j}, \ldots x_{n j}\right)^{T}, j=1, \ldots p$, are the predictors. For any fixed non-negative $\lambda_{1}$ and $\lambda_{2}$, we define the naïve elastic net criterion:

$$
L\left(\lambda_{1}, \lambda_{2}, \beta\right)=|y-X \beta|^{2}+\lambda_{2}|\beta|^{2}+\lambda_{1}|\beta|_{1}
$$$$
\text { where }|\beta|^{2}=\sum_{j=1}^{p} \beta_{j}^{2}, \beta_{1}=\sum_{j=1}^{p}\left|\beta_{j}\right| \text {. }
$$

The naïve elastic net estimator $\hat{\beta}$ is the minimizer of $\hat{\beta}=\operatorname{argmin}\left(\lambda_{1}, \lambda_{2}, \beta\right)$. This procedure can be viewed as a penalized least squares method. Let $\alpha=\lambda_{2} /\left(\lambda_{1}+\lambda_{2}\right)$; then solving beta hat is equivalent to the optimization problem $\hat{\beta}=\operatorname{argmin}|y-X \beta|^{2}$, subject to $(1-\alpha)|\beta|_{1}+$ $\alpha|\beta|^{2} \leq t$ for some $t$. We call the $(1-\alpha)|\beta|_{1}+\alpha|\beta|^{2}$ the elastic net penalty, which is a convex combination of the lasso and ridge penalty.

To begin, we subset the data to the year 2012 and summed the chemicals per FIPS codes based on total releases in pounds. Any chemicals made up of entirely zeroes were removed. We log transformed the outcome variable and predictor variables and any covariate county-level percentages were logit transformed. Then we created a training dataset and testing data set based on $80 \%$ of the data to build a predictive model, and $20 \%$ to evaluate the model. The $X$ variables included 467 chemicals (predictors/exposures), and 8 model sociodemographic and characteristic model adjustments, a possibility of $2^{467}=3.81 \mathrm{E}+140$ models. The GLMNET package in $\mathrm{R}$ fits the generalized linear model via penalized maximum likelihood. The algorithm is quite fast and can reduce quickly the number of $2^{p}$ models. ${ }^{201}$ For each variable selection method, we used the GLMNET procedure to fit the final model on the training data of the matrix of $X$ variables (the 467 chemicals and the 8 model adjustments). The 8 covariates were forced into the 
model by setting the penalty to zero so that model adjustments were not penalized. We tested the ridge regression model $(\alpha=0)$, the lasso model $(\alpha=1)$ and the elastic net model by using a tuning grid to determine the best $\alpha$ for the data. The $\alpha$ value controls the penalty and bridges the gap between ridge regression $(\alpha=0)$, elastic net ( $\alpha=$ between 0 and 1$)$, and lasso $(a=1)$. We found the best $\lambda$ by using the cross-validation technique. We use the minimum lambda to fit the model on the training data because it is the value of $\lambda$ at which the cross-validation takes the minimum mean squared error. Finally, we checked the prediction error for the test data to choose the best model.

\section{Bayesian Kernel Machine Regression}

Simple parametric models typically used in variable selection assume a simple functional form like a normal distribution. Semi- or nonparametric techniques are preferred in the identification of potentially synergistic effects of mixture components because they do not assume the functional form and allow adjustment for potential confounders. ${ }^{202}$ The Bayesian Kernel Machine Regression is a semi-parametric technique that models the exposure response relationship as a non-parametric kernel function of the mixture components, adjusting for covariates parametrically. The Gaussian kernel flexibly captures underlying functional forms without specifying the shape of the exposure-response curve. The BKMR method can assess independent effects, estimate the overall mixture, and assess for interactions among mixture components. ${ }^{203}$

Using BKMR, we can assume:

$$
Y_{i}=h\left(z_{i}\right)+x_{i}^{T} \beta+\epsilon_{i}
$$

Where $Y_{i}$ is a health endpoint, $z_{i}=\left(z_{i 1}, \ldots, z_{i M}\right)^{T}$ is a vector of $M$ exposure variables, $x_{i}$ contains a set of potential confounders, and $\epsilon_{i}{ }_{\sim}^{i . i . d} N\left(0, \sigma^{2}\right)$. In the context of mixtures, $h($.$) characterizes a$ high-dimensional exposure-response function that may incorporate non-linearity and interaction among the components of the mixture. Therefore, it is difficult to represent $h($.$) and is instead$ 
presented as a kernel machine representation. The Gaussian kernel is $K\left(z, z^{\prime}\right)=$ $\exp \left[-\sum_{m=1}^{M}\left(z_{m}-z^{\prime}{ }_{m}\right)^{2} / p\right]$ with $p$ tuning parameter. The Gaussian kernel flexibly captures a wide range of underlying functional forms for $h(.) .^{203}$

We created models for multiple VOC exposures. Results are reported for the following five VOC metabolites (CEMA, 3HPMA, DHBMA, MHBMA3, and HPMMA) of three parent compounds (acrolein, 1,3-butadiene, and crotonaldehyde). To assess multiple VOC models, we included VOC metabolites that showed statistically significant associations with vascular outcomes in single-VOC models in chapter 3 . We applied a BKMR approach to flexibly model the relationship between VOC metabolites CEMA, 3HPMA, DHBMA, MHBMA3, and HPMMA and blood pressure. The method also allowed us to examine statistical interactions between VOC metabolites within the mixture and joint associations between the whole mixture and blood pressure. The BKMR creates posterior inclusion probabilities (PIPS) that quantify the relative importance of each exposure in the model. ${ }^{203}$ Hierarchical variable selection is used when components of the mixture are highly correlated $(\rho>0.7)$. Because the VOC metabolites were not highly correlated (Figure 3), we did not use the hierarchical variable selection procedure of the BKMR.

All statistical analyses and visualizations were created with $R$ software (version 4.0.2). ${ }^{120}$ Additionally we used the tidyverse, ${ }^{119}$ glmnet, ${ }^{204}$ and kmbayes ${ }^{205}$ packages to analyze the data.

\section{RESULTS}

\section{Variable Selection}

County-level population estimates for year 2012 are reported in Table 4-1. There are currently 767 listed chemicals and 33 chemical categories covered under EPCRA Section 313. We removed $300 \mathrm{TRI}$ chemicals from analysis due to missing values, leaving a total of 467 predictors to be included in the matrix (Appendix A). The top released chemicals in pounds in 2012 include metal compounds lead, copper, arsenic, and zinc. Lead compounds were the most released compounds in 2012 at a total of 109 million pounds and qualify as the most released Clean Air Act chemical. 
For ridge regression, the best $\lambda$ value obtained from cross-validation is 11.2265 (Table 42). Using this $\lambda$ generated 446 out of 467 non-zero coefficients. The mean squared error was 0.0252 , the largest error of the three models.

Table 4-1. Descriptive statistics [mean ( \pm standard deviation (SD))] for county-level characteristics in the National County Level Study $(n=2,287)$ in 2012.

\begin{tabular}{|c|c|}
\hline Variable & Mean (SD) \\
\hline $\mathrm{n}$ & 2287 \\
\hline CVD Age Adj Rate ${ }^{a}$ & $251.61(59.20)$ \\
\hline$\%$ Male & $0.50(0.02)$ \\
\hline$\%$ White & $0.85(0.15)$ \\
\hline$\%$ Hispanic & $0.08(0.12)$ \\
\hline$\%$ Less than High School & $22.26(8.29)$ \\
\hline Median $\mathrm{HH}$ Income ${ }^{\mathrm{b}}$ & $37237.08(9462.44)$ \\
\hline Total Mean Smoking & $23.68(3.97)$ \\
\hline Alcohol & $50.09(11.34)$ \\
\hline $\mathrm{PM}_{2.5^{\mathrm{C}}}$ & $8.45(1.35)$ \\
\hline
\end{tabular}

${ }^{\text {a }}$ County level age-adjusted circulatory disease mortality rate in 2012

${ }^{b}$ County-level median household income in 2012

c County-level PM2.5 concentrations in 2012

Table 4-2. Mean squared errors (MSE) and tuning parameters of the three variable selection techniques, ridge regression, lasso, and elastic net. The ridge regression uses a tuning parameter of $\alpha=0$, lasso uses a tuning parameter of $\alpha=1$, and the elastic net procedure uses a tuning parameter of $\alpha=0.3$.

\begin{tabular}{cccccc}
\hline $\begin{array}{c}\text { Ridge } \\
\text { Lambda }\end{array}$ & $\begin{array}{c}\text { Ridge } \\
\text { MSE }\end{array}$ & Lasso Lambda & $\begin{array}{c}\text { Lasso } \\
\text { MSE }\end{array}$ & $\begin{array}{c}\text { Elastic Net } \\
\text { Lambda }\end{array}$ & $\begin{array}{c}\text { Elastic Net } \\
\text { MSE }\end{array}$ \\
\hline 11.2265 & 0.0252 & 0.0111 & 0.0245 & 0.0256 & 0.0244 \\
\hline
\end{tabular}

For lasso, the best $\lambda$ value obtained from cross-validation is 0.0111 . Using this $\lambda$ in the lasso model did not generate any non-zero coefficients for any TRI chemicals. County-level demographics and characteristics were forced into the model and thus were the only variables for which there were non-zero coefficients. 
With elastic net, we estimated $\alpha=0.3$ and $\lambda=0.0256$ from cross-validation. The elastic net model using these $\lambda$ and $\alpha$ values generated non-zero coefficients for five of the 467 TRI chemicals included (Figure 4-1). The five chemicals included were bromoform, dichlorobromomethane, dichlorotrifluoroethane, nitrophenol, and thallium. Given that the $\alpha=1$ for the lasso procedure, different non-zero coefficients are expected for an elastic net regularization with $\alpha=0.3$, an alpha closer to the ridge regression. The mean squared error was smaller for the elastic net procedure (Table 4-2) and is the preferred variable selection technique for the data.

Figure 4-1. Beta coefficients plotted for each selected variable in the elastic net regularization technique.

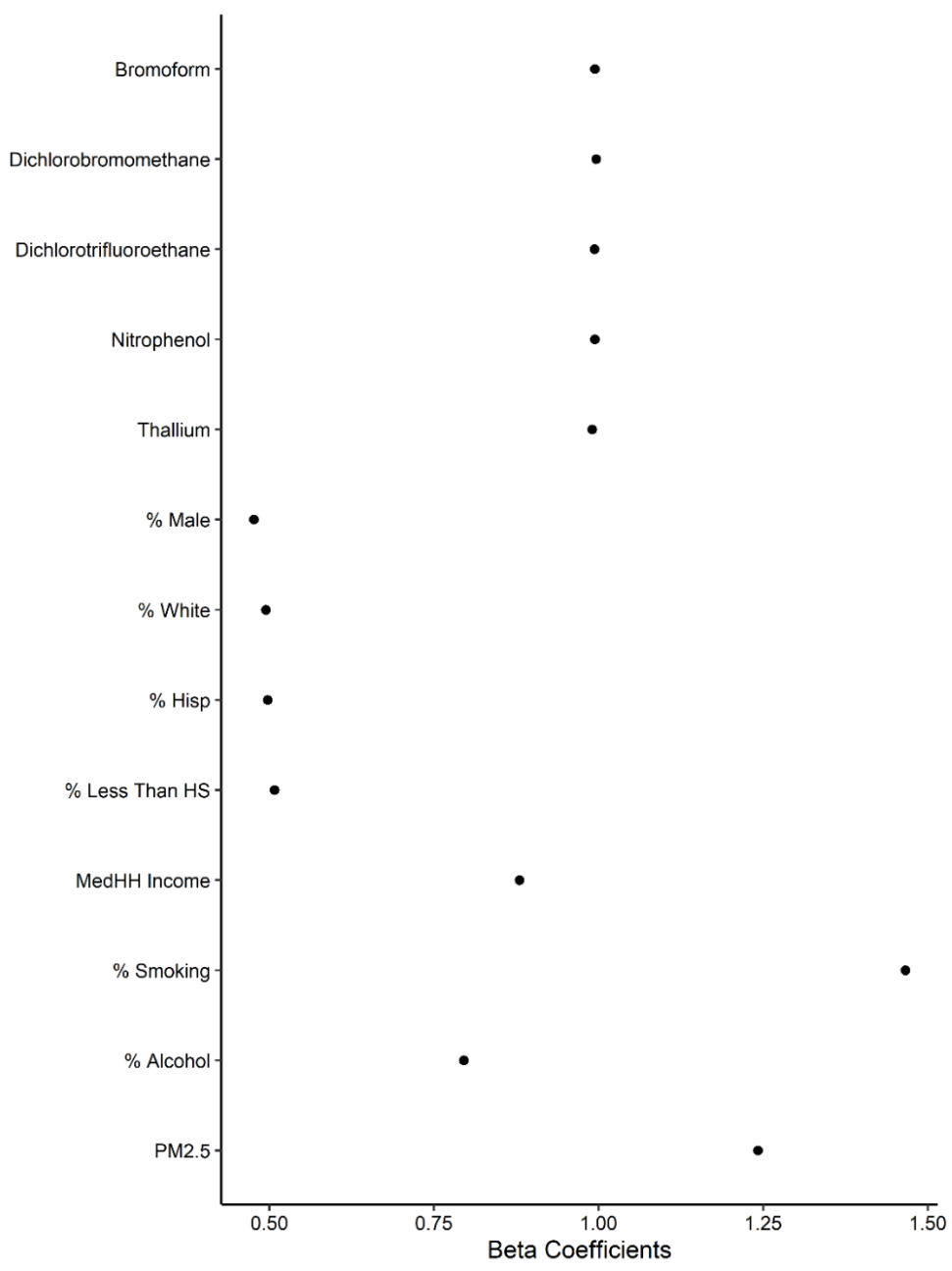




\section{Bayesian Kernel Machine Regression}

Because the VOC metabolites were not highly correlated (Figure 4-2), we did not use the hierarchical variable selection procedure of the BKMR. In the BKMR model, the metabolites 3HPMA and DHBMA had higher posterior inclusion probabilities (PIPs) for SBP and DBP, respectively (Table 4-3), indicating that 3HPMA and DBHMA were the most important metabolites of the group in relation to SBP and DBP. When VOC metabolite predictors were fixed to the $50^{\text {th }}$ percentile, the 3HPMA exhibited a linear relationship with SBP and several individual VOCs exhibited a linear relationship with DBP in univariate analysis (Figure 4-3 and 4-4). There was a positive linear relationship between the whole mixture and SBP, and between the whole mixture and DBP, when all other predictors were fixed at a range of percentiles $\left(25^{\text {th }}\right.$ to $75^{\text {th }}$ by 0.05$)$, compared with when they were fixed to the $50^{\text {th }}$ percentile (Figure 4-5 and 4-6). There was no evidence of interaction between VOC metabolites when all other exposures were fixed to a percentile or a second VOC was fixed to either the $25^{\text {th }}, 50^{\text {th }}$, or $75^{\text {th }}$ percentile (Figure 4-7 and 48). However, 3HPMA was a critical component of the mixture acting as the primary driver in the relationship with SBP.

Figure 4-2. Beta coefficients plotted for each selected variable in the elastic net regularization technique.

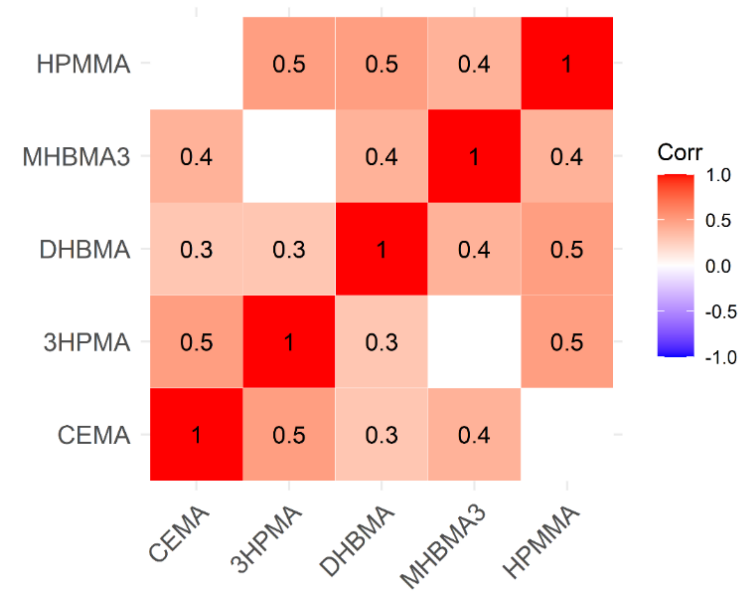


Table 4-3. Posterior inclusion probabilities of individual VOC metabolites in the Bayesian Kernel Machine Regression model.

\begin{tabular}{lcc}
\hline VOC $^{a}$ & SBP $^{a}$ & DBP $^{\mathrm{a}}$ \\
\hline CEMA & 0.0448 & 0.0412 \\
3HPMA & 0.1212 & 0.0128 \\
DHBMA & 0.099 & 0.0838 \\
MHBMA3 & 0.0462 & 0.0082 \\
HPMMA & 0.0786 & 0.0242 \\
\hline
\end{tabular}

aVolatile organic compound metabolite short name bSystolic blood pressure

'Diastolic blood pressure

Figure 4-3. Single-VOC metabolite exposure response association when all other metabolites are fixed to their 50th percentile.
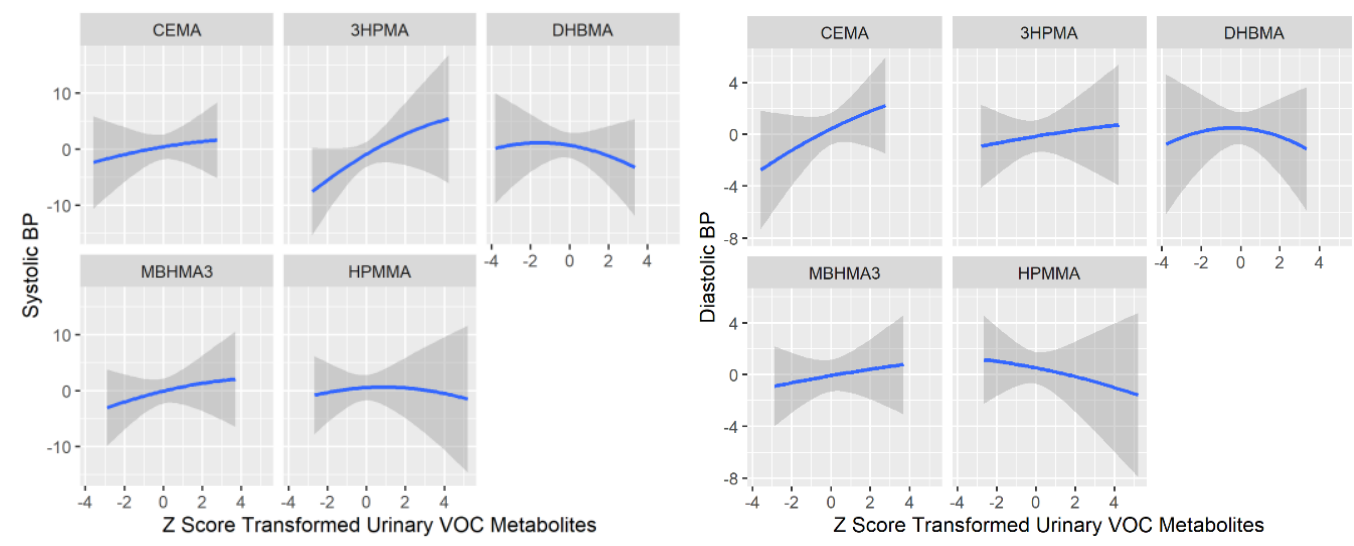

BKMR models adjusted for age, sex, race, BMI, ace inhibitor, ARBs, beta blockers, and daily PM2.5. 
Figure 4-4. Overall effect of the VOC mixture on systolic and diastolic blood pressure when all other predictors are at a particular percentile when compared with the value when all predictors are at their $50^{\text {th }}$ percentile.
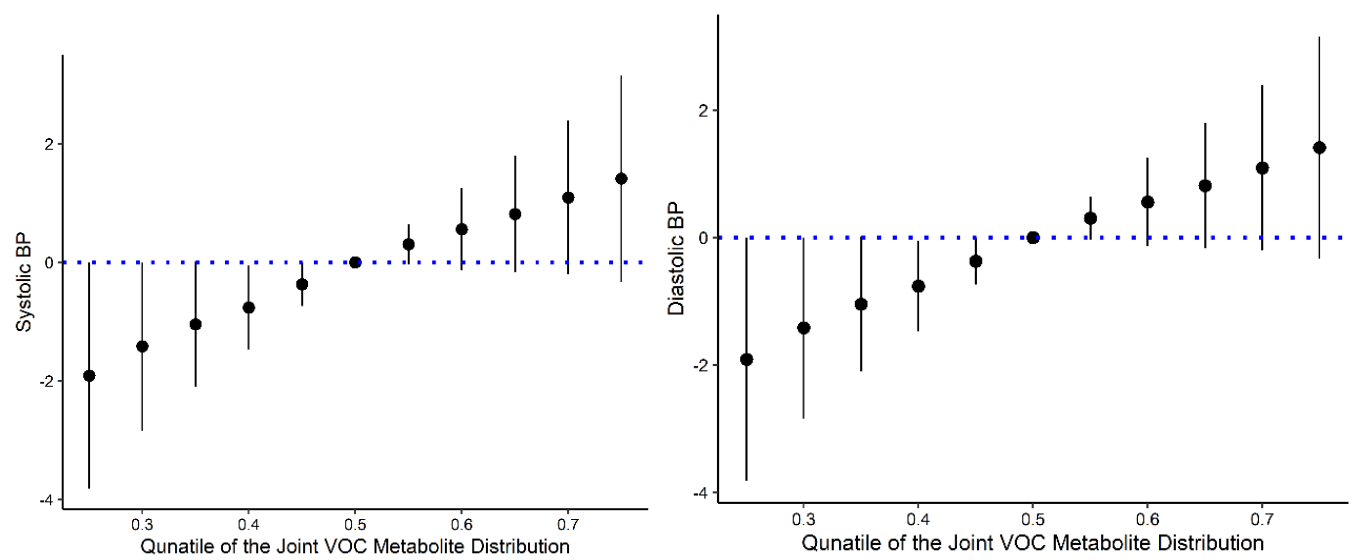

BKMR models were adjusted for age, sex, race, BMI, ace inhibitors, ARBs, beta blockers, and daily $\mathrm{PM}_{2.5}$.

Figure 4-5. Systolic and diastolic blood pressure for an interquartile range increase comparison of individual VOC metabolite concentrations when all other VOC metabolites have been fixed to the 25th, 50th, and 75th percentiles.
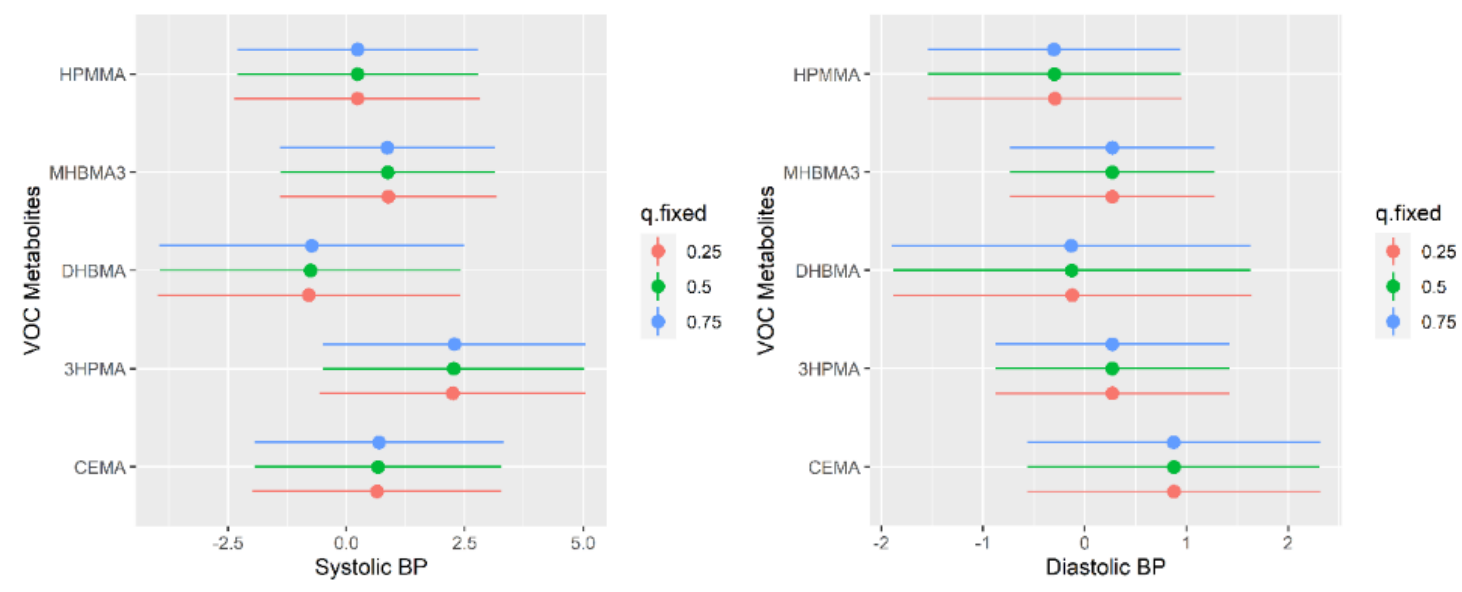


\section{DISCUSSION}

Using two supervised methods to assess mixtures, we were able to understand the scope of environmental exposures and how they are associated with CVD outcomes. Using the variable selection technique, elastic net, we identified five TRI chemicals bromoform, dichlorobromomethane, dichlorotrifluoroethane, nitrophenol, and thallium as the chemicals most strongly associated with increased county-level age-adjusted CVD mortality rates in 2012. Using the machine learning technique, BKMR, we identified 3HPMA and DHBMA as the most toxic VOC metabolites associated with SBP and DBP and found a significantly positive overall mixture effect, but no interactions.

The variable selection technique, elastic net, identified five chemicals most associated with CVD mortality, bromoform, dichlorobromomethane, dichlorotrifluoroethane, nitrophenol, and thallium. Bromoform and dichlorobromomethane are two trihalomethanes otherwise known as disinfection byproducts with some evidence of congenital anomalies. ${ }^{206-209}$ Dichlorotrifluoroethane is a hydrochlorofluorocarbon (HCFC) more commonly known as refrigerants like Freon.

Regulatory agencies have been phasing out HCFCs as part of the Montreal Protocol due to their ozone depleting nature. However, the extent to which HCFCs affect cardiovascular health is less studied. Nitrophenol is used in the production of drugs, fungicides, insecticides, and dyes. A recent longitudinal study of pesticide biomarkers found that type-1 diabetes diagnosed adolescents with high concentrations of nitrophenol had high $\mathrm{HbA1c}$ and low fasting C-peptide levels, two risk factors in the development of CVD. ${ }^{210}$ Thallium is a heavy metal produced from cigarette smoke, mine waste discharge, ore-processing, and coal-burning plants. The primary symptom of thallium poisoning in humans is hypertension. ${ }^{211}$

Bayesian Kernel Machine Regression revealed 3HPMA and DHBMA were the most important contributors of the mixture associated with SBP and DBP, respectively. The parent compounds, acrolein and 1,3-butadiene are known to be associated with CVD risk. ${ }^{25,}$ 168-170 Acrolein can be produced both endogenously from lipid peroxidation, and exogenously from combustion, chemical production, cigarette smoke, and e-cigarette vapors. ${ }^{147,152} 1,3$-Butadiene is primarily used to make synthetic rubber, but it is also produced from petroleum processing and 
combustion sources such as vehicle exhaust and cigarette smoke. ${ }^{171}$ Similar to our results, previous studies have shown that exposure to acrolein increases BP in both normotensive and hypertensive rats. ${ }^{154,155}$ Another study found normotensive pregnant women had increased odds of high BP when exposed to ambient 1,3-butadiene two hours prior to admission to labor and delivery. ${ }^{18}$ Another cross-sectional study used principal component analysis of nonsmokers' personal exposures to ambient VOCs. The authors observed decreased endothelial function and increased DBP in relation to a 1,3-butadiene source in the Detroit Exposure and Aerosol Research Study (DEARS). No effect on SBP was seen. ${ }^{172}$

The advantages of this study are the use of methods to assess the association between mixtures of environmental exposures and CVD. These methods reduce high dimensionality, high correlation, and multiple comparisons in large datasets. Compared to unsupervised methods, supervised methods are advantageous due to the consideration of the outcome of interest in the model. Finally, supervised methods have better prediction accuracy than traditional ordinary least squares methods. In the National County Level TRI Study, we used three variable selection procedures, ridge regression, lasso, and elastic net, to determine the best fit model for the most toxic components associated with county-level age-adjusted mortality rates. Variable selection is a regularization technique used to penalize lesser contributing predictors in the model toward zero and select the most important predictors. Variable selection reduced a high dimension dataset of 467 toxic chemical predictors to five chemical predictors associated with CVD mortality, which created a more interpretable set of data. In the Louisville Healthy Heart Study, we used the Bayesian Kernel Machine Regression technique to flexibly model the effect of the VOC metabolite mixture on blood pressure. This technique allowed us to visualize the univariate and bivariate relationship of VOC metabolites and blood pressure, assess interactions between VOC metabolites, and model the effect of the whole mixture on blood pressure. Furthermore, this method served as a confirmatory analysis to our initial single-pollutant model in Chapter 3. Finally, to the best of our knowledge, elastic net and BKMR have not yet been used on TRI data or VOC metabolite data. 
The major disadvantages of this study are the cross-sectional nature of both datasets, and the aggregate units in our national county-level study. Both studies are observational studies and cannot prove causation. Because the national county-level study uses an aggregate exposure and outcome variable, we cannot extrapolate the results to the individual level. Furthermore, the ecological design introduces ecological bias: bias due to interaggregate variation in the disease rate among those not exposed to the risk factor, bias due to groups acting as effect modifiers in the dose response relationship, and bias due to confounding variables. Finally, exposure misclassification may be another important source of bias in both datasets. In our use of variable selection to assess the National County Level TRI data, we are unable to estimate confidence intervals for nonzero coefficients, a major limitation of prediction. Additionally, estimated coefficients depend on the scaling of the predictors and may hinder interpretability. In our use of BKMR to assess the Louisville Healthy Heart data, we had a small dataset. The BKMR needs a large dataset due to the non-parametric kernel function. The kernel estimates the mixture-response relationship with a smoothing function and reduces the power. Finally, because measurement error varies across datasets, a statistical method will choose a chemical with less error over a more toxic chemical, as long as the two are correlated. ${ }^{212,213}$ Human populations are exposed to many anthropogenic pollutants which remain as complex mixtures in the environment. ${ }^{6,33}$ Although there is growing analysis of mixtures and copollutants in epidemiology studies, large gaps in mixture assessment persist. This study used two supervised methods, elastic net and BKMR, to determine the relevance of mixture components and the whole mixture in CVD outcomes. In our large matrix of $467 \mathrm{TRI}$ chemicals, the elastic net procedure identified five potentially toxic chemicals associated with county-level CVD mortality in 2012. In our smaller mixture of five VOC metabolites, the BKMR identified two metabolites as the most toxic VOC metabolites associated with blood pressure. This study implicates further study of the toxic compounds identified, acrolein, bromoform, 1,3-butadiene, dichlorobromomethane, dichlorotrifluoroethane, nitrophenol, and thallium and their role in the development of CVD. This study also highlights the importance of applying novel, supervised mixture assessment methods to a range of environmental exposure data. Finally, we want to emphasize the public health 
implications of using these methods to determine and better understand less studied pollutants and their contribution to adverse health effects. 


\section{CHAPTER 5 SUMMARY AND CONCLUSIONS}

\section{SUMMARY OF STUDY}

Overall, this work aimed to examine the effect of pollutants with lesser documented effects on cardiovascular disease using a multi-pronged approach to exposure assessment. The three aims were to examine the relationship between county-level toxic chemical releases and CVD mortality in the contiguous United States between 2002 and 2012, to assess the relationship between individual-level VOC metabolites and vascular function, and to build multipollutant models from the previous two aims to assess the role of mixtures and mixture components in CVD mortality and vascular function. Cardiovascular disease is the leading cause of death from environmental exposures. ${ }^{1}$ Because criteria pollutants require heavy surveillance under the Clean Air Act, evidence linking criteria pollutants and CVD preclude investigations of under regulated pollutants like TRI chemicals and VOCs. The significance of this work is outlined by new, longitudinal observations using publicly available TRI data, the elucidation of the mechanism of gaseous pollutants on vascular function, and the identification of potential toxic contributors to CVD. Furthermore, this work implicates the need for new environmental policies to regulate, surveil, and reduce pollutants in addition to the six criteria pollutants.

\section{MAJOR FINDINGS}

\section{National County Level TRI Study}

Exposure to environmental pollution is widespread, threatens all routes of exposure, and thus is the largest cause of disease and premature death in the world. ${ }^{2}$ Non-communicable diseases like CVD have largely been underestimated in relation to pollution. Particularly, the effects of chemical pollution on human health are poorly defined due to poor knowledge of many chemicals in common use..$^{90,91,130-132}$ Furthermore, the number of chemicals in commerce continues to grow exponentially despite unstudied potential adverse health effects. ${ }^{90}$ Therefore, 
the National County Level TRI Study was designed to examine the effect of pollution from eligible industries on age-adjusted all cause and circulatory disease mortality rates at the county level between 2002 and 2012.

County-level age-adjusted all-cause mortality rates, circulatory disease mortality rates, and the total pollution by quantity and by risk declined between 2002 and 2012 . These declines indicate improvements in pollution reduction programs and public health prevention. Few studies have examined the effects of county-level toxic releases across the nation, but the declines in mortality previously seen during industrial shutdowns and post pollution reduction programs ${ }^{214}$ indicate the rationale for further study. As this decline in mortality is attributed to declines in pollution, longitudinal examination in the National County Level TRI Study could help clarify the benefits of further reduction. Additionally, the use of risk reporting in reporting toxic releases to the TRI could further reduce mortality rates. Our longitudinal analysis of changes in county-level quantity-based and risk-based pollution showed significant associations with changes in both age-adjusted all-cause and circulatory disease mortality. Although the association between the risk-based pollution measure and all-cause mortality was smaller than that for the quantity-based measure, the association for the risk measure was somewhat larger for circulatory disease mortality.

Additional nationwide, longitudinal studies of pollution are needed to further strengthen these findings. Moreover, large studies must be more spatially granular to better understand the true extent to which pollution exposure affects individuals. However, this study is the first of its kind to longitudinally assess county-level pollution using mixed effects models. Additionally, the ecological nature of the study further supports previous reports on the benefits of pollution reduction in reducing mortality.

\section{Louisville Healthy Heart Study}

Volatile organic compounds (VOCs) are another group of pervasive environmental pollutants classified by their low molecular weight and high vapor pressure. Exposure to VOCs has been found to be associated with health effects such as CVD, neurological effects, organ toxicity, and cancer. ${ }^{16,36}$ However, most epidemiological studies examine the effects of VOC 
exposure using indirect exposure assessment methods such as ambient air monitoring stations. The Louisville Healthy Heart Study was designed to examine the effect of environmental pollutants from human biomonitoring on cardiovascular health. This study focused on the individual effect of single VOC pollutants on cardiovascular effects such as blood pressure, endothelial function, and sympathetic response.

Nonsmoking participants with higher VOC metabolite concentrations were older and had poor cardiovascular health. Specifically, there were significant associations between the acrolein metabolite, 3HPMA, and systolic blood pressure and endothelial dysfunction, and between the 1,3-butadiene metabolite, DHBMA, and endothelial dysfunction. Black participants had a larger association between 3HPMA and systolic blood pressure despite lower concentrations of 3HPMA. Several catecholamines were significantly associated with VOC metabolites. The crotonaldehyde metabolite, HPMMA, was weakly associated with epinephrine, norepinephrine, dopamine, homovanillic acid, and 5-hydroxytryptamine and strongly associated with serotonin metabolite 5-hydroxyindole acetic acid. These results seem to indicate that exposure to VOCs, acrolein, 1,3-butadiene, and crotonaldehyde play a mechanistic role in the development of CVD by increasing blood pressure, decreasing endothelial function, and increasing the sympathetic response.

Further work is required to corroborate the findings of our study. Data from larger cohorts with VOC metabolite concentrations at multiple timepoints may be particularly informative about these associations in the general population. However, this study lays the groundwork to begin to understand biological mechanisms that link air pollution constituents with cardiovascular disease, which can be used to help inform environmental health policy.

\section{Mixture Assessment}

Environmental exposures are multifactorial, heterogeneous mixtures. Observed adverse health effects associated with individual pollutants may be attributable to the combined effects of multiple pollutants. ${ }^{215}$ However, because environmental exposures are often highly correlated, conventional linear regression is complicated by issues of collinearity, high-dimensionality, and multiple comparisons. Therefore, the mixture assessment methods of the National County Level 
TRI Study and the Louisville Healthy Heart Study were designed to account for these issues in the data. We used three types of penalized regression, the ridge, lasso, and elastic net, to determine the potential toxic chemicals associated with circulatory disease mortality rates in the National County Level TRI Study. Additionally, we used the Bayesian Kernel Machine Regression to assess the exposure response function of the whole mixture of VOC metabolites and blood pressure in the Louisville Healthy Heart Study.

In the National County Level TRI Study, the best fit model used the elastic net variable selection procedure with an estimated $\alpha=0.3$ and $\lambda=0.0256$ from cross-validation. The elastic net model generated non-zero coefficients for five of the $467 \mathrm{TRI}$ chemicals included in the matrix. The five chemicals included were bromoform, dichlorobromomethane, dichlorotrifluoroethane, nitrophenol, and thallium. In the Louisville Healthy Heart Study, 3HPMA and DHBMA had higher posterior inclusion probabilities (PIPs) for systolic and diastolic blood pressure, respectively, indicating that 3HPMA and DBHMA were the most important metabolites of the group in relation to systolic and diastolic blood pressure. When VOC metabolite predictors were fixed to the $50^{\text {th }}$ percentile, the whole mixture exhibited a linear relationship with systolic and diastolic blood pressure. The acrolein metabolite, 3HPMA, was a critical component of the mixture acting as the primary driver in the relationship with SBP.

In general, more work is needed to understand the adverse health effects of pollutant mixtures and pollutant mixture components. The National County Level TRI Study analysis would be strengthened from a longitudinal penalized regression. Moreover, the large database of annual TRI emissions by chemical from 1979 to 2018 would be a great data source for further mixture assessment, particularly using penalized regression. Additionally, the Bayesian Kernel Machine Regression would be a useful method to apply to the wealth of urinary VOC metabolite data as well as other national biomonitoring studies.

\section{STRENGTHS}

There are several strengths of our multipronged approach to examine the effects of multiple pollutants on cardiovascular disease in human populations. Although ecological, the National County Level TRI Study modelled TRI chemicals and mortality rates at multiple 
timepoints using mixed models. Previous investigations have used averages to assess associations across multiple years using this data. ${ }^{19,104,105}$ In addition, we created a county-level risk score to better assess the toxicity of the chemicals released. Previously, studies have neglected to account for the TRI chemical risk level when modelling adverse health effects. ${ }^{97-99}$ Finally, to the best of our knowledge, supervised methods to assess mixtures have not yet been used to assess potential toxicity of TRI chemicals and their effect on cardiovascular health outcomes. Penalized regression methods reduced a high dimension dataset of 467 toxic chemical predictors to five chemical predictors associated with CVD mortality, which created a more interpretable set of data.

The Louisville Healthy Heart Study investigation was strengthened by a strong exposure assessment method and cotinine cutoff to remove confounding of VOC exposures from smoke exposure. ${ }^{23,138,216}$ Most studies of VOC exposure are unable to account for smoking, or they overestimate the contribution of smoking to VOC exposure. $64,65,172$ In addition, about two-thirds of the cohort reported a diagnosis of some type of CVD. As the prevalence of CVD continues to increase, it is important to capture susceptible individuals to provide accurate data for the prevention of CVD. Finally, our supervised methods were advantageous due to the consideration of the outcome of interest in the model and the better prediction accuracy than traditional ordinary least squares methods. We used the Bayesian Kernel Machine Regression technique to flexibly model the effect of the VOC metabolite mixture on blood pressure. This technique allowed us to visualize the univariate and bivariate relationship of VOC metabolites and blood pressure, assess interactions between VOC metabolites, and model the effect of the whole mixture on blood pressure.

\section{LIMITATIONS}

Limitations of this study include potential bias from cross-sectional and ecological study designs, and exposure and outcome measurement error. Misclassification of exposure and outcome is an important source of bias in epidemiologic studies and present limitations in our study. Ecological study designs, while beneficial, are aggregated data which cannot be extrapolated to the individual. Cross-sectional designs only provide one time point, muddying the 
inference for chronic diseases like CVD. Finally, in our method of modelling exposure to mixtures, we only assume exposure to the chemicals for which we have data. We do not consider the myriad other exposures present in the environment.

Although our ecological National County Level TRI Study is longitudinal, it is an observational study and cannot prove causation. Second, because the study uses an aggregate exposure and outcome variable, we cannot infer results to the individual level. Furthermore, the ecological design introduces ecological bias: bias due to interaggregate variation in the disease rate among those not exposed to the risk factor, bias due to groups acting as effect modifiers in the dose response relationship, and bias due to confounding variables. Finally, even though our study accounted for chemical toxicity, the values used were already established by the Agency for Toxic Substances and Disease Registry. While advantageous to our development of a risk score, reportable quantities are often based on limited or dated toxicology studies and do not represent more sensitive cardiovascular endpoints.

Limitations of the Louisville Healthy Heart Study include the small sample of nonsmokers, potential bias from cross-sectional study design and potential misclassification of nonsmokers, and exposure and outcome measurement error. Misclassification of exposure and outcome is an important source of bias in epidemiologic studies and is a likely limitation of our study. To quantify exposure, we measured urinary metabolites. However, urinary metabolites of VOCs range widely in half-life, and therefore their variable levels due to differences in times of exposure may account for some of the variability in the data and exposure misclassification. Finally, even though we tested multiple associations from individual VOC metabolites, we did not adjust for multiple comparisons which may have allowed for some spurious associations. ${ }^{150}$

The major limitations of assessing mixtures in the National County Level TRI Study and the Louisville Healthy Heart Study are the cross-sectional nature of both datasets, and the aggregate units. Although these methods were better predictive models, we could only predict at a cross-section of time, further limiting the strength of associations. In our use of variable selection to assess the National County Level TRI data, we are unable to estimate confidence intervals for nonzero coefficients, a major limitation of prediction. Additionally, estimated 
coefficients depend on the scaling of the predictors and may hinder interpretability. In our use of BKMR to assess the Louisville Healthy Heart data, we had a small dataset. The BKMR needs a large dataset due to the non-parametric kernel function. The kernel estimates the mixtureresponse relationship with a smoothing function and reduces the power. Finally, because measurement error varies across datasets, a statistical method will choose a chemical with less error over a more toxic chemical, as long as the two are correlated. ${ }^{212,213}$

\section{FUTURE DIRECTIONS}

More research is needed to explore less understood pollutants like TRI chemicals and VOCs. The TRI database is an under-utilized source of toxic chemical data dating back to 1987. Although the database tracks 737 eligible chemicals, it is an untapped resource of chemicals polluting our environment, many of which are not regulated. More work needs to be done to understand the role of specific chemicals and their toxicity in the development of cardiovascular

disease. Additionally, all chemical data have geographic coordinates and have the potential to be significant spatial data for adverse health outcomes at the individual level. Therefore, future studies must be designed to better understand chemical specificity and chemical toxicity using spatial granularity.

There is also a wealth of VOC metabolite data in several national cohorts, as well as the potential to measure these data in cohorts like the Multi-Ethnic Study of Atherosclerosis or the Framingham Heart Study. As the measurement of VOCs in human tissue is a sensitive exposure assessment method, this wealth of data must be used to better understand the mechanistic role of toxic VOC metabolites in the development of cardiovascular disease. Analyzing these cohorts will further strengthen the associations identified in our work by providing more statistical power.

More work must be done to understand the role of mixtures in environmental exposure data. Innovative statistical methods have been developed to investigate the role of multiple pollutants and must be used in addition to traditional single pollutant models. As environmental policy is governed by single pollutants, additional work on multiple pollutants will move environmental policy forward to better regulate multiple pollutants and reduce real-world exposures. Furthermore, there is a gap in risk reporting of known toxic chemicals, specifically to 
the TRI, as well as an increasing amount of untested chemicals. We want to emphasize the public health implications of better understanding less studied pollutants and their contribution to adverse health effects.

\section{CONCLUSIONS}

Overall, the work described here demonstrates the adverse cardiovascular health effects induced by exposure to pollutants and supports the hypothesis that exposure to pollutants such as TRI chemicals and VOCs contribute to the development of and mortality from cardiovascular disease, particularly through increased blood pressure, endothelial dysfunction, and increased sympathetic response. The aim of this work was to employ three distinct methods of exposure assessment to evaluate the relationship between exposure to pollutants and CVD. These methods include the indirect measure of source data from the Toxic Release Inventory, a direct measure of human biomonitoring, and the assessment of multiple pollutants using the novel statistical methods elastic net and Bayesian Kernel Machine Regression. Although the results of these methods were not directly comparable, they contribute novel assessments of environmental data to the field of environmental cardiology. These results elucidate the potential mechanisms of environmental pollutants on the development of CVD and identify potential contributors to CVD mortality. These results further implicate the need for improved environmental policy to prevent and reduce the prevalence of CVD. 


\section{REFERENCES}

1. Bhatnagar A. Environmental Determinants of Cardiovascular Disease. Circ Res. Jul 7 2017;121(2):162-180.

2. Landrigan PJ, Fuller R, Acosta NJR, et al. The Lancet Commission on pollution and health. Lancet. Feb 3 2018;391(10119):462-512.

3. World Health Organization W. Paper presented at: First WHO Global Conference on Air Pollution and Health; 30 October- 1 November 2018, 2018; Geneva, Switzerland.

4. Cohen AJ, Brauer M, Burnett R, et al. Estimates and 25-year trends of the global burden of disease attributable to ambient air pollution: an analysis of data from the Global Burden of Diseases Study 2015. Lancet. May 13 2017;389(10082):1907-1918.

5. Brook RD, Franklin B, Cascio W, et al. Air Pollution and Cardiovascular Disease. $A$ Statement for Healthcare Professionals From the Expert Panel on Population and Prevention Science of the American Heart Association. 2004;109(21):2655-2671.

6. Brook RD, Rajagopalan S, Pope CA, et al. Particulate matter air pollution and cardiovascular disease: an update to the scientific statement from the American Heart Association. Circulation. 2010;121.

7. Pope CA, 3rd, Turner MC, Burnett RT, et al. Relationships between fine particulate air pollution, cardiometabolic disorders, and cardiovascular mortality. Circ Res. Jan 2 2015;116(1):108-115.

8. McDonald BC, de Gouw JA, Gilman JB, et al. Volatile chemical products emerging as largest petrochemical source of urban organic emissions. Science. Feb 16 2018;359(6377):760-764.

9. Jerrett M, Burnett RT, Pope CA, et al. Long-Term Ozone Exposure and Mortality. New England Journal of Medicine. 2009;360(11):1085-1095.

10. Jimenez JL, Canagaratna MR, Donahue NM, et al. Evolution of Organic Aerosols in the Atmosphere. Science. 2009;326(5959):1525.

11. Strum M, Scheffe R. National review of ambient air toxics observations. Journal of the Air \& Waste Management Association. 2016/02/01 2016;66(2):120-133.

12. Weitekamp Chelsea A, Lein M, Strum M, et al. An Examination of National Cancer Risk Based on Monitored Hazardous Air Pollutants. Environmental Health Perspectives.129(3):037008.

13. Villeneuve PJ, Jerrett $\mathrm{M}, \mathrm{Su}$ J, et al. A cohort study of intra-urban variations in volatile organic compounds and mortality, Toronto, Canada. Environmental Pollution. 12// 2013;183:30-39.

14. Hoek $\mathrm{G}$, Beelen $\mathrm{R}$, de Hoogh $\mathrm{K}$, et al. A review of land-use regression models to assess spatial variation of outdoor air pollution. Atmospheric Environment. 2008/10/01/ 2008;42(33):7561-7578.

15. Wang L, Allen DT, McDonald-Buller EC. Air quality modeling of interpollutant trading for ozone precursors in an urban area. J Air Waste Manag Assoc. Oct 2005;55(10):15431557.

16. Konkle SL, Zierold KM, Taylor KC, Riggs DW, Bhatnagar A. National secular trends in ambient air volatile organic compound levels and biomarkers of exposure in the United States. Environ Res. Dec 2 2019;182:108991.

17. Ran J, Qiu H, Sun S, Yang A, Tian L. Are ambient volatile organic compounds environmental stressors for heart failure? Environmental Pollution. 2018/11/01/ 2018;242:1810-1816.

18. Mannisto T, Mendola $\mathrm{P}$, Laughon Grantz K, et al. Acute and recent air pollution exposure and cardiovascular events at labour and delivery. Heart. Sep 2015;101(18):1491-1498. 
19. Hendryx M, Luo J, Chen BC. Total and cardiovascular mortality rates in relation to discharges from Toxics Release Inventory sites in the United States. Environ Res. Aug 2014;133:36-41.

20. Villeneuve PJ, Jerrett $\mathrm{M}$, Su J, et al. A cohort study of intra-urban variations in volatile organic compounds and mortality, Toronto, Canada. Environ Pollut. Dec 2013;183:30-39.

21. Jung CC, Su HJ, Liang HH. Association between indoor air pollutant exposure and blood pressure and heart rate in subjects according to body mass index. Sci Total Environ. Jan 01 2016;539:271-276.

22. Xu X, Freeman NC, Dailey AB, llacqua VA, Kearney GD, Talbott EO. Association between exposure to alkylbenzenes and cardiovascular disease among National Health and Nutrition Examination Survey (NHANES) participants. Int J Occup Environ Health. Oct-Dec 2009;15(4):385-391.

23. Chambers DM, Ocariz JM, McGuirk MF, Blount BC. Impact of cigarette smoking on volatile organic compound (VOC) blood levels in the U.S. population: NHANES 20032004. Environ Int. Nov 2011;37(8):1321-1328.

24. Pazo DY, Moliere F, Sampson MM, et al. Mainstream Smoke Levels of Volatile Organic Compounds in 50 U.S. Domestic Cigarette Brands Smoked With the ISO and Canadian Intense Protocols. Nicotine \& Tobacco Research. 2016;18(9):1886-1894.

25. DeJarnett N, Conklin DJ, Riggs DW, et al. Acrolein exposure is associated with increased cardiovascular disease risk. J Am Heart Assoc. Aug 06 2014;3(4).

26. Abplanalp W, DeJarnett N, Riggs DW, et al. Benzene exposure is associated with cardiovascular disease risk. PLoS One. 2017;12(9):e0183602.

27. Jain RB. Distributions of selected urinary metabolites of volatile organic compounds by age, gender, race/ethnicity, and smoking status in a representative sample of U.S. adults. Environ Toxicol Pharmacol. Sep 2015;40(2):471-479.

28. Jain RB. Levels of selected urinary metabolites of volatile organic compounds in a representative sample of US adolescents. Toxicological \& Environmental Chemistry. 2016/09/13 2016;98(8):977-990.

29. U.S. Department of Health and Human Services. The Health Consequences of Smoking50 Years of Progress: A Report of the Surgeon General 2014.

30. U.S. Environmental Protection Agency. 2014 National Emissions Inventory (NEI) Data. 10/10/19. Available at: https://www.epa.gov/air-emissions-inventories/2014-nationalemissions-inventory-nei-data. Accessed 12/18/19, 2019.

31. United States Environmental Protection Agency U. Volatile Organic Compounds Emissions.

32. Definitions. 40 CFR § 51.100.

33. Lybarger JA, Lee R, Vogt DP, Perhac RM, Jr., Spengler RF, Brown DR. Medical costs and lost productivity from health conditions at volatile organic compound-contaminated superfund sites. Environ Res. Oct 1998;79(1):9-19.

34. Burg JR, Gist GL. Health effects of environmental contaminant exposure: an intrafile comparison of the Trichloroethylene Subregistry. Arch Environ Health. Jul-Aug 1999;54(4):231-241.

35. U.S. Environmental Protection Agency. Technical Overview of Volatile Organic Compounds. 4/12/17. Accessed 5/13/2019, 2019.

36. United States Environmental Protection Agency U. Hazardous Air Pollutants. 17 March 2017. Available at: https://www.epa.gov/haps.

37. Public Health and Welfare; 42 U.S.C. $\$ 7401$,.

38. Protection of the Environment; 40 C.F.R. $\S 50$

39. Office of Air Quality Planning and Standards. Ambient Air Monitoring Strategy for State, Local, and Tribal Air Agencies. In: EPA, ed. Research Triangle Park, NC: EPA; 2008:82.

40. U.S. Environmental Protection Agency. Managing Air Quality--Ambient Air Monitoring. 8/28/2018. Available at: https://www.epa.gov/air-quality-management-process/managingair-quality-ambient-air-monitoring. Accessed 5/30/2019, 2019.

41. Thacker SB, Stroup DF. Future directions for comprehensive public health surveillance and health information systems in the United States. Am J Epidemiol. Sep 1 1994;140(5):383-397. 
42. Morgan GB, Ozolins G, Tabor EC. Air Pollution Surveillance Systems. Science. 1970;170(3955):289.

43. U.S. Environmental Protection Agency. Air Toxics Data Analysis Workbook. In: Standards OoAQPa, ed. Research Triangle Park, NC; 2009:360.

44. U.S. Environmental Protection Agency. Air Toxics - National Air Toxics Trends Stations. 6/20/2019. Available at: https://www3.epa.gov/ttn/amtic/natts.html. Accessed 11/30/2019, 2019.

45. U.S. Environmental Protection Agency. Urban Air Toxics. 8/20/2019. Available at: https://www.epa.gov/urban-air-toxics. Accessed 11/30/2019, 2019.

46. U.S. Environmental Protection Agency. Fact Sheet: National Air Toxics Program Integrated Urban Strategy. Washington D.C.; 1999.

47. U.S. Environmental Protection Agency. Photochemical Assessment Monitoring Stations (PAMS). 9/29/2016. Available at: https://www3.epa.gov/ttn/amtic/pamsmain.html. Accessed 11/30/2019, 2019.

48. U.S. Environmental Protection Agency. Air Toxics - Community Scale Air Toxics Ambient Monitoring (CSATAM) Projects. 9/29/2016. Available at:

https://www3.epa.gov/ttn/amtic/local.html. Accessed 11/30/2019, 2019.

49. Srivastava A, Mazumdar, D.,. Air Quality Monitoring Assessment and Management. India: IntechOpen; 2011.

50. Libretexts. Gas Chromatography. 5/10/19. Available at:

https://chem.libretexts.org/Bookshelves/Analytical Chemistry/Supplemental Modules (A nalytical Chemistry)/Instrumental Analysis/Chromatography/Gas Chromatography.

Accessed 5/15/19, 2019.

51. Army Public Health Center. An Introduction to Environmental Health Risk Assessment. 7/27/2018. Available at:

https://phc.amedd.army.mil/topics/envirohealth/hrasm/Pages/Environment-Health-RiskAssessment.aspx. Accessed 11/30/2019, 2019.

52. United States Environmental Protection Agency U. Human Health Risk Assessment. 19 January 2017. Available at: https://www.epa.gov/risk/human-health-risk-assessment. Accessed 27 November 2017.

53. Abelsohn A, Frank J, Eyles J. Environmental public health tracking/surveillance in Canada: a commentary. Healthcare policy = Politiques de sante. 2009;4(3):37-52.

54. Agency for Toxic Substances and Disease Registry (ATSDR). Risk Assessment. Available at: https://www.atsdr.cdc.gov/sites/brownfields/pdfs/risk assessment-508.pdf. Accessed 11/30/2019, 2019.

55. Rothman KJ. Methodologic frontiers in environmental epidemiology. Environmental health perspectives. 1993;101 Suppl 4(Suppl 4):19-21.

56. World Health Organization W. Cardiovascular Diseases (CVDs). Accessed 22 November 2017.

57. Benjamin EJ, Blaha MJ, Chiuve SE, et al. Heart Disease and Stroke Statistics-2017 Update: A Report From the American Heart Association. Circulation. 2017.

58. Flowers E, Froelicher ES, Aouizerat BE. Gene-Environment Interactions in Cardiovascular Disease. European journal of cardiovascular nursing : journal of the Working Group on Cardiovascular Nursing of the European Society of Cardiology. 03/06 2012;11(4):472-478.

59. Kannel WB, Vasan RS. Adverse consequences of the 50\% misconception. Am J Cardiol. Feb 1 2009;103(3):426-427.

60. Shawky RM. Reduced penetrance in human inherited disease. Egyptian Journal of Medical Human Genetics. 2014/04/01/ 2014;15(2):103-111.

61. Brook RD, Rajagopalan S, Pope CA, 3rd, et al. Particulate matter air pollution and cardiovascular disease: An update to the scientific statement from the American Heart Association. Circulation. Jun 1 2010;121(21):2331-2378.

62. Kahn SE, Cooper ME, Del Prato S. Pathophysiology and treatment of type 2 diabetes: perspectives on the past, present, and future. The Lancet.383(9922):1068-1083.

63. HAWC: Health Assessment Workplace Collaborative [Computer software], [computer program]. Version; 2013. 
64. Nobles CJ, Williams A, Ouidir M, Sherman S, Mendola P. Differential Effect of Ambient Air Pollution Exposure on Risk of Gestational Hypertension and Preeclampsia. Hypertension. Aug 2019;74(2):384-390.

65. Zhu Y, Zhang C, Liu D, et al. Ambient Air Pollution and Risk of Gestational Hypertension. Am J Epidemiol. Aug 1 2017;186(3):334-343.

66. Chuang HC, Ho KF, Lin LY, et al. Long-term indoor air conditioner filtration and cardiovascular health: A randomized crossover intervention study. Environ Int. Sep 2017;106:91-96.

67. Jung CC, Su HJ, Liang HH. Association between indoor air pollutant exposure and blood pressure and heart rate in subjects according to body mass index. Sci Total Environ. Jan 1 2016;539:271-276.

68. Kotseva K, Popov T. Study of the cardiovascular effects of occupational exposure to organic solvents. Int Arch Occup Environ Health. Sep 1998;71 Suppl:S87-91.

69. Assadi SN. Electrocardiographic changes and exposure to solvents. J Arrhythm. Feb 2018;34(1):65-70.

70. Shin HH, Jones P, Brook R, Bard R, Oliver K, Williams R. Associations between personal exposures to VOCs and alterations in cardiovascular physiology: Detroit Exposure and Aerosol Research Study (DEARS). Atmospheric Environment. 2015/03/01/ 2015;104:246-255.

71. Kim JH, Moon JY, Park EY, Lee KH, Hong YC. Changes in oxidative stress biomarker and gene expression levels in workers exposed to volatile organic compounds. Ind Health. 2011;49(1):8-14.

72. Lin LY, Chuang HC, Liu IJ, Chen HW, Chuang KJ. Reducing indoor air pollution by air conditioning is associated with improvements in cardiovascular health among the general population. Sci Total Environ. Oct 1 2013;463-464:176-181.

73. Ha S, Mannisto T, Liu D, Sherman S, Ying Q, Mendola P. Air pollution and cardiovascular events at labor and delivery: a case-crossover analysis. Ann Epidemiol. Jun 2017;27(6):377-383.

74. Mannisto T, Mendola P, Liu D, Leishear K, Sherman S, Laughon SK. Acute air pollution exposure and blood pressure at delivery among women with and without hypertension. Am J Hypertens. Jan 2015;28(1):58-72.

75. Ran J, Qiu H, Sun S, Yang A, Tian L. Are ambient volatile organic compounds environmental stressors for heart failure? Environ Pollut. Nov 2018;242(Pt B):1810-1816.

76. Ye D, Klein $\mathrm{M}$, Chang HH, et al. Estimating Acute Cardiorespiratory Effects of Ambient Volatile Organic Compounds. Epidemiology. Mar 2017;28(2):197-206.

77. Ran J, Qiu H, Sun S, Tian L. Short-term effects of ambient benzene and TEX (toluene, ethylbenzene, and xylene combined) on cardiorespiratory mortality in Hong Kong.

Environment International. 2018/08/01/ 2018;117:91-98.

78. Tsai DH, Wang JL, Chuang KJ, Chan CC. Traffic-related air pollution and cardiovascular mortality in central Taiwan. Sci Total Environ. Mar 15 2010;408(8):1818-1823.

79. Arroyave WD, Mehta SS, Guha N, et al. Challenges and recommendations on the conduct of systematic reviews of observational epidemiologic studies in environmental and occupational health. Journal of Exposure Science \& Environmental Epidemiology. 2021/01/01 2021;31(1):21-30.

80. Harper M, Weis C, Pleil JD, et al. Commentary on the contributions and future role of occupational exposure science in a vision and strategy for the discipline of exposure science. Journal of Exposure Science \& Environmental Epidemiology. 2015/07/01 2015;25(4):381-387.

81. Jain RB. Detection rates, trends in and factors affecting observed levels of selected volatile organic compounds in blood among US adolescents and adults. Environ Toxicol Pharmacol. Dec 2017;56:21-28.

82. Su F-C, Mukherjee B, Batterman S. Determinants of personal, indoor and outdoor VOC concentrations: An analysis of the RIOPA data. Environmental Research. 2013/10/01/ 2013;126:192-203.

83. Council NR. Air Quality Management in the United States. Washington, DC: The National Academies Press; 2004. 
84. National Science and Technology Council. Air Quality Observation Systems in the United States. Washington DC, 2013.

85. Castell N, Dauge FR, Schneider P, et al. Can commercial low-cost sensor platforms contribute to air quality monitoring and exposure estimates? Environment International. 2017/02/01/ 2017;99:293-302.

86. Alwis KU, Blount BC, Britt AS, Patel D, Ashley DL. Simultaneous analysis of 28 urinary VOC metabolites using ultra high performance liquid chromatography coupled with electrospray ionization tandem mass spectrometry (UPLC-ESI/MSMS). Anal Chim Acta. Oct 31 2012;750:152-160.

87. Heinrich-Ramm R, Jakubowski, M., Heinzow, B., Christensen, J.M., Olsen, E., Hertel, O.,. Biological Monitoring for Volatile Organic Compounds (VOCs). Pure and Applied Chemistry. 2000;72(3):385-436.

88. World Health Organization (WHO). Noncommunicable Diseases. Available at: https://www.who.int/news-room/fact-sheets/detail/noncommunicable-diseases. Accessed 11/23/20, 2020.

89. Collaborators GRF. Global, regional, and national comparative risk assessment of 79 behavioural, environmental and occupational, and metabolic risks or clusters of risks, 1990-2015: a systematic analysis for the Global Burden of Disease Study 2015. Lancet (London, England). 2016;388(10053):1659.

90. Roundtable on Environmental Health Sciences R, Medicine, Board on Population $\mathrm{H}$, Public Health P, Institute of M. The National Academies Collection: Reports funded by National Institutes of Health. Identifying and Reducing Environmental Health Risks of Chemicals in Our Society: Workshop Summary. Washington (DC): National Academies Press (US); 2014.

91. U.s. Environmental Protection Agency. Toxic Substances Control Act (TSCA) Substance Inventory. 10/19/19. Available at: https://www.epa.gov/tsca-inventory. Accessed 11/27/19, 2019.

92. United States Environmental Protection Agency U. Toxic Release Inventory Program. Available at: https://www.epa.gov/toxics-release-inventory-tri-program, 2018.

93. Wilson SM, Fraser-Rahim $\mathrm{H}$, Williams $\mathrm{E}$, et al. Assessment of the distribution of toxic release inventory facilities in metropolitan Charleston: an environmental justice case study. Am J Public Health. Oct 2012;102(10):1974-1980.

94. Johnson R, Ramsey-White K, Fuller $\mathrm{CH}$. Socio-demographic Differences in Toxic Release Inventory Siting and Emissions in Metro Atlanta. Int J Environ Res Public Health. Jul $232016 ; 13(8)$.

95. Neumann CM, Forman DL, Rothlein JE. Hazard screening of chemical releases and environmental equity analysis of populations proximate to toxic release inventory facilities in Oregon. Environ Health Perspect. Apr 1998;106(4):217-226.

96. Aoyagi $\mathrm{H}$, Ogunseitan OA. Toxic releases and risk disparity: a spatiotemporal model of industrial ecology and social empowerment. Int J Environ Res Public Health. Jun 2 2015;12(6):6300-6318.

97. Taylor S, Edwards SJ, Walker TR. A toxicity-based analysis of Canada's National Pollutant Release Inventory (NPRI): a case study in Nova Scotia. Environ Sci Pollut Res Int. Jan 2020;27(2):2238-2247.

98. Lim SR, Lam CW, Schoenung JM. Priority screening of toxic chemicals and industry sectors in the U.S. toxics release inventory: a comparison of the life cycle impact-based and risk-based assessment tools developed by U.S. EPA. J Environ Manage. Sep 2011;92(9):2235-2240.

99. Lim SR, Lam CW, Schoenung JM. Quantity-based and toxicity-based evaluation of the U.S. Toxics Release Inventory. J Hazard Mater. Jun 15 2010;178(1-3):49-56.

100. Bulka C, Nastoupil LJ, Koff JL, et al. Relations Between Residential Proximity to EPADesignated Toxic Release Sites and Diffuse Large B-Cell Lymphoma Incidence. South Med J. Oct 2016;109(10):606-614.

101. Boeglin ML, Wessels $D$, Henshel D. An investigation of the relationship between air emissions of volatile organic compounds and the incidence of cancer in Indiana counties. Environmental Research. 2006/02/01/ 2006;100(2):242-254. 
102. Hanchette $\mathrm{C}$, Zhang $\mathrm{CH}$, Schwartz GG. Ovarian Cancer Incidence in the U.S. and Toxic Emissions from Pulp and Paper Plants: A Geospatial Analysis. Int J Environ Res Public Health. Jul 31 2018;15(8).

103. Choi HS, Shim YK, Kaye WE, Ryan PB. Potential residential exposure to toxics release inventory chemicals during pregnancy and childhood brain cancer. Environ Health Perspect. Jul 2006;114(7):1113-1118.

104. Hendryx M, Fedorko $\mathrm{E}$. The relationship between toxics release inventory discharges and mortality rates in rural and urban areas of the United States. $J$ Rural Health. Winter 2011;27(4):358-366.

105. Chen BC, Luo J, Hendryx M. Zinc compound air releases from Toxics Release Inventory facilities and cardiovascular disease mortality rates. Environ Res. Oct 2015;142:96-103.

106. United States Department of Health and Human Services (US DHHS), Centers for Disease Control and Prevention (CDC), National Center for Health Statistics (NCHS). Underlying Cause of Death 1999-2018 on CDC WONDER Online Database; 2020.

107. Research TNBoE. Census U.S. Intercensal County Population Data by Age, Sex, Race, and Hispanic Origin. Jean Roth. 10/17/2016. Available at:

https://data.nber.org/data/census-intercensal-county-population-age-sex-racehispanic.html, 2020.

108. Bureau USC. Population and Housing Unit Estimates Datasets. In: Bureau USC, ed: U.S. Census Bureau; 2000.

109. U.S. Census Bureau. Population and Housing Unit Estimates Datasets; 2010.

110. University of Washington. Institute for Health Metrics and Evaluation (IHME),. University of Washington. Available at: healthdata.org, 2020.

111. Dwyer-Lindgren L, Mokdad AH, Srebotnjak T, Flaxman AD, Hansen GM, Murray CJL. Cigarette smoking prevalence in US counties: 1996-2012. Population Health Metrics. 2014/03/24 2014;12(1):5.

112. Dwyer-Lindgren L, Flaxman AD, Ng M, Hansen GM, Murray CJL, Mokdad AH. Drinking Patterns in US Counties From 2002 to 2012. American journal of public health. 2015;105(6):1120-1127.

113. North America Land Data Assimilation System (NLDAS). Daily Air Temperatures and Heat Index, years 1979-2011. CDC Wonder; 2012.

114. Kim S-Y, Bechle M, Hankey S, Sheppard L, Szpiro AA, Marshall JD. Concentrations of criteria pollutants in the contiguous U.S., 1979 - 2015: Role of prediction model parsimony in integrated empirical geographic regression. PLOS ONE. 2020;15(2):e0228535.

115. U.S. Environmental Protection Agency NCUSRP, Center for International Earth Science Information Network,. U.S. EPA National Priorities List (NPL) Sites Point Data with CIESIN Modifications. In: (SEDAC) NSDaAC, ed. 2 ed. Palisades, NY; 2014.

116. Ingram DD Franco SJ. NCHS Urban-Rural Classification Scheme for Counties: National Center for Health Statistics; 2012.

117. Littell RC. SAS for mixed models. 2nd ed. ed. Cary, N.C.: SAS Institute; 2006.

118. Patetta M. Longitudinal Data Analysis with Discrete and Continous Responses Course Notes. Cary, NC: SAS Institute Inc.; 2014.

119. Wickham et al. Welcome to the Tidyverse. Journal of Open Source Software. 2019;4(43).

120. R Core Team. R: A language and environment for statistical computing. R Foundation for Statistical Computing, Vienna, Austria. 2019.

121. Fujii $\mathrm{H}$, Managi $\mathrm{S}$, Kawahara $\mathrm{H}$. The pollution release and transfer register system in the U.S. and Japan: an analysis of productivity. Journal of Cleaner Production. 2011/08/01/ 2011;19(12):1330-1338.

122. Environmental Protection Agency. TRI National Analysis Materials 2012.

123. Xu L, Polya DA, Li Q, Mondal D. Association of low-level inorganic arsenic exposure from rice with age-standardized mortality risk of cardiovascular disease (CVD) in England and Wales. Sci Total Environ. Nov 15 2020;743:140534.

124. Moon KA, Oberoi S, Barchowsky A, et al. A dose-response meta-analysis of chronic arsenic exposure and incident cardiovascular disease. Int J Epidemiol. Dec 1 2017;46(6):1924-1939. 
125. Marsh GM, Esmen NA, Buchanich JM, Youk AO. Mortality patterns among workers exposed to arsenic, cadmium, and other substances in a copper smelter. Am J Ind Med. Aug 2009;52(8):633-644.

126. Larsson SC, Wolk A. Urinary cadmium and mortality from all causes, cancer and cardiovascular disease in the general population: systematic review and meta-analysis of cohort studies. Int J Epidemiol. Jun 2016;45(3):782-791.

127. Humblet O, Birnbaum L, Rimm E, Mittleman MA, Hauser R. Dioxins and cardiovascular disease mortality. Environ Health Perspect. Nov 2008;116(11):1443-1448.

128. Brown L, Lynch M, Belova A, Klein R, Chiger A. Developing a Health Impact Model for Adult Lead Exposure and Cardiovascular Disease Mortality. Environ Health Perspect. Sep 2020;128(9):97005.

129. Zago AM, Faria NMX, Fávero JL, Meucci RD, Woskie S, Fassa AG. Pesticide exposure and risk of cardiovascular disease: A systematic review. Glob Public Health. Aug 20 2020:1-23.

130. Grandjean $\mathrm{P}$, Landrigan PJ. Developmental neurotoxicity of industrial chemicals. Lancet. Dec 16 2006;368(9553):2167-2178.

131. Grandjean P, Landrigan PJ. Neurobehavioural effects of developmental toxicity. The lancet neurology. 2014;13(3):330-338.

132. Landrigan PJ, Goldman LR. Children's vulnerability to toxic chemicals: a challenge and opportunity to strengthen health and environmental policy. Health Affairs. 2011;30(5):842-850.

133. Armor JN. Volatile Organic Compounds: An Overview. Environmental Catalysis. Vol 552: American Chemical Society; 1994:298-300.

134. World Health Organization W. Air Quality Guidelines for Europe. Copenhagen 2000.

135. Mizukoshi A, Kumagai K, Yamamoto N, et al. A Novel Methodology to Evaluate Health Impacts Caused by VOC Exposures Using Real-Time VOC and Holter Monitors. International Journal of Environmental Research and Public Health. 2010;7(12).

136. Wang BL, Takigawa T, Takeuchi A, et al. Unmetabolized VOCs in urine as biomarkers of low level exposure in indoor environments. J Occup Health. Mar 2007;49(2):104-110.

137. Shim YH, Ock JW, Kim Y-J, Kim Y, Kim SY, Kang D. Association between Heavy Metals, Bisphenol A, Volatile Organic Compounds and Phthalates and Metabolic Syndrome. International Journal of Environmental Research and Public Health. 2019;16(4):671.

138. Jain RB. Distributions of selected urinary metabolites of volatile organic compounds by age, gender, race/ethnicity, and smoking status in a representative sample of U.S. adults. Environmental Toxicology and Pharmacology. 9// 2015;40(2):471-479.

139. Centers for Disease Control and Prevention C. Laboratory Procedure Manual: Volatile Organic Compound (VOCs) Metabolites. In: Health E, ed. Atlanta, GA; 2011.

140. National Research Council. Human Biomonitoring for Environmental Chemicals. Washington D.C.: National Academy of Sceinces; 2006.

141. McGraw KE, Riggs DW, Rai S, et al. Exposure to Volatile Organic Compounds Acrolein, 1,3-Butadiene, and Crotonaldehyde - is Associated with Vascular Dysfunction. Environmental Research. 2021/02/24/ 2021:110903.

142. Axtell AL, Gomari FA, Cooke JP. Assessing endothelial vasodilator function with the Endo-PAT 2000. Journal of visualized experiments : JoVE. 2010(44):2167.

143. Xie Z, Lorkiewicz P, Riggs DW, Bhatnagar A, Srivastava S. Comprehensive, robust, and sensitive UPLC-MS/MS analysis of free biogenic monoamines and their metabolites in urine. Journal of Chromatography B. 2018/11/01/2018;1099:83-91.

144. Boyle EB, Viet SM, Wright DJ, et al. Assessment of Exposure to VOCs among Pregnant Women in the National Children's Study. Int J Environ Res Public Health. Mar 29 2016;13(4):376.

145. Lorkiewicz P, Riggs DW, Keith RJ, et al. Comparison of Urinary Biomarkers of Exposure in Humans Using Electronic Cigarettes, Combustible Cigarettes, and Smokeless Tobacco. Nicotine Tob Res. Jun 22018.

146. National Institute of Health (NIH). Assessing Cardiovascular Risk: National Heart, Lung, and Blood Institution; 2013. 
147. Keith RJ, Fetterman JL, Orimoloye OA, et al. Characterization of Volatile Organic Compound Metabolites in Cigarette Smokers, Electronic Nicotine Device Users, Dual Users, and Nonusers of Tobacco. Nicotine Tob Res. Feb 6 2020;22(2):264-272.

148. Avila-Tang E, Al-Delaimy WK, Ashley DL, et al. Assessing secondhand smoke using biological markers. Tob Control. May 2013;22(3):164-171.

149. Hayes AF. Introduction to mediation, moderation, and conditional process analysis: $A$ regression-based approach: Guilford publications; 2017.

150. Goldberg M, Silbergeld E. On multiple comparisons and on the design and interpretation of epidemiological studies of many associations. Environmental Research. 2011/11/01/ 2011;111(8):1007-1009.

151. Rothman KJ. No adjustments are needed for multiple comparisons. Epidemiology. Jan 1990;1(1):43-46.

152. Singh $M$, Kapoor A, Bhatnagar A. Oxidative and reductive metabolism of lipidperoxidation derived carbonyls. Chem Biol Interact. Jun 5 2015;234:261-273.

153. U.S. Environmental Protection Agency. National Air Toxics Assessment. Available at: http://www.epa.gov/ttn/atw/natamain/.

154. Perez CM, Hazari MS, Ledbetter AD, et al. Acrolein inhalation alters arterial blood gases and triggers carotid body-mediated cardiovascular responses in hypertensive rats. Inhal Toxicol. Jan 2015;27(1):54-63.

155. Perez CM, Ledbetter AD, Hazari MS, et al. Hypoxia stress test reveals exaggerated cardiovascular effects in hypertensive rats after exposure to the air pollutant acrolein. Toxicol Sci. Apr 2013;132(2):467-477.

156. Ismahil MA, Hamid $T$, Haberzettl $P$, et al. Chronic oral exposure to the aldehyde pollutant acrolein induces dilated cardiomyopathy. Am J Physiol Heart Circ Physiol. Nov $2011 ; 301(5): \mathrm{H} 2050-2060$.

157. O'Toole TE, Abplanalp W, Li X, et al. Acrolein decreases endothelial cell migration and insulin sensitivity through induction of let-7a. Toxicol Sci. Aug 1 2014;140(2):271-282.

158. Wheat LA, Haberzettl $P$, Hellmann J, et al. Acrolein inhalation prevents vascular endothelial growth factor-induced mobilization of Flk-1+/Sca-1+ cells in mice. Arterioscler Thromb Vasc Biol. Jul 2011;31(7):1598-1606.

159. Awe SO, Adeagbo ASO, D'Souza SE, Bhatnagar A, Conklin DJ. Acrolein induces vasodilatation of rodent mesenteric bed via an EDHF-dependent mechanism. Toxicology and Applied Pharmacology. 2006/12/15/ 2006;217(3):266-276.

160. George J, Hussain M, Vadiveloo T, et al. Cardiovascular Effects of Switching From Tobacco Cigarettes to Electronic Cigarettes. Journal of the American College of Cardiology. 2019;74(25):3112-3120.

161. Miyata S, Noda A, Ito Y, lizuka R, Shimokata K. Smoking acutely impaired endothelial function in healthy college students. Acta Cardiologica. 2015/06/01 2015;70(3):282-285.

162. Esen AM, Barutcu I, Acar M, et al. Effect of Smoking on Endothelial Function and Wall Thickness of Brachial Artery. Circulation Journal. 2004;68(12):1123-1126.

163. Carnevale R, Sciarretta S, Violi F, et al. Acute Impact of Tobacco vs Electronic Cigarette Smoking on Oxidative Stress and Vascular Function. Chest. 2016/09/01/ 2016;150(3):606-612.

164. Fetterman Jessica L, Keith Rachel J, Palmisano Joseph N, et al. Alterations in Vascular Function Associated With the Use of Combustible and Electronic Cigarettes. Journal of the American Heart Association. 2020/05/05 2020;9(9):e014570.

165. Fetterman JL, Weisbrod RM, Feng B, et al. Flavorings in Tobacco Products Induce Endothelial Cell Dysfunction. Arteriosclerosis, thrombosis, and vascular biology. 2018;38(7):1607-1615.

166. Kuntic M, Oelze M, Steven S, et al. Short-term e-cigarette vapour exposure causes vascular oxidative stress and dysfunction: evidence for a close connection to brain damage and a key role of the phagocytic NADPH oxidase (NOX-2). European heart journal. 2020;41(26):2472-2483.

167. Snow SJ, McGee MA, Henriquez A, et al. Respiratory Effects and Systemic Stress Response Following Acute Acrolein Inhalation in Rats. Toxicological sciences : an official journal of the Society of Toxicology. 2017;158(2):454-464. 
168. Divine BJ, Hartman CM. Mortality update of butadiene production workers. Toxicology. 1996/10/28/ 1996;113(1):169-181.

169. Penn A, Snyder CA. 1,3-Butadiene exposure and cardiovascular disease. Mutation Research/Fundamental and Molecular Mechanisms of Mutagenesis. 2007/08/01/ 2007;621(1):42-49.

170. Matanoski GM, Santos-Burgoa C, Schwartz L. Mortality of a cohort of workers in the styrene-butadiene polymer manufacturing industry (1943-1982). Environ Health Perspect. Jun 1990;86:107-117.

171. National Center for Biotechnology Information. 1,3-Butadiene. PubChem Database. Available at: https://pubchem.ncbi.nlm.nih.gov/compound/1 3-Butadiene, 24 July 2020.

172. Shin HH, Jones $\mathrm{P}$, Brook R, Bard R, Oliver K, Williams R. Associations between personal exposures to VOCs and alterations in cardiovascular physiology: Detroit Exposure and Aerosol Research Study (DEARS). Atmospheric Environment. 2015/03/01/ 2015;104(Supplement C):246-255.

173. Soeteman-Hernández LG, Bos PMJ, Talhout R. Tobacco smoke-related health effects induced by 1,3-butadiene and strategies for risk reduction. Toxicological sciences : an official journal of the Society of Toxicology. 2013;136(2):566-580.

174. Bhatnagar A. Cardiovascular pathophysiology of environmental pollutants. Am J Physiol Heart Circ Physiol. Feb 2004;286(2):H479-485.

175. Conklin DJ. Acute cardiopulmonary toxicity of inhaled aldehydes: role of TRPA1. Ann NY Acad Sci. Jun 2016;1374(1):59-67.

176. O'Toole TE, Zheng YT, Hellmann J, Conklin DJ, Barski O, Bhatnagar A. Acrolein activates matrix metalloproteinases by increasing reactive oxygen species in macrophages. Toxicol Appl Pharmacol. Apr 15 2009;236(2):194-201.

177. Conklin DJ, Ogunwale MA, Chen Y, et al. Electronic cigarette-generated aldehydes: The contribution of e-liquid components to their formation and the use of urinary aldehyde metabolites as biomarkers of exposure. Aerosol science and technology : the journal of the American Association for Aerosol Research. 2018;52(11):1219-1232.

178. Grant RL, Jenkins AF. Use of In Vivo and In Vitro Data to Derive a Chronic Reference Value for Crotonaldehyde Based on Relative Potency to Acrolein. Journal of toxicology and environmental health. Part B, Critical reviews. 2015;18(7-8):327-343.

179. National Center for Biotechnology Information. Crotonaldehyde. PubChem Database. Available at: https://pubchem.ncbi.nlm.nih.gov/compound/Crotonaldehyde Accessed 25 July 2020.

180. Lynch J, Jin L, Richardson A, et al. Acute and chronic vascular effects of inhaled crotonaldehyde in mice: Role of TRPA1. Toxicology and applied pharmacology. 2020/09// 2020;402:115120.

181. Koppaka V, Thompson DC, Chen Y, et al. Aldehyde dehydrogenase inhibitors: a comprehensive review of the pharmacology, mechanism of action, substrate specificity, and clinical application. Pharmacological reviews. 2012;64(3):520-539.

182. LoPachin RM, Barber DS, Gavin T. Molecular mechanisms of the conjugated alpha,betaunsaturated carbonyl derivatives: relevance to neurotoxicity and neurodegenerative diseases. Toxicological sciences : an official journal of the Society of Toxicology. 2008;104(2):235-249.

183. Rees JN, Florang VR, Anderson DG, Doorn JA. Lipid peroxidation products inhibit dopamine catabolism yielding aberrant levels of a reactive intermediate. Chem Res Toxicol. Oct 2007;20(10):1536-1542.

184. Frieden TR. CDC health disparities and inequalities report-United States, 2013. Foreword. MMWR supplements. 2013;62(3):1-2.

185. Henning RJ, Johnson GT, Coyle JP, Harbison RD. Acrolein Can Cause Cardiovascular Disease: A Review. Cardiovascular Toxicology. 2017/07/01 2017;17(3):227-236.

186. Alwis KU, deCastro BR, Morrow JC, Blount BC. Acrolein Exposure in U.S. Tobacco Smokers and Non-Tobacco Users: NHANES 2005-2006. Environmental health perspectives. 2015;123(12):1302-1308.

187. Mills PJ, Dimsdale JE, Ziegler MG, Nelesen RA. Racial differences in epinephrine and beta 2-adrenergic receptors. Hypertension. Jan 1995;25(1):88-91. 
188. Sherwood A, Hill LK, Blumenthal JA, Johnson KS, Hinderliter AL. Race and sex differences in cardiovascular $\alpha$-adrenergic and $\beta$-adrenergic receptor responsiveness in men and women with high blood pressure. Journal of hypertension. 2017;35(5):975-981.

189. Bhatnagar A. Environmental cardiology: studying mechanistic links between pollution and heart disease. Circ Res. Sep 29 2006;99(7):692-705.

190. Riggs DW, Yeager RA, Bhatnagar A. Defining the Human Envirome: An Omics Approach for Assessing the Environmental Risk of Cardiovascular Disease. Circ Res. Apr 27 2018;122(9):1259-1275.

191. Forouzanfar MH, Alexander L, Anderson HR, et al. Global, regional, and national comparative risk assessment of 79 behavioural, environmental and occupational, and metabolic risks or clusters of risks in 188 countries, 1990\&\#x2013;2013: a systematic analysis for the Global Burden of Disease Study 2013. The Lancet.

2015;386(10010):2287-2323.

192. Navas-Acien A, Selvin E, Sharrett AR, Calderon-Aranda E, Silbergeld E, Guallar E. Lead, cadmium, smoking, and increased risk of peripheral arterial disease. Circulation. Jun 29 2004;109(25):3196-3201.

193. Navas-Acien A, Silbergeld EK, Sharrett R, Calderon-Aranda E, Selvin E, Guallar E. Metals in urine and peripheral arterial disease. Environ Health Perspect. Feb 2005;113(2):164-169.

194. Kaufman JD, Adar SD, Allen RW, et al. Prospective study of particulate air pollution exposures, subclinical atherosclerosis, and clinical cardiovascular disease: The MultiEthnic Study of Atherosclerosis and Air Pollution (MESA Air). Am J Epidemiol. Nov 1 2012;176(9):825-837.

195. Kaufman JD, Adar SD, Barr RG, et al. Association between air pollution and coronary artery calcification within six metropolitan areas in the USA (the Multi-Ethnic Study of Atherosclerosis and Air Pollution): a longitudinal cohort study. Lancet. Aug 13 2016;388(10045):696-704.

196. National Institute of Environmental Health Science (NIEHS). Strategic Plan 2018-2023. 2/28/2019. Available at: https://www.niehs.nih.gov/about/strategicplan/index.cfm. Accessed 11/30/2019, 2019.

197. A. Gibson E, Nunez Y, Abuawad A, et al. An overview of methods to address distinct research questions on environmental mixtures: an application to persistent organic pollutants and leukocyte telomere length. Environmental Health: A Global Access Science Source. 2019;18(1):N.PAG-N.PAG.

198. Hoerl AE, Kennard RW. Ridge Regression: Biased Estimation for Nonorthogonal Problems. Technometrics. 1970/02/01 1970;12(1):55-67.

199. Tibshirani R. Regression shrinkage and selection via the lasso. Journal of the Royal Statistical Society: Series B (Methodological). 1996;58(1):267-288.

200. Zou H, Hastie T. Regularization and Variable Selection via the Elastic Net. Journal of the Royal Statistical Society. Series B (Statistical Methodology). 2005;67(2):301-320.

201. Hastie T, Qian, J. GImnet Vignette. Standford University. Available at: https://web.stanford.edu/ hastie/glmnet/glmnet alpha.html.

202. Gibson EA, Goldsmith J, Kioumourtzoglou MA. Complex Mixtures, Complex Analyses: an Emphasis on Interpretable Results. Curr Environ Health Rep. Jun 2019;6(2):53-61.

203. Bobb JF, Valeri L, Claus Henn B, et al. Bayesian kernel machine regression for estimating the health effects of multi-pollutant mixtures. Biostatistics. Jul 2015;16(3):493508.

204. Friedman J HT, Tibshirani R. Regularization Paths for Generalized Linear Models via Coordinate Descent. Journal of Statistical Software. 2010;33(1):1-22.

205. Bobb JF. Bayesian Kernel Machine Regression [R package bkmr

version 0.2.0]. Comprehensive R Archive Network (CRAN). Available at: https://cran.rproject.org/web/packages/bkmr/index.html. Accessed 18 July 2020.

206. Grazuleviciene R, Kapustinskiene V, Vencloviene J, Buinauskiene J, Nieuwenhuijsen MJ. Risk of congenital anomalies in relation to the uptake of trihalomethane from drinking water during pregnancy. Occupational and Environmental Medicine. 2013;70(4):274-282. 
207. Wright JM, Evans A, Kaufman JA, Rivera-Núñez Z, Narotsky MG. Disinfection ByProduct Exposures and the Risk of Specific Cardiac Birth Defects. Environ Health Perspect. Feb 2017;125(2):269-277.

208. Colman J, Rice GE, Wright JM, et al. Identification of developmentally toxic drinking water disinfection byproducts and evaluation of data relevant to mode of action. Toxicol Appl Pharmacol. Jul 15 2011;254(2):100-126.

209. Nieuwenhuijsen MJ, Martinez D, Grellier J, et al. Chlorination disinfection by-products in drinking water and congenital anomalies: review and meta-analyses. Environmental health perspectives. 2009;117(10):1486-1493.

210. Kaur N, Starling AP, Calafat AM, et al. Longitudinal association of biomarkers of pesticide exposure with cardiovascular disease risk factors in youth with diabetes. Environmental Research. 2020/02/01/2020;181:108916.

211. Zitko V. Toxicity and pollution potential of thallium. Science of The Total Environment. 1975/07/01/ 1975;4(2):185-192.

212. Carroll RJ, Ruppert, D., Stefanski, L.A., \& Crainiceanu, C.M. Measurement Error in Nonlinear Models: A Modern Perspective: Chapman and Hall/CRC; 2006.

213. Pollack AZ, Perkins NJ, Mumford SL, Ye A, Schisterman EF. Correlated Biomarker Measurement Error: An Important Threat to Inference in Environmental Epidemiology. American Journal of Epidemiology. 2013;177(1):84-92.

214. U.S. Environmental Protection Agency. The Benefits and Costs of the Clean Air Act from 1990 to 20202011.

215. Health Effects Institute. Development of Statistical Methods for Multipollutant Research. Boston, MA, 2015.

216. Jain RB. Selected volatile organic compounds as biomarkers for exposure to tobacco smoke. Biomarkers. 2016;21(4):342-346. 


\section{APPENDICES}

APPENDIX A

\begin{tabular}{|c|c|c|c|c|}
\hline Variable & Mean & Std Dev & Min & Max \\
\hline _1_3_CHLOROALLYL_3_5_7_TRIAZA_ & 46.7 & 1940.3 & 0 & 92183 \\
\hline _1_CHLORO_1_1_DIFLUOROETHANE & 66.1 & 2552.5 & 0 & 121220 \\
\hline _1_CHLORO_1_1_2_2_TETRAFLUOROETH & 3.6 & 128.6 & 0 & 5938 \\
\hline _1_1_DICHLORO_1_FLUOROETHANE & 18.3 & 516.6 & 0 & 21908 \\
\hline _1_1_DIMETHYL_HYDRAZINE & 0.0 & 0.4 & 0 & 17.32 \\
\hline _1_1_1_TRICHLOROETHANE & 47.5 & 1008.1 & 0 & 36502 \\
\hline _1_1_1_2_TETRACHLORO_2_FLUOROETH & 0.0 & 0.2 & 0 & 10 \\
\hline _1_1_1_2_TETRACHLOROETHANE & 2.5 & 85.8 & 0 & 3430 \\
\hline _1_1_2_TRICHLOROETHANE & 9.9 & 219.0 & 0 & 8334 \\
\hline _1_1_2_2_TETRACHLOROETHANE & 1.9 & 44.6 & 0 & 1387.81 \\
\hline _1_2_BUTYLENE_OXIDE & 2.1 & 44.3 & 0 & 1376 \\
\hline _1_2_DIBROMO_3_CHLOROPROPANE & 0.0 & 0.2 & 0 & 10.78 \\
\hline _1_2_DIBROMOETHANE & 0.8 & 33.9 & 0 & 1619 \\
\hline _1_2_DICHLORO_1_1_DIFLUOROETHANE & 12.9 & 616.7 & 0 & 29580 \\
\hline _1_2_DICHLORO_1_1_2_TRIFLUOROETH & 46.1 & 2133.8 & 0 & 102300 \\
\hline _1_2_DICHLOROBENZENE & 27.6 & 1120.3 & 0 & 52950 \\
\hline _1_2_DICHLOROETHANE & 230.9 & 3835.8 & 0 & 115996.4 \\
\hline _1_2_DICHLOROETHYLENE & 3.7 & 120.3 & 0 & 5507 \\
\hline _1_2_DICHLOROPROPANE & 30.4 & 1162.3 & 0 & 55197 \\
\hline _1_2_DIPHENYLHYDRAZINE & 0.0 & 0.4 & 0 & 15.06 \\
\hline _1_2_PHENYLENEDIAMINE & 1.6 & 66.6 & 0 & 3168 \\
\hline _1_2_3_TRICHLOROPROPANE & 2.7 & 96.3 & 0 & 4173 \\
\hline _1_2_4_TRICHLOROBENZENE & 3.8 & 118.9 & 0 & 5450 \\
\hline _1_2_4_TRIMETHYLBENZENE & 2690.5 & 19369.0 & 0 & 408713 \\
\hline _1_3_BUTADIENE & 547.2 & 10591.1 & 0 & 345413.8 \\
\hline _1_3_DICHLORO_1_1_2_2_3_PENTAFLU & 0.0 & 1.5 & 0 & 73 \\
\hline _1_3_DICHLOROBENZENE & 0.0 & 0.6 & 0 & 22.49 \\
\hline _1_3_DICHLOROPROPYLENE & 3.2 & 57.4 & 0 & 1845 \\
\hline _1_3_PHENYLENEDIAMINE & 14.4 & 424.9 & 0 & 17403.8 \\
\hline _1_4_DICHLORO_2_BUTENE & 2.6 & 121.4 & 0 & 5824 \\
\hline _1_4_DICHLOROBENZENE & 18.1 & 618.2 & 0 & 27128 \\
\hline _1_4_DIOXANE & 513.4 & 21612.4 & 0 & 1035580 \\
\hline _2_ACETYLAMINOFLUORENE & 0.1 & 3.3 & 0 & 158.44 \\
\hline _2_CHLORO_1_1_1_TRIFLUOROETHANE & 19.5 & 606.7 & 0 & 25028 \\
\hline _2_CHLORO_1_1_1_2_TETRAFLUOROETH & 166.5 & 3959.2 & 0 & 129000 \\
\hline _2_ETHOXYETHANOL & 16.7 & 587.3 & 0 & 27406 \\
\hline _2_MERCAPTOBENZOTHIAZOLE & 16.8 & 427.6 & 0 & 18788 \\
\hline 2_METHOXYETHANOL & 38.3 & 1674.5 & 0 & 80237.87 \\
\hline _2_METHYLLACTONITRILE & 104.1 & 4882.1 & 0 & 234144 \\
\hline _2_METHYLPYRIDINE & 25.4 & 786.4 & 0 & 27783 \\
\hline
\end{tabular}




\begin{tabular}{|c|c|c|c|c|}
\hline _2_NITROPHENOL & 1.5 & 69.7 & 0 & 3344 \\
\hline _2_NITROPROPANE & 14.4 & 684.1 & 0 & 32814 \\
\hline _2_PHENYLPHENOL & 1.5 & 64.2 & 0 & 3071 \\
\hline _2_2_BIS_BROMOMETHYL_1_3_PROPAN & 0.1 & 3.0 & 0 & 145 \\
\hline _2_2_DICHLORO_1_1_1_TRIFLUOROETH & 91.3 & 1755.5 & 0 & 53928 \\
\hline _2_3_DICHLOROPROPENE & 2.0 & 88.9 & 0 & 4255 \\
\hline 2_4_D & 173.6 & 5954.4 & 0 & 277319 \\
\hline _2_4_D_2_ETHYLHEXYL_ESTER & 0.5 & 15.6 & 0 & 612 \\
\hline _2_4_D_BUTOXYETHYL_ESTER & 0.6 & 28.8 & 0 & 1380 \\
\hline _2_4_D_SODIUM_SALT & 0.0 & 0.2 & 0 & 9 \\
\hline _2_4_DIAMINOTOLUENE & 1.1 & 38.1 & 0 & 1710.7 \\
\hline _2_4_DICHLOROPHENOL & 124.7 & 5417.4 & 0 & 259321 \\
\hline _2_4_DIMETHYLPHENOL & 21.4 & 845.9 & 0 & 40470.4 \\
\hline _2_4_DINITROPHENOL & 3.4 & 121.8 & 0 & 5500 \\
\hline _2_4_DINITROTOLUENE & 3.1 & 137.5 & 0 & 6576 \\
\hline _2_4_DITHIOBIURET & 0.0 & 0.2 & 0 & 7.49 \\
\hline _2_4_5_TRICHLOROPHENOL & 0.0 & 1.0 & 0 & 41.97 \\
\hline _2_4_6_TRICHLOROPHENOL & 0.0 & 0.8 & 0 & 29 \\
\hline _2_6_DINITROTOLUENE & 0.8 & 39.9 & 0 & 1915 \\
\hline _2_6_XYLIDINE & 0.0 & 1.7 & 0 & 80 \\
\hline _3_CHLORO_1_1_1_TRIFLUOROPROPANE & 0.1 & 3.5 & 0 & 169 \\
\hline _3_CHLORO_2_METHYL_1_PROPENE & 0.0 & 1.4 & 0 & 52 \\
\hline _3_CHLOROPROPIONITRILE & 0.0 & 0.2 & 0 & 8 \\
\hline _3_IODO_2_PROPYNYL_BUTYLCARBAMAT & 106.9 & 4939.5 & 0 & 236887 \\
\hline _3_3_DICHLOROBENZIDINE & 0.0 & 0.3 & 0 & 13.52 \\
\hline _3_3_DICHLOROBENZIDINE_DIHYDROC & 0.0 & 0.0 & 0 & 1.4 \\
\hline _3_3_DIMETHOXYBENZIDINE & 0.0 & 0.3 & 0 & 13.62 \\
\hline _3_3_DIMETHOXYBENZIDINE_DIHYDRO & 0.1 & 5.3 & 0 & 255 \\
\hline 3_3_DIMETHYLBENZIDINE & 0.0 & 0.3 & 0 & 13.6 \\
\hline _3_3_DICHLORO_1_1_1_2_2_PENTAFLU & 1.6 & 77.2 & 0 & 3704 \\
\hline _4_AMINOAZOBENZENE & 0.4 & 17.0 & 0 & 814 \\
\hline _4_AMINOBIPHENYL & 0.0 & 0.2 & 0 & 11 \\
\hline _4_DIMETHYLAMINOAZOBENZENE & 0.0 & 0.3 & 0 & 13.52 \\
\hline _4_NITROPHENOL & 0.2 & 7.7 & 0 & 371 \\
\hline _4_4_DIAMINODIPHENYL_ETHER & 0.4 & 15.7 & 0 & 752.6 \\
\hline _4_4_ISOPROPYLIDENEDIPHENOL & 715.2 & 12516.3 & 0 & 405328 \\
\hline _4_4_METHYLENEBIS_2_CHLOROANILI & 0.2 & 7.0 & 0 & 250 \\
\hline _4_4_METHYLENEDIANILINE & 55.4 & 2528.7 & 0 & 121267 \\
\hline _4_6_DINITRO_O_CRESOL & 0.0 & 0.0 & 0 & 1 \\
\hline _5_NITRO_O_TOLUIDINE & 0.0 & 0.4 & 0 & 21.06 \\
\hline ABAMECTIN & 6.1 & 265.9 & 0 & 12684.4 \\
\hline ACEPHATE & 1.9 & 90.9 & 0 & 4359 \\
\hline ACETALDEHYDE & 4125.8 & 18828.5 & 0 & 316488 \\
\hline ACETAMIDE & 574.7 & 20107.5 & 0 & 938500 \\
\hline
\end{tabular}




\begin{tabular}{|c|c|c|c|c|}
\hline ACETONITRILE & 7619.5 & 189245.6 & 0 & 6601129 \\
\hline ACETOPHENONE & 277.4 & 8960.5 & 0 & 397371.1 \\
\hline ACIFLUORFEN_SODIUM_SALT & 0.0 & 0.3 & 0 & 12.3 \\
\hline ACROLEIN & 358.6 & 11549.1 & 0 & 551074 \\
\hline ACRYLAMIDE & 2255.5 & 57407.3 & 0 & 2111600 \\
\hline ACRYLIC_ACID & 1642.4 & 60080.8 & 0 & 2855236 \\
\hline ACRYLONITRILE & 4736.9 & 170216.6 & 0 & 7801250 \\
\hline ALACHLOR & 0.0 & 0.6 & 0 & 28 \\
\hline ALDICARB & 0.0 & 1.5 & 0 & 69.9 \\
\hline ALDRIN & 0.4 & 17.1 & 0 & 820 \\
\hline ALLYL_ALCOHOL & 210.8 & 5352.4 & 0 & 226675.9 \\
\hline ALLYL_CHLORIDE & 11.6 & 281.7 & 0 & 12328 \\
\hline ALLYLAMINE & 3.0 & 88.6 & 0 & 3472 \\
\hline ALPHA_NAPHTHYLAMINE & 0.0 & 0.3 & 0 & 13.59 \\
\hline ALUMINUM_FUME_OR_DUST_ & 6908.8 & 73851.6 & 0 & 1954989 \\
\hline ALUMINUM_OXIDE_FIBROUS_FORMS_ & 4691.3 & 88376.7 & 0 & 3032740 \\
\hline ALUMINUM_PHOSPHIDE & 0.0 & 2.1 & 0 & 99.55 \\
\hline AMETRYN & 44.9 & 1527.9 & 0 & 63412 \\
\hline AMITROLE & 0.0 & 0.3 & 0 & 15.15 \\
\hline AMMONIA & 73401.7 & 494953.2 & 0 & $\begin{array}{c}1177202 \\
3\end{array}$ \\
\hline ANILAZINE & 100.0 & 4795.1 & 0 & 230015 \\
\hline ANILINE & 752.4 & 31841.2 & 0 & 1524786 \\
\hline ANTHRACENE & 20.5 & 529.7 & 0 & 23727.3 \\
\hline ANTIMONY & 360.3 & 12367.6 & 0 & 584866.5 \\
\hline ANTIMONY_COMPOUNDS & 2459.5 & 32822.1 & 0 & 963955 \\
\hline ARSENIC & 587.2 & 11805.4 & 0 & 434362.4 \\
\hline ARSENIC_COMPOUNDS & 33190.1 & 1112963.0 & 0 & $\begin{array}{c}5100052 \\
6\end{array}$ \\
\hline ASBESTOS_FRIABLE_ & 6119.1 & 181833.6 & 0 & 8559260 \\
\hline ATRAZINE & 374.7 & 9920.6 & 0 & 412017 \\
\hline BARIUM & 2514.5 & 51997.9 & 0 & 2014596 \\
\hline BARIUM_COMPOUNDS & 88172.7 & 454228.4 & 0 & $\begin{array}{c}1253163 \\
3\end{array}$ \\
\hline BENFLURALIN & 0.5 & 21.5 & 0 & 1000 \\
\hline BENZAL_CHLORIDE & 0.1 & 3.9 & 0 & 186 \\
\hline BENZENE & 1778.2 & 13834.3 & 0 & 456093.5 \\
\hline BENZIDINE & 0.0 & 0.4 & 0 & 13.1 \\
\hline BENZO_G_H_I_PERYLENE & 52.8 & 1020.1 & 0 & 33490.75 \\
\hline BENZOIC_TRICHLORIDE & 0.0 & 0.3 & 0 & 13.93 \\
\hline BENZOYL_CHLORIDE & 1.3 & 40.1 & 0 & 1817 \\
\hline BENZOYL_PEROXIDE & 18.4 & 423.9 & 0 & 13804 \\
\hline BENZYL_CHLORIDE & 6.7 & 135.1 & 0 & 4473 \\
\hline BERYLLIUM & 0.7 & 19.8 & 0 & 790 \\
\hline BERYLLIUM_COMPOUNDS & 209.3 & 2434.4 & 0 & 61981.75 \\
\hline BETA_NAPHTHYLAMINE & 0.0 & 0.3 & 0 & 13.55 \\
\hline
\end{tabular}




\begin{tabular}{|c|c|c|c|c|}
\hline BIFENTHRIN & 1.3 & 63.3 & 0 & 3037 \\
\hline BIPHENYL & 197.9 & 3980.5 & 0 & 131408 \\
\hline BIS_2_CHLORO_1_METHYLETHYL_ETH & 0.0 & 1.8 & 0 & 86 \\
\hline BIS_2_CHLOROETHOXY_METHANE & 0.0 & 0.5 & 0 & 21.59 \\
\hline BIS_2_CHLOROETHYL_ETHER & 0.1 & 3.8 & 0 & 168.0524 \\
\hline BIS_CHLOROMETHYL_ETHER & 0.0 & 0.2 & 0 & 7.49 \\
\hline BORON_TRICHLORIDE & 0.4 & 14.3 & 0 & 657.2 \\
\hline BORON_TRIFLUORIDE & 5.1 & 159.4 & 0 & 5656 \\
\hline BROMINE & 193.2 & 6633.8 & 0 & 312000 \\
\hline BROMOCHLORODIFLUOROMETHANE & 1.4 & 47.9 & 0 & 2081 \\
\hline BROMOFORM & 4.1 & 195.8 & 0 & 9392 \\
\hline BROMOMETHANE & 120.8 & 2656.4 & 0 & 94400 \\
\hline BROMOTRIFLUOROMETHANE & 3.0 & 88.5 & 0 & 3238 \\
\hline BROMOXYNIL & 0.0 & 0.4 & 0 & 17.5 \\
\hline BROMOXYNIL_OCTANOATE & 0.0 & 1.1 & 0 & 54 \\
\hline BRUCINE & 0.0 & 0.2 & 0 & 7.49 \\
\hline BUTYL_ACRYLATE & 182.6 & 4650.6 & 0 & 156967.7 \\
\hline BUTYRALDEHYDE & 170.5 & 3586.2 & 0 & 141549 \\
\hline C_I_DIRECT_BLACK_38 & 0.0 & 0.3 & 0 & 11 \\
\hline C_I_DIRECT_BLUE_218 & 0.1 & 5.4 & 0 & 255 \\
\hline C_I_SOLVENT_YELLOW_34 & 0.0 & 0.3 & 0 & 13.82 \\
\hline CADMIUM & 181.3 & 3963.8 & 0 & 156611 \\
\hline CADMIUM_COMPOUNDS & 3984.0 & 139831.5 & 0 & 6640948 \\
\hline CALCIUM_CYANAMIDE & 0.0 & 0.4 & 0 & 17.63 \\
\hline CAPTAN & 0.0 & 0.9 & 0 & 27 \\
\hline CARBARYL & 0.5 & 16.8 & 0 & 743.92 \\
\hline CARBOFURAN & 0.1 & 1.9 & 0 & 89 \\
\hline CARBON_DISULFIDE & 4041.6 & 94352.4 & 0 & 3752500 \\
\hline CARBON_TETRACHLORIDE & 100.1 & 2173.2 & 0 & 72999.47 \\
\hline CARBONYL_SULFIDE & 5880.2 & 104835.2 & 0 & 4100000 \\
\hline CARBOXIN & 0.0 & 0.3 & 0 & 12.66 \\
\hline CATECHOL & 6.9 & 101.5 & 0 & 3782.4 \\
\hline CERTAIN_GLYCOL_ETHERS & 7029.2 & 37529.1 & 0 & 915213.1 \\
\hline CHLORDANE & 0.6 & 20.5 & 0 & 949.02 \\
\hline CHLORIMURON_ETHYL & 0.0 & 0.2 & 0 & 9.27 \\
\hline CHLORINE & 2567.6 & 86506.5 & 0 & 4138718 \\
\hline CHLORINE_DIOXIDE & 214.9 & 2586.2 & 0 & 73690 \\
\hline CHLOROACETIC_ACID & 473.0 & 22576.6 & 0 & 1082972 \\
\hline CHLOROBENZENE & 217.7 & 7281.2 & 0 & 337786 \\
\hline CHLOROBENZILATE & 0.0 & 0.3 & 0 & 13.73 \\
\hline CHLORODIFLUOROMETHANE & 1014.3 & 21768.6 & 0 & 850088.9 \\
\hline CHLOROETHANE & 69.4 & 1629.3 & 0 & 67186 \\
\hline CHLOROFORM & 197.2 & 2508.0 & 0 & 57486.68 \\
\hline CHLOROMETHANE & 558.6 & 6669.6 & 0 & 216252 \\
\hline
\end{tabular}




\begin{tabular}{|c|c|c|c|c|}
\hline CHLOROMETHYL_METHYL_ETHER & 0.5 & 25.0 & 0 & 1200 \\
\hline CHLOROPHENOLS & 30.2 & 1406.1 & 0 & 67439.92 \\
\hline CHLOROPICRIN & 2.0 & 49.7 & 0 & 2070.91 \\
\hline CHLOROPRENE & 114.5 & 5455.2 & 0 & 261675 \\
\hline CHLOROTHALONIL & 170.4 & 5216.0 & 0 & 208162.8 \\
\hline CHLOROTRIFLUOROMETHANE & 6.0 & 286.7 & 0 & 13753 \\
\hline CHLORSULFURON & 0.0 & 0.2 & 0 & 9.39 \\
\hline CHROMIUM & 3912.4 & 38631.4 & 0 & 890005 \\
\hline $\begin{array}{l}\text { CHROMIUM_COMPOUNDS_EXCEPT_CHRO } \\
\text { M }\end{array}$ & 20548.6 & 193615.2 & 0 & 7152107 \\
\hline COBALT & 204.0 & 4950.0 & 0 & 218387 \\
\hline COBALT_COMPOUNDS & 1711.5 & 11994.2 & 0 & 284233.2 \\
\hline COPPER & 6217.4 & 60476.5 & 0 & 1738355 \\
\hline COPPER_COMPOUNDS & 58918.7 & 1262744.0 & 0 & $\begin{array}{c}5164740 \\
2\end{array}$ \\
\hline CREOSOTE & 259.0 & 3386.8 & 0 & 104730 \\
\hline CRESOL_MIXED_ISOMERS_ & 937.2 & 9490.3 & 0 & 232200 \\
\hline CROTONALDEHYDE & 23.7 & 979.2 & 0 & 46305 \\
\hline CUMENE & 380.0 & 6387.3 & 0 & 197016 \\
\hline CUMENE_HYDROPEROXIDE & 38.3 & 1169.5 & 0 & 54463.1 \\
\hline CYANAZINE & 6.5 & 312.7 & 0 & 15001 \\
\hline CYANIDE_COMPOUNDS & 1815.3 & 31339.7 & 0 & 882800 \\
\hline CYCLOHEXANE & 1713.0 & 20121.8 & 0 & 680158 \\
\hline CYCLOHEXANOL & 909.5 & 34606.7 & 0 & 1610800 \\
\hline CYFLUTHRIN & 1.1 & 52.5 & 0 & 2518.95 \\
\hline DAZOMET & 0.6 & 15.2 & 0 & 543.3 \\
\hline DECABROMODIPHENYL_OXIDE & 115.5 & 1375.1 & 0 & 40002 \\
\hline DI_2_ETHYLHEXYL_PHTHALATE & 367.5 & 4970.7 & 0 & 163525 \\
\hline DIALLATE & 0.0 & 0.4 & 0 & 14.82 \\
\hline DIAMINOTOLUENE__MIXED_ISOMERS_ & 2.0 & 87.1 & 0 & 4165 \\
\hline DIAZINON & 9.7 & 463.6 & 0 & 22236.25 \\
\hline DIBENZOFURAN & 5.5 & 130.0 & 0 & 5627.9 \\
\hline DIBROMOTETRAFLUOROETHANE & 0.0 & 2.0 & 0 & 95 \\
\hline DIBUTYL_PHTHALATE & 71.9 & 2249.8 & 0 & 101451 \\
\hline DICAMBA & 59.4 & 2239.0 & 0 & 101661.8 \\
\hline DICHLOROBENZENE_MIXED_ISOMERS_ & 0.7 & 28.4 & 0 & 1356.8 \\
\hline DICHLOROBROMOMETHANE & 1.8 & 85.0 & 0 & 4075 \\
\hline DICHLORODIFLUOROMETHANE & 32.2 & 735.5 & 0 & 25935 \\
\hline DICHLOROFLUOROMETHANE & 0.1 & 5.4 & 0 & 260 \\
\hline DICHLOROMETHANE & 1431.9 & 14343.1 & 0 & 369092 \\
\hline DICHLOROTETRAFLUOROETHANE_CFC_ & 117.1 & 4038.7 & 0 & 184195 \\
\hline DICHLOROTRIFLUOROETHANE & 0.0 & 0.2 & 0 & 9 \\
\hline DICHLORVOS & 0.0 & 0.3 & 0 & 10 \\
\hline DICOFOL & 15.8 & 758.0 & 0 & 36362.24 \\
\hline DICYCLOPENTADIENE & 156.3 & 4138.5 & 0 & 183911.8 \\
\hline
\end{tabular}




\begin{tabular}{|c|c|c|c|c|}
\hline DIEPOXYBUTANE & 0.0 & 0.3 & 0 & 13.58 \\
\hline DIETHANOLAMINE & 495.3 & 7585.4 & 0 & 228941 \\
\hline DIETHYL_SULFATE & 2.6 & 96.3 & 0 & 4515 \\
\hline DIGLYCIDYL_RESORCINOL_ETHER & 0.0 & 0.1 & 0 & 3 \\
\hline DIHYDROSAFROLE & 0.0 & 0.3 & 0 & 13.55 \\
\hline DIISOCYANATES & 1021.7 & 15537.1 & 0 & 562488.1 \\
\hline DIMETHIPIN & 0.1 & 5.2 & 0 & 250 \\
\hline DIMETHOATE & 0.1 & 2.3 & 0 & 109 \\
\hline DIMETHYL_PHTHALATE & 40.5 & 534.9 & 0 & 14698 \\
\hline DIMETHYL_SULFATE & 0.3 & 5.4 & 0 & 167.7 \\
\hline DIMETHYLAMINE & 114.5 & 2286.1 & 0 & 83868 \\
\hline DIMETHYLAMINE_DICAMBA & 0.2 & 6.6 & 0 & 305 \\
\hline DIMETHYLCARBAMYL_CHLORIDE & 0.0 & 0.3 & 0 & 13.57 \\
\hline DINITROBUTYL_PHENOL & 19.9 & 868.3 & 0 & 41429.7 \\
\hline DINITROTOLUENE_MIXED_ISOMERS_ & 49.5 & 1966.5 & 0 & 93632 \\
\hline DIOXIN_AND_DIOXIN_LIKE_COMPOUND & 0.1 & 1.4 & 0 & 56.89069 \\
\hline DIPHENYLAMINE & 15.0 & 442.4 & 0 & 19540 \\
\hline DIPOTASSIUM_ENDOTHALL & 1.5 & 64.0 & 0 & 3028 \\
\hline DIPROPYL_ISOCINCHOMERONATE & 0.0 & 0.2 & 0 & 10 \\
\hline DIURON & 22.5 & 938.7 & 0 & 44744 \\
\hline EPICHLOROHYDRIN & 52.3 & 856.1 & 0 & 29803.1 \\
\hline ETHOPROP & 0.0 & 1.9 & 0 & 90 \\
\hline ETHYL_ACRYLATE & 90.8 & 3156.4 & 0 & 150509.4 \\
\hline ETHYL_CHLOROFORMATE & 0.2 & 5.8 & 0 & 255 \\
\hline ETHYL_DIPROPYLTHIOCARBAMATE & 0.0 & 0.3 & 0 & 12 \\
\hline ETHYLBENZENE & 1629.0 & 16234.4 & 0 & 675617.4 \\
\hline ETHYLENE & 7775.0 & 116900.8 & 0 & 3952796 \\
\hline ETHYLENE_GLYCOL & 4615.8 & 69610.1 & 0 & 2960751 \\
\hline ETHYLENE_OXIDE & 135.7 & 1714.3 & 0 & 42929 \\
\hline ETHYLENE_THIOUREA & 0.5 & 14.2 & 0 & 559 \\
\hline ETHYLENEBISDITHIOCARBAMIC_ACID_ & 0.0 & 1.4 & 0 & 66 \\
\hline ETHYLENEIMINE & 0.0 & 0.2 & 0 & 7.49 \\
\hline ETHYLIDENE_DICHLORIDE & 12.8 & 407.6 & 0 & 14214 \\
\hline FENPROPATHRIN & 0.0 & 0.7 & 0 & 33 \\
\hline FERBAM & 0.0 & 0.2 & 0 & 9.59 \\
\hline FLUOMETURON & 0.4 & 20.0 & 0 & 961 \\
\hline FLUORINE & 20.8 & 409.4 & 0 & 14208 \\
\hline FOLPET & 0.1 & 4.2 & 0 & 201 \\
\hline FOMESAFEN & 95.4 & 4558.2 & 0 & 218648.4 \\
\hline FORMALDEHYDE & 8794.5 & 240336.8 & 0 & $\begin{array}{c}1124903 \\
2\end{array}$ \\
\hline FORMIC_ACID & 6721.0 & 187411.7 & 0 & 7604518 \\
\hline FREON_113 & 199.6 & 6578.4 & 0 & 274832 \\
\hline FURAN & 1.4 & 65.6 & 0 & 3146 \\
\hline GLYCIDOL & 39.7 & 1150.7 & 0 & 42201 \\
\hline
\end{tabular}




\begin{tabular}{|c|c|c|c|c|}
\hline HEPTACHLOR & 0.5 & 21.3 & 0 & 1019.17 \\
\hline HEXACHLORO_1_3_BUTADIENE & 1.5 & 57.1 & 0 & 2617.33 \\
\hline HEXACHLOROBENZENE & 11.3 & 414.6 & 0 & 19709 \\
\hline HEXACHLOROCYCLOPENTADIENE & 0.1 & 6.6 & 0 & 313.6 \\
\hline HEXACHLOROETHANE & 1.3 & 33.1 & 0 & 1151.8 \\
\hline HEXACHLOROPHENE & 0.0 & 0.6 & 0 & 29.53 \\
\hline HEXAZINONE & 0.8 & 34.6 & 0 & 1658 \\
\hline HYDRAZINE & 218.2 & 10439.1 & 0 & 500750.8 \\
\hline HYDRAZINE_SULFATE & 51.2 & 2456.5 & 0 & 117834 \\
\hline HYDROCHLORIC_ACID_1995_AND_AFT & 52993.8 & 266312.6 & 0 & 5986600 \\
\hline HYDROGEN_CYANIDE & 2946.1 & 50569.3 & 0 & 1878928 \\
\hline HYDROGEN_FLUORIDE & 14556.4 & 84458.9 & 0 & 2036184 \\
\hline HYDROGEN_SULFIDE & 11493.0 & 110970.6 & 0 & 4647397 \\
\hline HYDROQUINONE & 610.9 & 22793.6 & 0 & 1083808 \\
\hline IRON_PENTACARBONYL & 18.5 & 885.4 & 0 & 42472 \\
\hline ISOBUTYRALDEHYDE & 63.4 & 1933.7 & 0 & 83540 \\
\hline ISODRIN & 0.0 & 0.0 & 0 & 0.3 \\
\hline ISOPRENE & 70.1 & 1670.1 & 0 & 61593.4 \\
\hline ISOPROPYL_ALCOHOL_MANUFACTURIN & 0.2 & 11.8 & 0 & 567 \\
\hline ISOSAFROLE & 0.0 & 0.4 & 0 & 21.08 \\
\hline LACTOFEN & 0.0 & 0.2 & 0 & 8 \\
\hline LEAD & 4906.8 & 57863.3 & 0 & 2006487 \\
\hline LEAD_COMPOUNDS & 97319.3 & 2329421.0 & 0 & $\begin{array}{c}1.09 \mathrm{E}+0 \\
8\end{array}$ \\
\hline LINDANE & 0.0 & 0.6 & 0 & 27.86 \\
\hline LINURON & 0.2 & 10.6 & 0 & 509 \\
\hline LITHIUM_CARBONATE & 96.8 & 2453.7 & 0 & 85811 \\
\hline M_CRESOL & 73.9 & 3382.6 & 0 & 162201.2 \\
\hline M_DINITROBENZENE & 2.4 & 113.5 & 0 & 5443 \\
\hline M_XYLENE & 114.5 & 2183.5 & 0 & 71000 \\
\hline MALATHION & 3.5 & 158.7 & 0 & 7606.19 \\
\hline MALEIC_ANHYDRIDE & 384.8 & 9892.5 & 0 & 446870.9 \\
\hline MALONONITRILE & 0.0 & 0.3 & 0 & 13.67 \\
\hline MANGANESE & 6682.6 & 99069.2 & 0 & 4362300 \\
\hline MANGANESE_COMPOUNDS & 69236.6 & 456307.2 & 0 & 9559607 \\
\hline MECOPROP & 0.1 & 5.5 & 0 & 265 \\
\hline MERCURY & 31.2 & 426.8 & 0 & 16873.91 \\
\hline MERCURY_COMPOUNDS & 1845.4 & 50968.1 & 0 & 1954509 \\
\hline METHACRYLONITRILE & 289.8 & 13900.2 & 0 & 666777 \\
\hline METHAM_SODIUM & 0.7 & 31.7 & 0 & 1520 \\
\hline METHANOL & 59486.3 & 304890.8 & 0 & 5437770 \\
\hline METHOXONE & 15.2 & 723.0 & 0 & 34683 \\
\hline METHOXONE_SODIUM_SALT & 0.0 & 0.1 & 0 & 4 \\
\hline METHOXYCHLOR & 0.0 & 0.6 & 0 & 30.36 \\
\hline METHYL_ACRYLATE & 41.6 & 693.7 & 0 & 20167 \\
\hline
\end{tabular}




\begin{tabular}{|c|c|c|c|c|}
\hline METHYL_CHLOROCARBONATE & 0.1 & 5.5 & 0 & 260 \\
\hline METHYL_HYDRAZINE & 0.0 & 0.2 & 0 & 8.05 \\
\hline METHYL_IODIDE & 9.5 & 312.2 & 0 & 14295 \\
\hline METHYL_ISOBUTYL_KETONE & 1614.7 & 16465.8 & 0 & 471920 \\
\hline METHYL_ISOCYANATE & 0.0 & 0.2 & 0 & 8.63 \\
\hline METHYL_METHACRYLATE & 1314.1 & 21684.5 & 0 & 886657 \\
\hline METHYL_TERT_BUTYL_ETHER & 824.4 & 32565.4 & 0 & 1550810 \\
\hline METHYLENE_BROMIDE & 3.3 & 84.7 & 0 & 2899 \\
\hline METRIBUZIN & 1.1 & 52.7 & 0 & 2528.77 \\
\hline MIXTURE & 302.7 & 11179.4 & 0 & 532750 \\
\hline MOLYBDENUM_TRIOXIDE & 636.3 & 7827.5 & 0 & 248254.4 \\
\hline MONOCHLOROPENTAFLUOROETHANE & 12.7 & 511.5 & 0 & 24285 \\
\hline MYCLOBUTANIL & 0.0 & 0.1 & 0 & 5 \\
\hline N_BUTYL_ALCOHOL & 4724.0 & 26977.3 & 0 & 451529.6 \\
\hline N_HEXANE & 14654.8 & 88169.6 & 0 & 1950400 \\
\hline N_METHYL_2_PYRROLIDONE & 2939.6 & 52931.5 & 0 & 1572984 \\
\hline N_METHYLOLACRYLAMIDE & 11.2 & 420.9 & 0 & 19958.26 \\
\hline N_NITROSO_N_ETHYLUREA & 0.0 & 0.2 & 0 & 8.59 \\
\hline N_NITROSO_N_METHYLUREA & 0.0 & 0.2 & 0 & 8.56 \\
\hline N_NITROSODI_N_PROPYLAMINE & 0.0 & 0.0 & 0 & 1 \\
\hline N_NITROSODIPHENYLAMINE & 0.0 & 0.2 & 0 & 10 \\
\hline N_NITROSOMETHYLVINYLAMINE & 0.0 & 0.2 & 0 & 7.49 \\
\hline N_NITROSOPIPERIDINE & 0.0 & 0.4 & 0 & 21.07 \\
\hline N_N_DIMETHYLANILINE & 0.9 & 29.6 & 0 & 1258 \\
\hline N_N_DIMETHYLFORMAMIDE & 437.2 & 12857.3 & 0 & 600490 \\
\hline NABAM & 3.3 & 157.2 & 0 & 7540 \\
\hline NALED & 0.0 & 0.2 & 0 & 10 \\
\hline NAPHTHALENE & 922.6 & 7168.1 & 0 & 147697 \\
\hline NICKEL & 2510.0 & 36824.5 & 0 & 1654067 \\
\hline NICKEL_COMPOUNDS & 12382.3 & 172867.0 & 0 & 6909875 \\
\hline NICOTINE_AND_SALTS & 316.9 & 4351.0 & 0 & 120836 \\
\hline NITRAPYRIN & 0.0 & 0.5 & 0 & 21.603 \\
\hline NITRATE_COMPOUNDS & $\begin{array}{c}114715 \\
1\end{array}$ & 722307.2 & 0 & $\begin{array}{c}2137602 \\
5\end{array}$ \\
\hline NITRIC_ACID & 7944.2 & 162499.5 & 0 & 6303631 \\
\hline NITRILOTRIACETIC_ACID & 18.4 & 597.6 & 0 & 22363 \\
\hline NITROBENZENE & 104.4 & 3340.6 & 0 & 146196 \\
\hline NITROGLYCERIN & 107.9 & 3060.8 & 0 & 118369 \\
\hline NITROMETHANE & 28.7 & 1167.8 & 0 & 55523 \\
\hline O_ANISIDINE & 0.1 & 5.0 & 0 & 242 \\
\hline O_CRESOL & 18.6 & 689.5 & 0 & 32015 \\
\hline O_DINITROBENZENE & 2.3 & 108.7 & 0 & 5212 \\
\hline O_TOLUIDINE & 3.0 & 88.0 & 0 & 3461 \\
\hline O_TOLUIDINE_HYDROCHLORIDE & 0.0 & 0.5 & 0 & 21.68 \\
\hline O_XYLENE & 99.0 & 1818.5 & 0 & 73000 \\
\hline
\end{tabular}




\begin{tabular}{|c|c|c|c|c|}
\hline OCTACHLOROSTYRENE & 0.2 & 6.6 & 0 & 315.92 \\
\hline OSMIUM_TETROXIDE & 0.0 & 0.2 & 0 & 7.36 \\
\hline OXYDIAZON & 0.1 & 5.3 & 0 & 250 \\
\hline OXYFLUORFEN & 0.0 & 1.4 & 0 & 64.77 \\
\hline OZONE & 301.5 & 4976.7 & 0 & 141280.2 \\
\hline P_CHLOROANILINE & 0.6 & 30.2 & 0 & 1450 \\
\hline P_CRESIDINE & 0.2 & 6.9 & 0 & 260 \\
\hline P_CRESOL & 38.1 & 1730.4 & 0 & 82960.48 \\
\hline P_DINITROBENZENE & 0.6 & 27.1 & 0 & 1298 \\
\hline P_NITROANILINE & 0.0 & 0.4 & 0 & 20.7 \\
\hline P_PHENYLENEDIAMINE & 8.9 & 311.7 & 0 & 14250 \\
\hline P_XYLENE & 294.2 & 6244.9 & 0 & 237000 \\
\hline PARALDEHYDE & 0.1 & 1.3 & 0 & 45.2 \\
\hline PARAQUAT_DICHLORIDE & 14.2 & 488.0 & 0 & 22573 \\
\hline PENDIMETHALIN & 27.3 & 1252.8 & 0 & 60080 \\
\hline PENTACHLOROBENZENE & 0.3 & 10.0 & 0 & 459.41 \\
\hline PENTACHLOROETHANE & 0.7 & 29.5 & 0 & 1411 \\
\hline PENTACHLOROPHENOL & 2.6 & 70.9 & 0 & 2858.5 \\
\hline PENTOBARBITAL_SODIUM & 0.0 & 0.1 & 0 & 3 \\
\hline PERACETIC_ACID & 19.6 & 464.3 & 0 & 20691 \\
\hline PERMETHRIN & 2.4 & 115.3 & 0 & 5532 \\
\hline PHENANTHRENE & 76.0 & 1963.9 & 0 & 89048.2 \\
\hline PHENOL & 3253.0 & 22994.2 & 0 & 569862 \\
\hline PHENOTHRIN & 0.0 & 0.4 & 0 & 20 \\
\hline PHENYTOIN & 0.5 & 25.0 & 0 & 1200 \\
\hline PHOSGENE & 6.2 & 175.1 & 0 & 7600 \\
\hline PHOSPHINE & 2.4 & 102.2 & 0 & 4845.3 \\
\hline PHOSPHORUS_YELLOW_OR_WHITE_ & 23.1 & 536.9 & 0 & 18399.55 \\
\hline PHTHALIC_ANHYDRIDE & 102.6 & 1889.0 & 0 & 68264 \\
\hline PICLORAM & 0.1 & 4.4 & 0 & 204 \\
\hline PICRIC_ACID & 29.9 & 1427.7 & 0 & 68483 \\
\hline PIPERONYL_BUTOXIDE & 0.0 & 0.3 & 0 & 15 \\
\hline POLYCHLORINATED_ALKANES & 0.2 & 6.8 & 0 & 319 \\
\hline POLYCHLORINATED_BIPHENYLS & 1988.0 & 37594.1 & 0 & 1104719 \\
\hline POLYCYCLIC_AROMATIC_COMPOUNDS & 938.2 & 14545.6 & 0 & 541907.9 \\
\hline POTASSIUM_BROMATE & 0.1 & 5.2 & 0 & 250 \\
\hline POTASSIUM_DIMETHYLDITHIOCARBAMA & 2.2 & 107.8 & 0 & 5173 \\
\hline POTASSIUM_N_METHYLDITHIOCARBAMA & 17.4 & 831.0 & 0 & 39862 \\
\hline PROMETRYN & 1.1 & 38.7 & 0 & 1455 \\
\hline PRONAMIDE & 0.0 & 0.4 & 0 & 16.03 \\
\hline PROPACHLOR & 0.0 & 0.1 & 0 & 4.01 \\
\hline PROPANE_SULTONE & 0.1 & 6.9 & 0 & 333.03 \\
\hline PROPANIL & 14.9 & 498.7 & 0 & 20500 \\
\hline PROPARGITE & 0.0 & 2.3 & 0 & 110 \\
\hline
\end{tabular}




\begin{tabular}{|c|c|c|c|c|}
\hline PROPARGYL_ALCOHOL & 0.6 & 18.5 & 0 & 844 \\
\hline PROPICONAZOLE & 96.4 & 4347.3 & 0 & 208160.9 \\
\hline PROPIONALDEHYDE & 94.9 & 1714.9 & 0 & 54668 \\
\hline PROPYLENE & 3644.0 & 44834.8 & 0 & 1519031 \\
\hline PROPYLENE_OXIDE & 167.7 & 2730.8 & 0 & 80153 \\
\hline PROPYLENEIMINE & 0.1 & 5.1 & 0 & 228.4 \\
\hline PYRIDINE & 327.0 & 5927.0 & 0 & 160005 \\
\hline QUINOLINE & 7.0 & 311.3 & 0 & 14924 \\
\hline QUINONE & 0.2 & 7.5 & 0 & 357 \\
\hline QUINTOZENE & 0.2 & 5.6 & 0 & 247 \\
\hline S_S_S_TRIBUTYLTRITHIOPHOSPHATE & 0.1 & 3.8 & 0 & 180 \\
\hline SACCHARIN_MANUFACTURING_NO_SU & 0.0 & 0.0 & 0 & 0.97 \\
\hline SAFROLE & 0.0 & 0.5 & 0 & 21.06 \\
\hline SEC_BUTYL_ALCOHOL & 213.1 & 2929.1 & 0 & 100890.6 \\
\hline SELENIUM & 122.4 & 2722.7 & 0 & 109136 \\
\hline SELENIUM_COMPOUNDS & 794.7 & 6650.8 & 0 & 155841 \\
\hline SILVER & 125.7 & 2895.3 & 0 & 118216 \\
\hline SILVER_COMPOUNDS & 105.0 & 1652.3 & 0 & 45311 \\
\hline SIMAZINE & 34.6 & 1167.4 & 0 & 40202 \\
\hline SODIUM_AZIDE & 1.1 & 52.5 & 0 & 2520.44 \\
\hline SODIUM_DICAMBA & 4.5 & 157.4 & 0 & 6350 \\
\hline SODIUM_DIMETHYLDITHIOCARBAMATE & 87.1 & 2434.9 & 0 & 100677.5 \\
\hline SODIUM_FLUOROACETATE & 0.0 & 0.3 & 0 & 12.62 \\
\hline SODIUM_NITRITE & 4104.2 & 107186.1 & 0 & 4800500 \\
\hline STRYCHNINE_AND_SALTS & 0.0 & 0.1 & 0 & 5 \\
\hline STYRENE & 11876.8 & 49754.2 & 0 & 1090671 \\
\hline STYRENE_OXIDE & 0.0 & 0.7 & 0 & 33.97 \\
\hline SULFURIC_ACID_1994_AND_AFTER_A & 43005.9 & 252987.4 & 0 & 4477500 \\
\hline SULFURYL_FLUORIDE & 194.8 & 2407.8 & 0 & 51275 \\
\hline TERT_BUTYL_ALCOHOL & 708.8 & 24657.4 & 0 & 1170380 \\
\hline TETRABROMOBISPHENOL_A & 55.4 & 1783.1 & 0 & 68882 \\
\hline TETRACHLOROETHYLENE & 438.7 & 5053.5 & 0 & 184062 \\
\hline TETRACHLORVINPHOS & 0.1 & 5.3 & 0 & 255 \\
\hline TETRACYCLINE_HYDROCHLORIDE & 0.8 & 39.8 & 0 & 1908 \\
\hline TETRAFLUOROETHYLENE & 142.5 & 4504.3 & 0 & 199390.4 \\
\hline THALLIUM & 47.0 & 2252.3 & 0 & 108038 \\
\hline THALLIUM_COMPOUNDS & 535.9 & 5886.1 & 0 & 128103 \\
\hline THIABENDAZOLE & 45.7 & 2179.0 & 0 & 104520.8 \\
\hline THIOACETAMIDE & 0.0 & 0.6 & 0 & 29.44 \\
\hline THIOBENCARB & 0.0 & 0.2 & 0 & 10 \\
\hline THIODICARB & 0.5 & 24.3 & 0 & 1165 \\
\hline THIOPHANATE_METHYL & 0.6 & 28.4 & 0 & 1361 \\
\hline THIOSEMICARBAZIDE & 0.0 & 0.2 & 0 & 7.49 \\
\hline THIOUREA & 1.9 & 86.7 & 0 & 4158 \\
\hline
\end{tabular}




\begin{tabular}{|c|c|c|c|c|}
\hline THIRAM & 22.9 & 433.4 & 0 & 18788 \\
\hline TITANIUM_TETRACHLORIDE & 14.1 & 365.9 & 0 & 15005 \\
\hline TOLUENE & 11914.7 & 67310.6 & 0 & 2075190 \\
\hline TOLUENE_2_4_DIISOCYANATE & 1.1 & 24.6 & 0 & 940 \\
\hline TOLUENE_2_6_DIISOCYANATE & 0.2 & 7.2 & 0 & 250.2 \\
\hline TOLUENE_DIISOCYANATE_MIXED_ISO & 21.5 & 272.8 & 0 & 8677.83 \\
\hline TOXAPHENE & 0.1 & 2.8 & 0 & 131.503 \\
\hline TRADE_SECRET_CHEMICAL & 0.7 & 35.3 & 0 & 1695 \\
\hline TRANS_1_3_DICHLOROPROPENE & 0.2 & 9.1 & 0 & 430 \\
\hline TRANS_1_4_DICHLORO_2_BUTENE & 0.0 & 0.3 & 0 & 13.55 \\
\hline TRIADIMEFON & 0.0 & 0.2 & 0 & 9 \\
\hline TRIALLATE & 0.0 & 0.5 & 0 & 24 \\
\hline TRIBENURON_METHYL & 0.0 & 0.2 & 0 & 9.27 \\
\hline TRICHLORFON & 0.0 & 0.1 & 0 & 5 \\
\hline TRICHLOROACETYL_CHLORIDE & 0.0 & 0.2 & 0 & 7.2 \\
\hline TRICHLOROETHYLENE & 1055.1 & 10365.2 & 0 & 285471.5 \\
\hline TRICHLOROFLUOROMETHANE & 68.7 & 2063.7 & 0 & 94578 \\
\hline TRICLOPYR_TRIETHYLAMMONIUM_SALT & 0.0 & 0.0 & 0 & 1 \\
\hline TRIETHYLAMINE & 165.6 & 2298.0 & 0 & 62706 \\
\hline TRIFLURALIN & 2.8 & 83.8 & 0 & 3099.45 \\
\hline TRIPHENYLTIN_HYDROXIDE & 0.9 & 29.4 & 0 & 1278 \\
\hline TRYPAN_BLUE & 0.0 & 0.6 & 0 & 27.63 \\
\hline URETHANE & 9.7 & 427.0 & 0 & 20440.8 \\
\hline VANADIUM_EXCEPT_WHEN_CONTAINED & 263.6 & 4998.9 & 0 & 166427 \\
\hline VANADIUM_COMPOUNDS & 13417.0 & 74032.2 & 0 & 2025898 \\
\hline VINYL_ACETATE & 788.0 & 14326.5 & 0 & 613894.9 \\
\hline VINYL_CHLORIDE & 227.7 & 3320.1 & 0 & 92047 \\
\hline VINYL_FLUORIDE & 31.1 & 923.8 & 0 & 33176 \\
\hline VINYLIDENE_CHLORIDE & 16.0 & 400.4 & 0 & 15552 \\
\hline WARFARIN_AND_SALTS & 0.0 & 0.2 & 0 & 7.6 \\
\hline XYLENE_MIXED_ISOMERS_ & 7189.9 & 34767.4 & 0 & 907146.1 \\
\hline ZINC_FUME_OR_DUST_ & 3603.6 & 111308.4 & 0 & 5291548 \\
\hline ZINC_COMPOUNDS & $\begin{array}{c}111384 \\
3\end{array}$ & 890554.2 & 0 & $\begin{array}{c}2264803 \\
4\end{array}$ \\
\hline
\end{tabular}




\section{CURRICULUM VITAE}

Katlyn E McGraw

April 2021

\section{PERSONAL STATEMENT}

'A dedicated Environmental Health Science PhD candidate with a thorough background in analytical chemistry, human subjects research, air monitoring, and environmental epidemiology.'

As an environmental health scientist, I am interested in conducting actionable research to address current environmental issues, influence state and local policy, and engage communities in participatory research to enact necessary change. My personal goals are to gain further expertise in actionable research to increase pollution regulation. In addition, I would like to improve upon existing exposure assessment and statistical methods, and to engage more with affected communities and stakeholders. My future career goal is to gain a leadership research position in environmental health to reduce pollution. Currently, I hold two leadership positions, one as a Superfund Research Program Trainee Leader, and another as American Public Health Association Environment Section Councilor. I have eight publications, one manuscript recently published in Environmental Research, and two manuscripts in progress.

\section{EDUCATION}

University of Louisville, Louisville, KY, PhD 2021. Environmental Health Science University of Louisville, Louisville, KY, MPH 2016. Environmental Health Science University of Louisville, Louisville, KY, BA 2011. Major: Chemistry, Minor: Spanish

\section{RELEVANT EXPERIENCE}

2016-2021 PhD Candidate

University of Louisville, Louisville, KY

Mentor: Aruni Bhatnagar

The major focus of my dissertation is to understand how gaseous pollutants such as volatile organic compounds contribute to cardiovascular disease. The first aim examines associations between cardiovascular risk factors and urinary biomarkers of exposure. The second aim examines ecological associations between toxic releases and cardiovascular mortality. The third aim analyzes multiple urinary biomarkers of exposure as components of mixtures and how these mixtures contribute to cardiovascular disease.

2016-2021 Graduate Research Assistant University of Louisville, Louisville, KY Mentor: Aruni Bhatnagar 
2018-2021

2013-2014

2011-2012
Reviewed contributors to cardiovascular disease: greenness, built environment, and exposure to volatile organic compounds; conducted passive and active air sampling of nitrogen oxides, ultra-fine particulates, and particulate matter; planned and recruited participants in the HEAL study; and analyzed cohort data for epidemiological associations of biomarkers of exposure and cardiovascular disease.

\section{Independent Contractor}

Environmental Defense Fund, Houston, TX

Mentor: Elena Craft

Quantified industrial emissions after Hurricane Harvey; created an action plan for elevated concentrations of benzene in air; supervised Houston city air monitoring pilot program; assisted community engagement; reviewed literature for low benzene exposure and immunological and hematological disorders; visualized data for industrial disaster response.

\section{Clinical Research Consultant}

ThreeWire, Louisville, KY

Mentor: Brett Weaver

Data collection for phase II randomized clinical trial with Pfizer; mined data from electronic medical records; recruited participants; conducted participant interviews.

\section{Clinical Research Associate}

Norton Leatherman Spine Center, Louisville KY

Mentor: Leah Carreon

Collected data and recruited participants for multiple clinical trials and outcomes research following spine surgeries; managed National Neurosurgery Quality Outcomes Database; examined patients for scoliosis; used common instruments Short Form 36 (SF36) and the Euroqol EQ5D to measure patients' quality of life and disability index.

\section{Production Technician III}

Siemens PETNET Solutions, Louisville, KY

Mentor: Virginia Coakley

Performed quality assurance and control of diagnostic radiopharmaceuticals, maintained and operated particle accelerator, chemistry box, and gas chromatography instruments; dispensed and delivered radiopharmaceuticals; formulated new research radiopharmaceuticals.

\section{Fuels Technician}

Safety Kleen, Smithfield, KY

Mentor: Keith Smiley

Characterized waste streams for fuel recycling; maintained and operated multiple gas chromatography instruments, inductively coupled plasma instrument, and digestion ovens; analyzed chromatograms for polychlorinated biphenyls and metal concentrations; performed basic wet laboratory techniques such as $\mathrm{pH}$, flammability, viscosity.

\section{Summer Research Assistant}

Universidad de La Plata CONICET, La Plata, Argentina

Mentor: Francisco Ibanez

Synthesized and characterized gold nanoparticles; analyzed gold nanoparticles for plasmon resonance in the use of solar cells; performed organic chemistry technique such as distillation. 
Supervised and managed retail pharmacy duties; compounded pharmaceuticals; translated instructions for Spanish speakers; customer service; extensive pharmaceutical knowledge.

\section{SKILLS}

SAS · R · Data Analysis · Mapping · Fluent Spanish Speaker · Bench Laboratory · Laboratory Instrumentation · Exposure Assessment · Air Monitoring

\section{PUBLICATIONS}

McGraw, K.E, Rai SN, Riggs DW, and Bhatnagar A. Changes in County Level Toxic Releases are Associated with Increased Circulatory Disease Mortality between 2002-2012. In Preparation.

McGraw, K.E, Konkle SL, Riggs DW, Rai SN, Keith RJ, Amraotkar A, Oshunbade A, Hall M, Bhatnagar A. Volatile Organic Compound Metabolites are Sensitive Markers of Smoking Patterns and Status in African Americans: the Jackson Heart Study. In Preparation.

McGraw, K. E., et al. (2021). "Exposure to Volatile Organic Compounds - Acrolein, 1,3Butadiene, and Crotonaldehyde - is Associated with Vascular Dysfunction." Environmental Research: 110903.

Phillips A, Clark-Leach G, McGraw, K.E, Quist A, Bernhardt C, Keldermen K. Preparing for the Next Storm, Learning from the Man-Made Environmental Disasters that Followed Hurricane Harvey. Environmental Integrity Project. 16 August 2018.

Jazini, E., Gum, J., Glassman, S., Crawford III, C.H., Djurasovic, M., Owens II, R.K., Dimar II, J.R., McGraw, K.E., Carreon, L.Y. Cost-Effectiveness of Circumferential Fusion for Lumbar Spondylolisthesis: Propensity-Matched Comparison of Transforaminal Lumbar Interbody Fusion with Anterior-Posterior Fusion. Spine Journal, 2018.

Mladen Djurasovic, MD, McGraw, K.E, Kelly Bratcher, RN, CCRP, Charles H. Crawford III, MD, John R. Dimar II, MD, Rolando M. Puno, MD, Steven D. Glassman, MD, R. Kirk Owens II, MD, Leah Y. Carreon, MD, MSc. Randomized Trial of Cell Saver in Two-to Three- Level Lumbar Instrumented Posterior Fusions. J Neurosurg Spine, 2018.

Laratta JL, Bratcher KR, McGraw K.E., Carreon LY, Owens RK $2^{\text {nd }}$, Outcomes and revision rates Following multilevel anterior cervical discectomy and fusion. Journal of Spine Surgery,2018.

Gvozdyev, B.V., Carreon, L.Y., Graves, C.M., Riley, S.A., McGraw, K.E., Head, R.J., Dimar II, J.R., Glassman, S.D. Patient-reported outcome scores underestimate the impact of major complications in patients undergoing spine surgery for degenerative conditions. J Neurosurg Spine, 2017. 27(4): p. 397-402.

Owens II, R.K., Djurasovic, M., Onyekwelu, I., Bratcher, K.R., McGraw, K.E., Carreon, L.Y. Outcomes and revision rates in normal, overweight, and obese patients 5 years after lumbar fusion. Spine J, 2016. 16(10): p. 1178-1183.

Carreon, Djurasovic, M., Dimar II, J.R., Owens II, R.K., Crawford III, C.H., Puno, R.M., Bratcher, K.R., McGraw, K.E., Glassman, S.D. Can the anxiety domain of EQ-5D and mental health items 
from SF-36 help predict outcomes after surgery for lumbar degenerative disorders? J Neurosurg Spine, 2016. 25(3): p. 352-6.

Carreon, L.Y., Owens II, R.K., Bratcher, K.R., McGraw, K.E. Outcomes and Revision Rates following Multilevel Anterior Cervical Discectomy and Fusion. The Spine Journal, 2015. 15(10): p. S226-S227.

\section{ABSTRACTS}

Changes in County Level Toxic Releases are Associated with Increased Circulatory Disease Mortality between 2002-2012. Katlyn E. McGraw, Shesh N. Rai, Daniel W. Riggs, and Aruni Bhatnagar. Superfund Research Program Annual Conference. College Station, TX. December 2020.

Exposure to Volatile Organic Compounds - Acrolein, 1,3-Butadiene, and Crotonaldehyde - is Associated with Vascular Dysfunction in the Louisville Healthy Heart Study. McGraw KE; Riggs DW; Rai SN; Navas-Acien A; Xie Z; Lorkiewicz P; Lynch JF; Zafar N; Krishnasamy S; Taylor KC; Conklin DJ; DeFilippis AP; Srivastava S; Aruni Bhatnagar. International Society of

Environmental Epidemiology, Washington D.C., August 2020.

'Toxic Release Sites Cluster in Space in Jefferson County, Kentucky.' McGraw KE, Srivastava S, Bhatnagar A. Superfund Research Program Annual Conference, Seattle, WA, November 2019.

'Toxic Release Sites Cluster in Space in Jefferson County, Kentucky.' McGraw KE, Srivastava S, Bhatnagar A. Research!Louisville, Louisville, KY, September 2019.

'Association of Volatile Organic Compound Exposure and Catecholamines.' McGraw KE, Riggs DW, Xie Z, Lorkiewicz P, Krivokhizhina T, Yeager R, Conklin DJ, DeFillipis A, Srivastava S, Bhatnagar A. Society of Toxicology, Baltimore, MD, March 2019.

'Association of Volatile Organic Compound Exposure and Catecholamines.' McGraw KE, Riggs DW, Xie Z, Lorkiewicz P, Krivokhizhina T, Yeager R, Conklin DJ, DeFillipis A, Srivastava S, Bhatnagar A. Superfund Research Conference, Sacramento, CA, November 2018.

'Association of Volatile Organic Compound Exposure and Catecholamines.' McGraw KE, Riggs DW, Xie Z, Lorkiewicz P, Krivokhizhina T, Yeager R, Conklin DJ, DeFillipis A, Srivastava S, Bhatnagar A. Research!Louisville, Louisville, KY, October 2018.

'Exposure to Environmental Volatile Organic Chemicals is Associated with Cardiovascular Risk.' McGraw KE, Riggs DW, Xie Z, Lorkiwicz P, Krivokhizhina T, Yeager R, Conklin DJ, Defillipis A, Srivastava S, Bhatnagar A. Annual Kentucky Public Health Association Conference, Cincinnati, OH, April 2018.

'Exposure to Environmental Volatile Organic Chemicals is Associated with Cardiovascular Risk.' McGraw KE, Riggs DW, Xie Z, Lorkiewicz P, Krivokhizhina T, Yeager R, Conklin DJ, DeFillipis A, Srivastava S, Bhatnagar A. Superfund Research Conference, Philadelphia, PA, December 2017.

'Association between Exposure to Volatile Organic Chemicals and Cardiovascular Injury.' McGraw KE, Srivastava S, Zafar N, Bhatnagar A. Research!Louisville, Louisville, KY, September 2017. 
'Global Health Initiatives: Addressing the Knowledge Gap Regarding Over the Counter Medication Among Newly Arriving Refugees Resettling in the Louisville Area.' McGraw KE, Wilson R, Ford R, Bosson R, Peyrani P, Carrico R. Research! Louisville, Louisville KY, September 2015.

'Global Health Initiatives: Addressing the Knowledge Gap Regarding Over the Counter Medication Among Newly Arriving Refugees Resettling in the Louisville Area.' McGraw KE, Wilson R, Ford R, Bosson R, Peyrani P, Carrico R. Annual Kentucky Public Health Association, Owensboro, KY, April 2014.

\section{PRESENTATIONS}

'Applications of Environmental Health.' University of Louisville, Invited Guest Speaker, PHEH 301 Public Health and Our Environment, Louisville, KY, September 2019.

'Exposure to Environmental Chemicals is Associated with Cardiovascular Disease Risk.' University of North Carolina Chapel Hill, Invited Guest Speaker, Environmental Epidemiology Seminar, Chapel Hill, NC, January 2019.

\section{REVIEW ACTIVITIES}

Abstract reviewer for APHA Annual Conference November 2021. Reviewed six abstracts for the Environmental Section.

Abstract reviewer for APHA Annual Conference November 2020. Reviewed six abstracts for the Environmental Section.

Abstract reviewer for APHA Annual Conference November 2019. Reviewed six abstracts for the Environmental Section.

Abstract reviewer for APHA Annual Conference November 2018. Reviewed twelve abstracts for the Environmental Section.

\section{CERTIFICATES}

2020 Mediation, Moderation, and Conditional Process Analysis, Statistical Horizons

2020 Transdisciplinary Environmental Science for Society (TESS), University of Arizona

2019 GIS Workshop, Columbia University, New York, NY

2019 Publishing Academy, University of Louisville, Louisville, KY

2018 Disaster Research Training Workshop, Texas A\&M TEEX, College Station, TX

\section{PROFESSIONAL MEMBERSHIP}

ISEE International Society of Environmental Epidemiology

SOT Society of Toxicology

SETAC Society of Environmental Toxicology 
APHA American Public Health Association

KPHA Kentucky Public Health Association

\section{LEADERSHIP}

2020-2023

2019-2020

2019-2020

2016-2017

APHA Environment Section Councilor

SRP Trainee Leader

Graduate Student Council EOHS Department Representative

Student Government Association SPHIS Secretary

\section{SERVICE}

2019-2020

2018-2019

Earth Walk Organizer

2017-2020

APHA Chemicals and Health Committee

2010-2011

West Jefferson Cunty Community Task Force Board Member

Adelante Hispanic Achievers Tutor

\section{AWARDS}

2021

2020

\section{Dean's Citation Award}

KC Donnelly Award

\section{REFERENCES}

Aruni Bhatnagar

PhD

aruni.bhatnagar@louisville.edu

University of Louisville

Leah Carreon

leah.carreon@nortonhealthcare.org

MD, MSc

Norton Healthcare

Elena Craft

Head Scientist

ecraft@edf.org

Environmental Defense Fund 